\title{
Diabetes Group Medical Visits and Biophysical Outcomes of Care in Uninsured Persons with Diabetes
}

Jennifer A. Mallow

West Virginia University

Follow this and additional works at: https://researchrepository.wvu.edu/etd

\section{Recommended Citation}

Mallow, Jennifer A., "Diabetes Group Medical Visits and Biophysical Outcomes of Care in Uninsured Persons with Diabetes" (2011). Graduate Theses, Dissertations, and Problem Reports. 3403.

https://researchrepository.wvu.edu/etd/3403

This Dissertation is protected by copyright and/or related rights. It has been brought to you by the The Research Repository @ WVU with permission from the rights-holder(s). You are free to use this Dissertation in any way that is permitted by the copyright and related rights legislation that applies to your use. For other uses you must obtain permission from the rights-holder(s) directly, unless additional rights are indicated by a Creative Commons license in the record and/ or on the work itself. This Dissertation has been accepted for inclusion in WVU Graduate Theses, Dissertations, and Problem Reports collection by an authorized administrator of The Research Repository @ WVU.

For more information, please contact researchrepository@mail.wvu.edu. 
Diabetes Group Medical Visits and Biophysical Outcomes of Care in Uninsured Persons with Diabetes

\author{
Jennifer A. Mallow
}

Dissertation submitted to the School of Nursing at West Virginia University in partial fulfillment of the requirements for the degree of Doctor of Philosophy in Nursing

\author{
Cynthia A. Persily PhD, RN, FAAN, Chair \\ Stacey Culp, PhD \\ Georgia Narsavage, PhD, CRNP \\ Heidi Putman-Casdorph, DSN, RN \\ Laurie Theeke, PhD, C-FNP \\ Tara Whetzel, PharmD
}

\author{
School of Nursing \\ Morgantown, West Virginia
}

2011

Key Words: Diabetes, Group Medical Visits, Quality Health Outcomes Model, Uninsured, Rural Populations 


\begin{abstract}
Diabetes Group Medical Visits and Biophysical Outcomes of Care in Uninsured Persons with Diabetes

Jennifer A Mallow
\end{abstract}

Background: Rural populations with low socioeconomic status are at higher risk of late diabetes diagnosis, poor diabetes control, decreased self-management, and development of complications. Diabetes Group Medical Visits (DGMVs) have been used to improve outcomes for persons with diabetes. A gap in the literature exists related to the effectiveness of DGMVs for uninsured persons with diabetes.

Aims: The aims of the study were to describe the characteristics of uninsured persons with diabetes cared for in a free clinic, describe the biophysical outcomes of care, explore the differences in biophysical outcomes of care before and after attending DGMVs versus receiving usual care, and explore the impact of dose of DGMVs on biophysical outcomes of care.

Methods: This retrospective study was conducted at a free clinic in West Virginia, using chart review of a convenience sample of patients following approval of the WVU IRB. The inclusion criteria were: 1) age $\geq 18$ years, 2) diagnosis of diabetes, 3) uninsured and received care at a free clinic between May 2007 and August 18, 2009. A total of 111 patients were studied. There were 53 participants who attended DGMVs and 58 participants who received usual care. Statistical Package of Social Sciences (SPSS), version 18 was used for analysis of the data.

Results: The majority of the patients were female, white, severely obese, had a high-school education or less, were age 50 or younger, had a mean of 5 co-morbid conditions other than diabetes, and drove long distances to receive care. The patients who attended DGMVs had a higher $\mathrm{HgA} 1 \mathrm{C}$, reported more pain, had increased depression levels and were more obese at baseline than those who received usual care. There was a statistically significant decrease in 
systolic blood pressure from time one to time two in patients who attended DGMVs. There was no significant impact on biophysical outcomes of care in patients who received usual care from time one to time two. Dose of DGMVs did not impact biophysical outcomes of care.

Conclusion: DGMV as an intervention is not enough to improve biophysical outcomes in this population. Interventions targeted to the unique characteristics of this population are needed to prevent devastating complications. 


\section{TABLE OF CONTENTS}

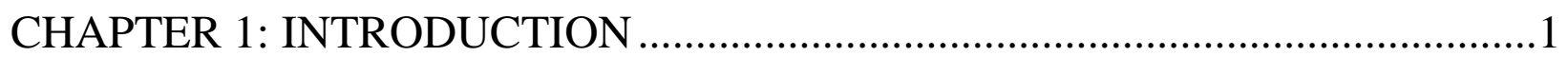

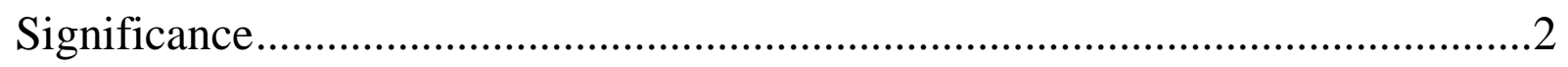

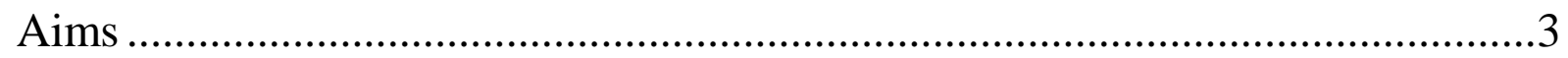

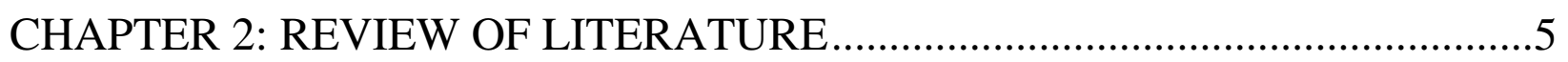

Conceptual Framework: Quality Health Outcomes Model ...................................5

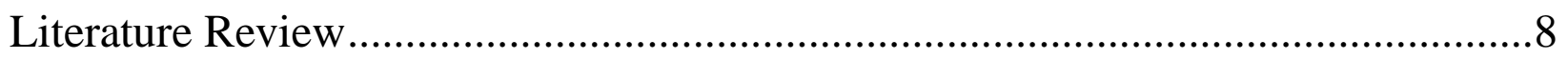

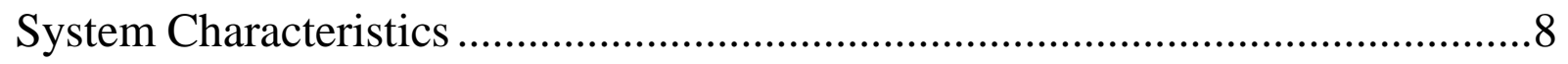

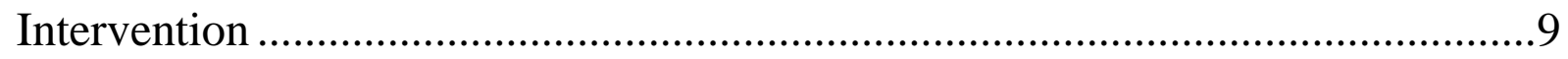

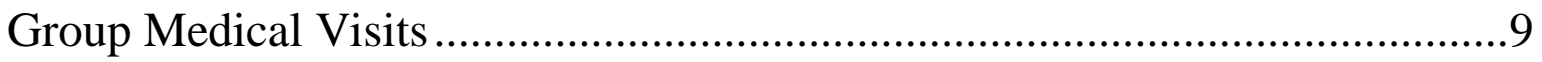

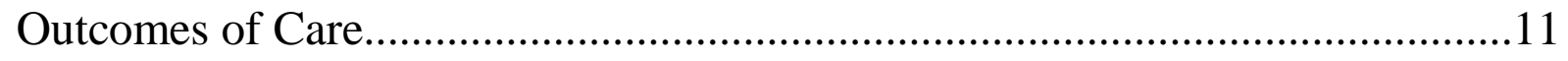

Biophysical Outcomes of Care ….............................................................12

Biophysical Outcomes \& Diabetes Group Medical Visits as an Intervention 13

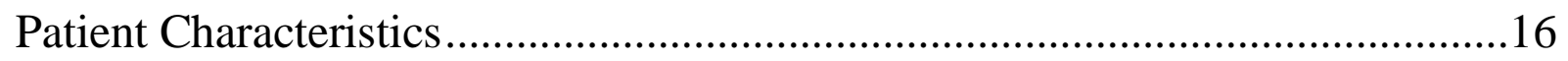

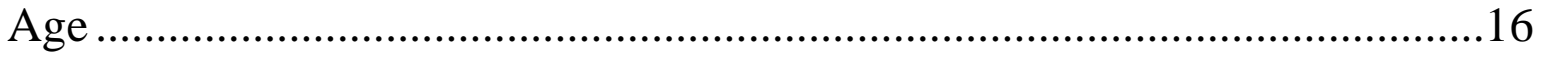

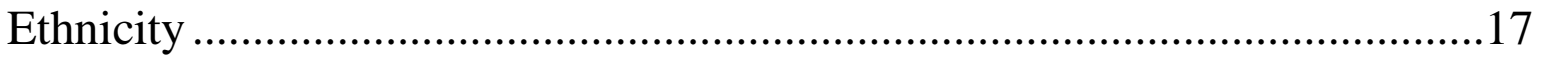

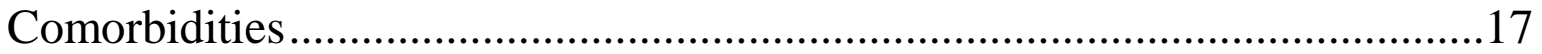


Other Patient Characteristics......................................................................18

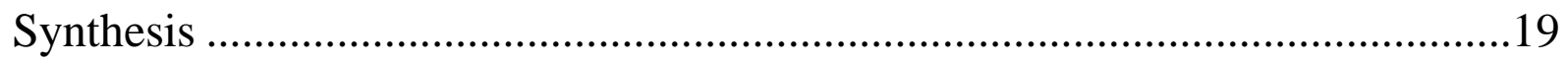

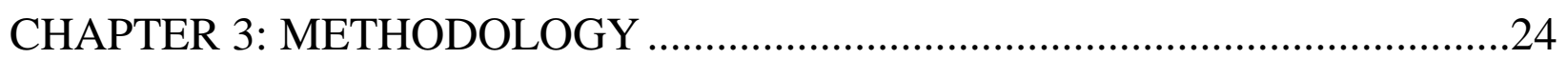

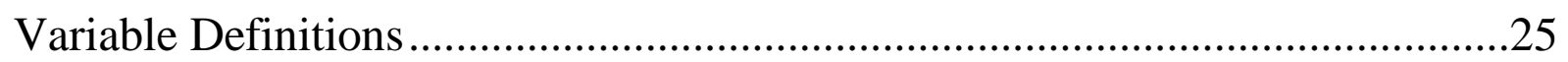

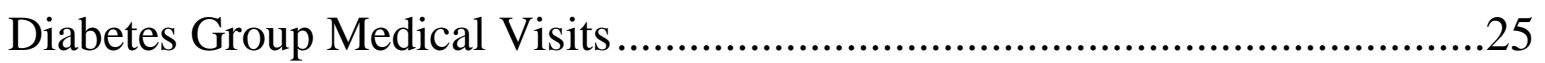

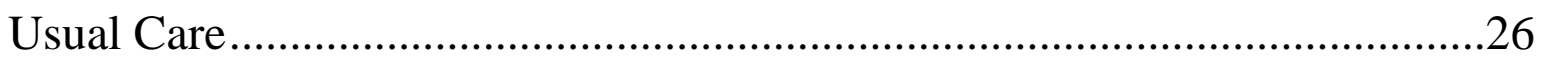

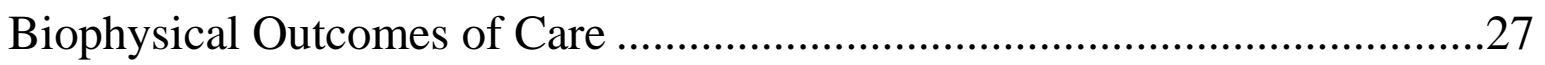

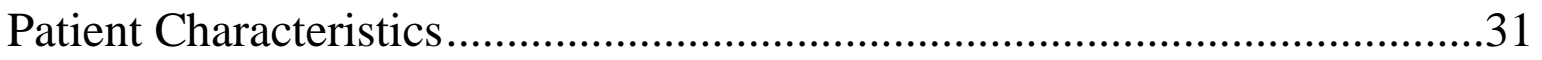

Sample

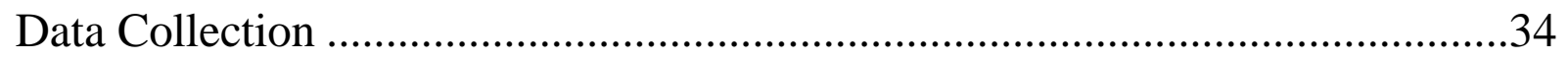

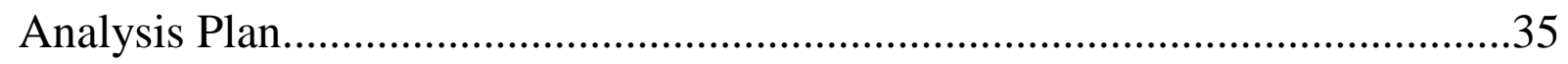

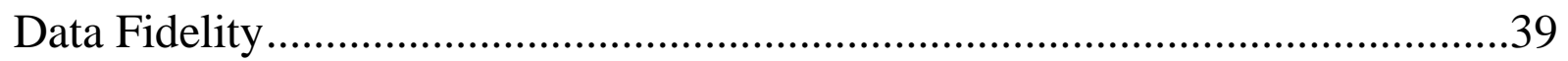

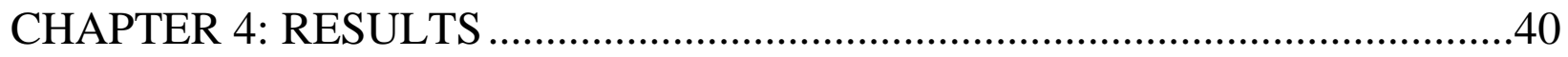

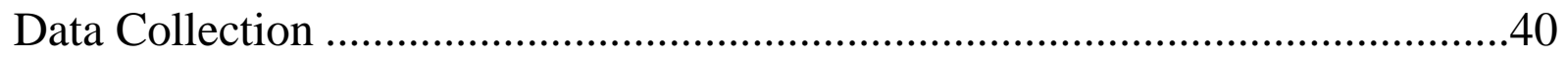

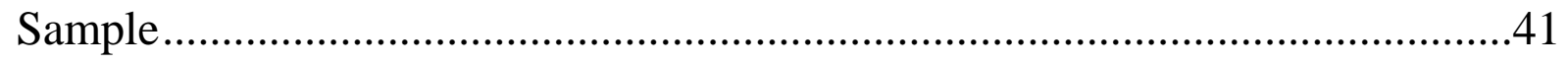

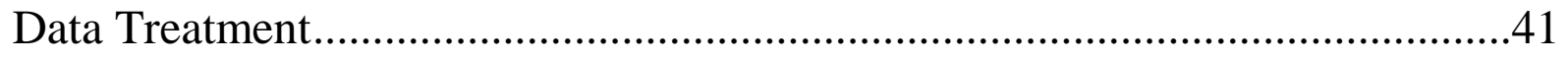

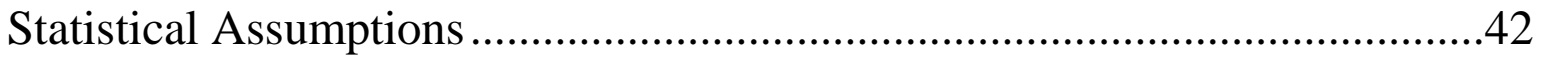


Results

Aim 1:.

Aim 2:.

Aim 3:.

Aim 4:

Aim 5:

Aim 6:

Aim 7:

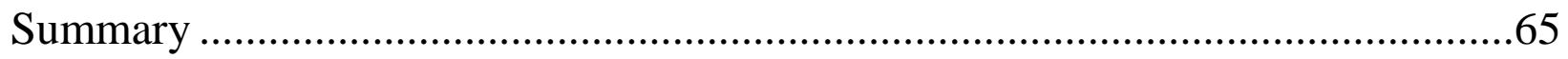

CHAPTER 5: SUMMARY, DISCUSSION, AND IMPLICATIONS ...................66

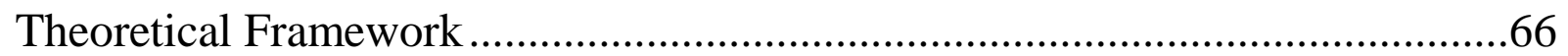

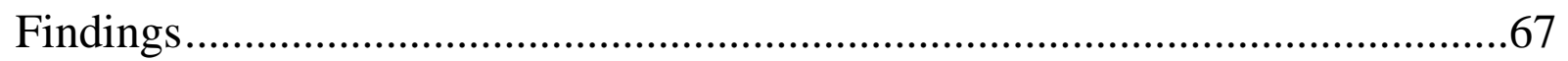

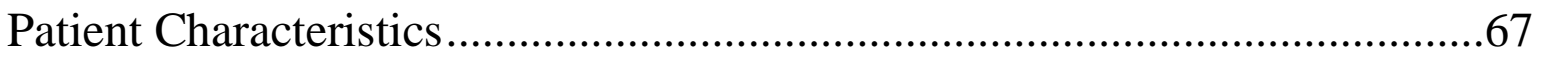

Biophysical Outcomes Prior to Intervention ................................................70

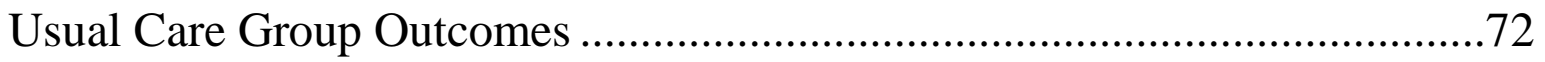

DGMV Intervention Group Outcomes ......................................................

Comparison of Usual Care and DGMV Intervention group outcomes ...........74 
Implications for Practice

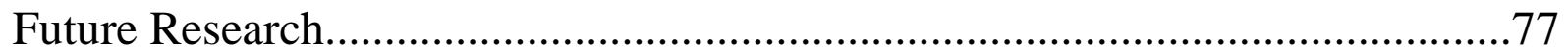

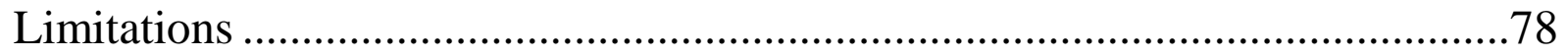

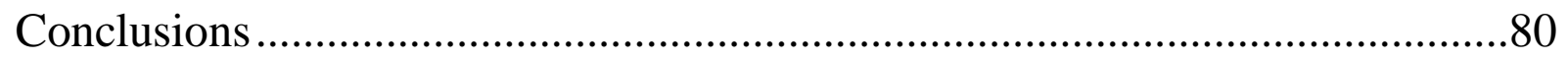

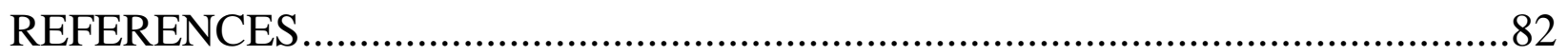




\section{LIST OF FIGURES}

Figure 1: Quality Health Outcomes Model........................................................................... 7

Figure 2: Conceptualization of QHOM for DGMVs ........................................................... 22

Figure 3: Deconstructed Model for Proposed Study ......................................................... 23

\section{LIST OF TABLES}

Table 1: Sample Characteristics Categorical Variables....................................................... 46

Table 2: Sample Characteristics Continuous Variables........................................................ 47

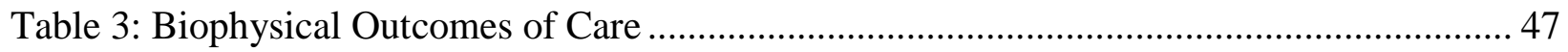

Table 4: Biophysical Outcomes of Care Before and After DGMVs ..................................... 50

Table 5: Biophysical Outcomes of Care for Usual Care Patients at Baseline and After One Year

Table 6: Differences in Biophysical Outcomes in Those Who Attend DGMVs versus Usual Care

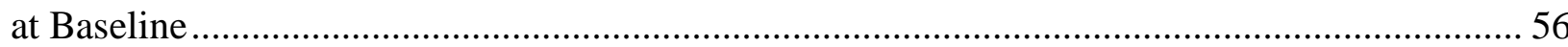

Table 7: Differences in Biophysical Outcomes in Those Who Attend DGMVs versus Usual Care

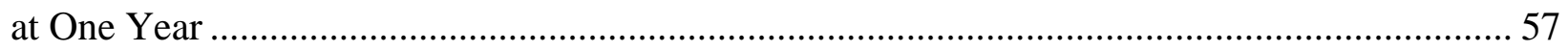

Table 8: Changes in Biophysical Outcomes in Those Who Attend DGMVs versus Usual Care at

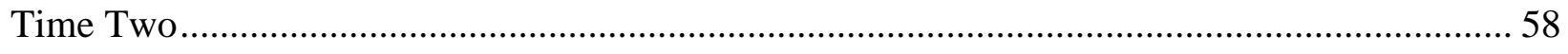

Table 9: Differences in Characteristics of DGMVs versus Usual Care Using $\mathrm{X}^{2} \ldots \ldots \ldots \ldots \ldots \ldots \ldots . . . \ldots 1$

Table 10: Differences in Characteristics of DGMVs verses Usual Care Using Independent t-tests

Table 11: Correlation between Dose of DGMVs and Biophysical Outcomes .......................... 64

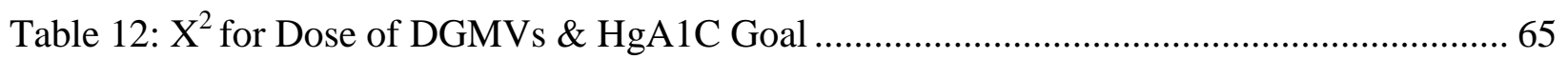




\section{LIST OF APPENDICIES}

Appendix A: Q-Q plots for differences in means of biophysical outcomes Aim 3 ................... 92

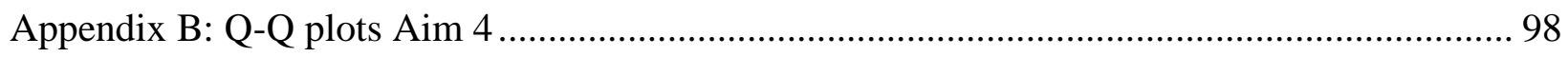

Appendix C: Q-Q plots for Aim 5 Usual Care .............................................................. 104

Appendix D: Q-Q plots for Aim 5 DGMVs .............................................................. 116

Appendix E: Q-Q Plots for Continuous Characteristics for Attendees of DGMVs .................. 128

Appendix F: Q-Q Plots for Continuous Characteristics for Usual Careare .............................. 130 


\section{CHAPTER 1: INTRODUCTION}

Diabetes mellitus is one of the major causes of morbidity and mortality in the United States with 1.6 million new cases diagnosed in people 20 years or older every year (Centers for Disease Control, 2007). If poorly managed, diabetes increases an individual's risk of lifelong health complications including blindness, chronic kidney disease, lower-limb amputations, peripheral neuropathy, decreased quality of life, decreased functional status, and emotional distress (Prevention, 2007). In 2007, the Centers for Disease Control estimated that diabetes cost the United States \$174 billion in both direct and indirect medical costs (Centers for Disease Control, 2007). It is estimated that nearly 23.6 million people have diabetes in the United States (Centers for Disease Control, 2007).

Data collected from the 2008 Behavioral Risk Factor Surveillance System (BRFSS) indicate that 14.7 percent of Americans are uninsured (Prevention, 2007). According to the 2006 National Health Information Survey (NHIS), 16.5\% of persons with diabetes reported that they needed medical care and did not receive it due to cost. Uninsured adults with diabetes predominantly have low incomes, are members of minority groups, and receive fewer preventive services than those with health insurance (Ayanian, Weissman, Schneider, Ginsburg, \& Zaslavsky, 2000). Hence, diabetes is a significant problem for the uninsured because untreated diabetes can lead to devastating consequences.

Diabetes mellitus accounts for a significant proportion of the care provided by primary health care providers (Mazze RS, 1994). Due to the complexities of managing diabetes, the American Diabetes Association (ADA) offers a foundation by which providers direct their medical treatment. Despite increasing provider knowledge, advancing treatment options, and providing countless educational programs, adherence to treatment regimens continue to be less 
than optimal (SERVICES, Prevention, \& Statistics, 2008). The treatment of diabetes is dependent not only upon knowledge and awareness of the provider, but on the knowledge, awareness, and situation of the patient (Barud, Marcy, Armor, Chonlahan, \& Beach, 2006). Current theoretically based research supports that provider approach is a key component affecting diabetes management. However, research supports that patient education, socioeconomic factors, and amount of social support may also affect diabetes management (Maddigan, Majumdar, \& Johnson, 2005). Substantial expenditure of healthcare dollars is incurred by people with diabetes. Resources could be saved by a reduction in diabetes comorbidities and complications. Reduction in resource use could be accomplished through improved diabetes care and outcomes. Cost-effective programs need to be initiated to maximize health gains for patients and to reverse the advance of this epidemic in society (Ryan, 2009). Specifically, peer support has been suggested to have the potential to provide a culturally appropriate exchange of resources between patients, aimed at increasing the well-being of the recipient of care (van Dam et al., 2005). Group medical visits, a type of peer support, have been used in recent years to improve the process of providing care and to improve outcomes for patients. However, there is a considerable gap in current knowledge about the effectiveness of Diabetes Group Medical Visits (DGMVs) especially related to the dose of visits needed to impact outcomes.

\section{Significance}

DGMVs have the potential to positively affect outcomes of care for those who are uninsured (Clancy, Brown, Magruder, \& Huang, 2003; Trento et al., 2002; Wagner et al., 2001). Little is known about the characteristics of uninsured persons with diabetes, who attend or will benefit from group visits, and the dosage of group visits that is needed to affect outcomes of care. Group visits may 
offer more intensive care to uninsured individuals by clustering care and providing peer support during the visit.

Patients have reported that group visits are an acceptable form of health care, and minority populations have found that group visits are culturally relevant (Keyserling et al., 2000). Research has documented that persons with diabetes who participate in group visits had increased satisfaction with care, interaction with providers, diabetes knowledge, education, quality of life, preventive procedures and screenings (Wagner et al., 2001; Trento et al., 2001). Clinical outcomes associated with group visits in persons with diabetes have been documented to include: decreased or stable $\mathrm{HgA1C}$, decreased cardiovascular risk, decreased or stable BMI, decreased LDL, increased HDL, decreased or stable blood pressure and slowed progression of retinopathy (Wagner, et al., 2001). All of these positive clinical outcomes were achieved with an increase in provider trust and a decrease in or more effective use of provider time (Clancy et al., 2003). However, the dosage of DGMVs that may affect outcomes of care is unknown.

This proposed study will attempt to increase knowledge related to the effect of DGMVs on those who are uninsured, the dosage of DGMVs that may affect outcomes of care, and how biophysical outcomes of care are impacted by DGMVs. This knowledge can potentially impact practice, resource utilization, and research for the future.

\footnotetext{
Aims

The purpose of this descriptive, correlational study was to analyze the relationship between attendance at Diabetes Group Medical Visits (DGMV) and biophysical outcomes of care in uninsured persons with diabetes who are cared for in a free clinic. Specifically the aims of this study will be:
} 
1. To describe the characteristics of uninsured persons with diabetes who are cared for in a free clinic.

2. To describe the biophysical outcomes of care in uninsured persons with diabetes who are cared for in a free clinic.

3. To compare biophysical outcomes of care in persons with diabetes before attending DGMVs and one year after attending DGMVs.

4. To compare biophysical outcomes of care in uninsured persons with diabetes who receive usual care in a free clinic at baseline and after one year.

5. To analyze the differences in biophysical outcomes in persons with diabetes who attend DGMVs versus those who receive usual care in a free clinic at base line and then again after one year.

6. To analyze the differences in characteristics of uninsured persons with diabetes who are attendees of DGMVs versus those who receive usual care.

7. To explore the impact of dose of DGMVs on biophysical outcomes of care in uninsured persons with diabetes who receive care in a free clinic after one year. 


\section{CHAPTER 2: REVIEW OF LITERATURE}

The purpose of this chapter is to explore the literature related to Diabetes Group Medical Visits (DGMVs) and biophysical outcomes of care. First, the Quality Health Outcomes Model (QHOM), the theoretical framework guiding this study, will be presented. Then, the QHOM will be used as a guide to present the current empirical literature related to DGMVs and biophysical outcomes of care for persons with diabetes.

\section{Conceptual Framework: Quality Health Outcomes Model}

The theoretical framework guiding this study is the Quality Health Outcomes Model (QHOM). The QHOM was developed by the American Academy of Nursing's Expert Panel on Quality Health Care in 1996 as an expansion of Donabedian's structure-process-outcome framework. The QHOM is a more dynamic framework that acknowledges the feedback that occurs between patients, the system or context in which care is provided, and interventions (Mitchell, Ferketich, \& Jennings, 1998). This model links outcomes to the interactions of patients and the healthcare system with healthcare interventions intended to treat the individual, family, or community (Mitchell, Heinrich, Moritz, \& Hinshaw, 1997). The four major concepts included in this model are: system, interventions, patients, and outcomes. These concepts have reciprocal connections, except for interventions and outcomes. Factors that may affect patient outcomes include the three major concepts of the QHOM: system, interventions, and patient characteristics (see Figure 1). Interventions affect and are affected by both system and patient characteristics in producing desired outcomes.

The QHOM guides the identification of factors contributing to patient outcomes, which is the focus of this study. The model posits that outcome measures are the result of care structures that integrate functional, social, psychological, physical and physiologic aspects of people's 
experience in health and illness. The model further proposes that such outcome measures should be operationalized in five categories: achievement of appropriate self-care, demonstration of health-promoting behaviors, health-related quality of life, perception of being well-cared-for, and symptom management (Mitchell, et al., 1998).

The purpose of this study was to analyze the relationship between attendance at Diabetes Group Medical Visits (DGMVs) and biophysical outcomes of care in uninsured persons with diabetes who are cared for in a free clinic. The QHOM is used as the theoretical underpinning for this study. Therefore, the literature will be reviewed and presented by the major concepts of the QHOM: intervention, patient characteristics, and outcomes of care. 
Figure 1: Quality Health Outcomes Model

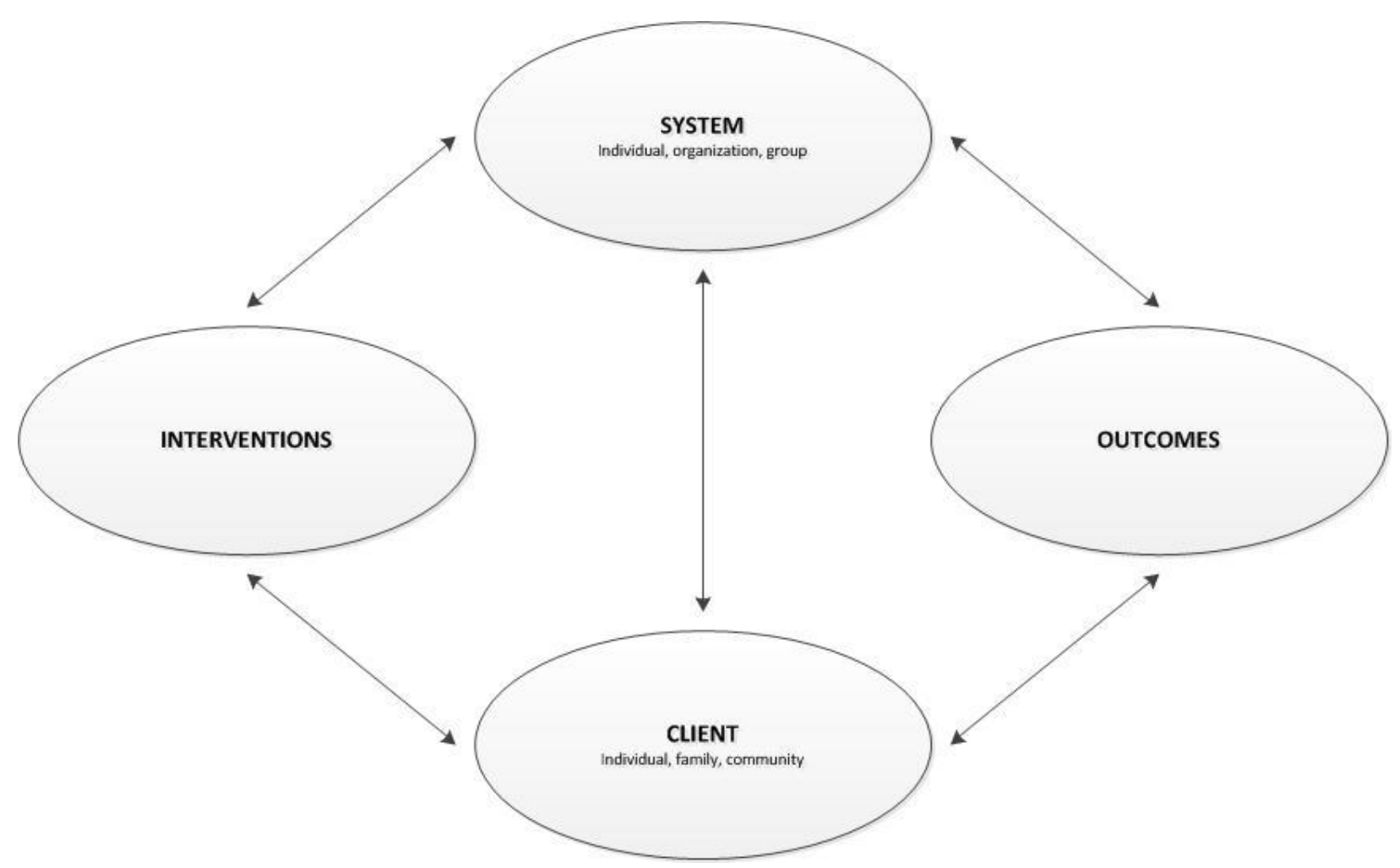

(Mitchell, et al., 1998) 


\section{Literature Review}

In this section the empirical literature related to DGMVs will be reviewed. In order to complete the review, three computerized databases (Pubmed, Medline, and CINAHL) were searched using the key words of diabetes, peer support, social support, support groups, group visits, quality health outcome model, chronic care model, cooperative health care clinics, and drop-in group medical appointments. Limits were set for articles published in the English language. A total of 624 articles were identified that met these requirements. Group medical visits have only emerged as a treatment option for persons with diabetes over the past decade. Hence, limits were set for articles published in the last ten years. Limits for empirical literature in outpatient settings were also added. In addition, the bibliographies of these studies, as well as review articles on adherence, were examined for additional references. Review articles, editorials, and practice models were not included. Using these limits a total of 37 articles were reviewed. The quality of the articles was evaluated by using the Rosswurm \& Larrabee critique guidelines (Rosswurm \& Larrabee, 1999). The data abstraction was completed systematically by the author using a matrix system.

\section{System Characteristics}

System characteristics are traditional structure and process elements of organizations, such as size, ownership, skill mix, and technology. There are three levels of system characteristics including individual, group, and organizational levels. The system characteristics in the QHOM are considered to directly affect and be affected by patient outcomes. The system characteristics are the mediators of patient characteristics and interventions in producing patient outcomes (Mitchell, et al., 1998). 
The system of interest in this study is the free clinic. The National Association of Free Clinics defines a free clinic as a volunteer-based, safety-net health care organization that provides a range of medical and/or behavioral health services to economically disadvantaged uninsured individuals. Free clinics serve mostly nonelderly adults, women, and minorities with low incomes(Darnell, 2010). There are over 1000 free clinics in the United States that provided care for 1.8 million individuals (Darnell, 2010). Free clinics focus on providing services less readily available to those without insurance such as medications and health education (Darnell).

\section{Intervention}

The intervention of interest in this study is the diabetes group medical visit. Mitchel et al, (1998) posit that clinical processes, such as delivering care thorough a DGMV, are direct and indirect interventions, however there is no single, direct connection linking interventions and outcomes. The model suggests a reciprocal direction of influence. Interventions affect and are affected by both system and patient characteristics in producing desired outcomes. The effect of an intervention is mediated by the patient and the system characteristics.

\section{Group Medical Visits}

A group medical visit is defined as any visit that attempts to provide group education while providing health care at the same time (Bray, 2005). This differs from group diabetes education where patients may receive group education but do not receive health care. Studies reviewed focused on DGMVs as an intervention and evaluated non-biophysical outcomes such as feasibility, cultural relevance, acceptability, efficacy, perceptions of care, self-care behaviors, trust in provider, cost, patient satisfaction, receipt of preventative services, quality of life, knowledge, locus of control, and self-management support. 
A descriptive study was published by Keyserling et al. (2000) with the purpose of examining the feasibility of an intervention program which included group medical visits designed to improve dietary physical activity, and self-care behaviors of older African American women with diabetes. The sample consisted of 200 African American women with diabetes. Focus groups were conducted to determine the cultural relevance and acceptability of the intervention. The authors report that the participants found group visits to be culturally relevant and acceptable. The effect of group visits on diet, physical activity, and self-care behaviors were not studied. The feasibility of group visits as an intervention is supported by a study conducted by Clancy, Cope, Magruder, Huang, Salter et al. (2003). The purpose of their experimental study was to evaluate the feasibility and acceptability of group visits to uninsured or inadequately insured patients with uncontrolled diabetes. Participants were predominantly African American (77.5\%) and most of the participants had health-care insurance (73.1\%). Clancy and colleagues (2003) found group visits to be feasible and acceptable to patients with uncontrolled diabetes and fostered an improved sense of trust in their physician. More recently, a descriptive study was published by Barud, Marcy, Armor, Chonlahan, \& Beach (2006). The authors evaluated the effectiveness of group medical visits for persons with diabetes in one family medicine center. The effectiveness of the group visits was evaluated by a 12-question patient satisfaction survey completed at the close of each group session. The survey was used to evaluate patient satisfaction with the experience at the center. Participants reported that group visits helped clinicians provide them with more efficient health-care and education than traditional care. Not only have group visits been found to provide more efficient care, they have also been shown to increase positive perceptions of health-care. Clancy, Yeager, Huang, \& Magruder (2007) conducted a study to evaluate perceptions of care delivered through group visits to disadvantaged 
patients with diabetes. The design of the study was experimental. A total of 120 persons with diabetes were randomly assigned to receive their care in group visits or usual care for six months. After six months, patient satisfaction was measured by survey. The sample was $72 \%$ female and $82 \%$ African American. The authors reported that patients assigned to group visits had generally more positive perceptions about their care in the areas of ongoing care, community orientation of care, and cultural competence of care than did those in usual care. However, one study did find that telephone management of diabetes may be better for some patients.

Schillinger et al. (2007) attempted to describe the difference in self-management support systems in the dimensions of participation, representativeness and engagement between group visit participants and patients receiving individual support through telephone disease management. The study found that telephone disease management yielded higher engagement, especially among those with limited English proficiency and limited literacy. However, no statistical testing for differences in engagement between telephone disease management and group medical visits was performed. In summary, this literature review supports that DGMVs have the potential to impact outcomes of care.

\section{Outcomes of Care}

An outcome is said to be a component of a patient's clinical and functional status after an intervention has been applied (Barr, Schumacher, \& Myers, 2001). The QHOM proposes that outcome measures should be the result of care structures and processes that integrate functional, social, psychological, physical, and physiologic aspects of people's experience in health and illness (Mitchell et al., 1998). Studies reviewed evaluated common outcomes measured in diabetes populations outcomes of care such as $\mathrm{HgA1C}$, weight, blood pressure, fasting glucose, lipids, hypoglycemic episodes, anxiety, depression, end organ damage, and microalbumin 
(Clancy, Brown, et al., 2003; Clancy, Yeager, Huang, \& Magruder, 2007; Culhane-Pera et al., 2005; Trento, et al., 2002; Trento et al., 2001; Wagner, et al., 2001). Additionally, some studies evaluated both biophysical outcomes as well as non-biophysical outcomes of care.

\section{Biophysical Outcomes of Care}

While $\mathrm{HgA} 1 \mathrm{C}$ is commonly used to determine an improvement in glucose levels in persons with diabetes, multiple other outcomes measures can be used as indicators of improved care. Trento et al. (2002) published the results of a randomized controlled clinical trial to compare traditional individual diabetes care with a model in which routine follow-up was managed by interactive group visits. The study found that $\mathrm{HgA} 1 \mathrm{C}$ increased in the usual care group but not in the group visit patients; those participating in group visits had a decrease in their BMI and an increase in HDL. In addition, the dosage of hypoglycemic agents decreased and retinopathy progressed less among the group care patients. Diastolic blood pressure and relative cardiovascular risk decreased from baseline in both the group visit and usual care patients. More biophysical outcomes of care were looked at by Kirsh et al. (2007). The purpose of the study by Kirsh and colleagues (2007) was to evaluate the impact of shared medical appointments on intermediate outcome measure of care for persons with diabetes focusing on those patients at highest cardiovascular risk. Chart reviews were conducted to collect data. The findings suggested that reductions in $\mathrm{HgA1C}$, LDL, and systolic blood pressure were greater in the intervention group but the difference was not statistically significant. Another study which looked at only biophysical outcomes had similar findings. A quasi-experimental study published by Bray, Thompson, Wynn, Cummings, \& Whetstone (2005) explored the efficacy of combining care management and interdisciplinary group visits for rural African American persons with diabetes mellitus. The vast majority of participants were African American (90\%). Most patients had 
health insurance through Medicare or Medicaid (83\%). The intervention group had a significant decrease in $\mathrm{HgA} 1 \mathrm{C}$ versus the control group compared over the same time period. No significant differences in mean weight or blood pressure between group visits and usual care were found.

\section{Biophysical Outcomes \& Diabetes Group Medical Visits as an Intervention}

Biophysical outcomes do not always improve when using DGMVs as an intervention. Clancy, Cope, Magruder, Huang, \& Wolfman (2003) published results of an experimental study to evaluate the effectiveness of a managed care approach to health care delivery, i.e. group visits, in the management of uninsured or inadequately insured patients with diabetes. The participants were predominantly female (78.3\%) and African American (77.5\%). Most of the participants had health-care insurance (73.1\%). The data collected were those charted as ADA standards of care: HgA1C and lipids over 6 months. Group visits were found to be more effective in promoting documentation of concordance with ADA standards of care than usual care. However despite the innovative delivery method, there were no significant differences seen in diabetes or lipid control. Five years later, Clancy, Huang, Okonofua, Yeager, \& Magruder (2007) published a study with similar findings. The study evaluated the effect of group visits on documentation of clinical outcomes in concordance with 10 American Diabetes Association guidelines. The study was an experimental design with measures of ADA standards of care at 6 and 12 month intervals. The sample consisted of 186 patients with diabetes, predominantly female (72\%) and African American (82\%). Findings of the study at both measurement points were that HgA1C, blood pressure, and lipid levels did not differ significantly for patients attending group visits versus those receiving usual care. At 12 months, patients in group visits exhibited greater concordance with ADA process of care indicators and rates for cancer screening patients. The authors suggest that modification to the content and style of group visits may be necessary to 
achieve improved clinical outcomes. Another study that measured biophysical and nonbiophysical outcomes of care had comparable results. Culhane-Pera et al. (2005) published the results of a study with the purpose of evaluating the influence of group visits on diabetes management in Hmong adults with diabetes. The hypothesis of this study was that group visits would improve diabetes management. The authors reasoned that having a forum to discuss diabetes in a culturally familiar group setting would facilitate people's acceptance of clinical services, medications, and lifestyle changes. The study findings were that participants received better services; however, biological parameters and mental health did not improve.

One study has shown positive effects of group visits for both biophysical and nonbiophysical outcomes of care. Wagner et al. (2001) published the results of a quasi-experimental study with 707 diabetes patients. The purpose of the study was to evaluate the impact of primary care group visits on the process and outcome of care for diabetic patients. The findings of the study showed the intervention group had received significantly more recommended preventive procedures and patient education. There was a consistently positive association between the number of group visits attended and outcomes, such as patient satisfaction and HgA1C levels.

Cost can also be decreased through implementation of group visits. Bray, Roupe et al. (2005) conducted a study to assess the feasibility and potential for cost effectiveness of restructuring care in rural fee-for-service practices for predominantly minority persons with diabetes. The majority of patients were African American (72\%) and more than half of the patients were female (54\%). The major findings of the study were an improvement in the percentage of patients achieving diabetes management goals. Additionally, there was improvement in the providers' productivity and billable encounters. Increased monetary revenue can be accompanied by decreasing workloads of providers. Trento et al. (2001) conducted a 
study to evaluate whether group visits in diabetes care are more effective than individual consultations in improving self-care and metabolic control. The hypothesis of their quasiexperimental study was that individual visits could be merged with interactive group visits into a permanent therapeutic educational process, including interactive techniques, positive group dynamics and identification with other group members, without increasing the workloads of health care providers in outpatient diabetes care. The authors reported that participants in group consultations had stable $\mathrm{HgA} 1 \mathrm{C}$ levels as compared to increased $\mathrm{HgA} 1 \mathrm{C}$ levels in control group. The intervention group had lower cardiovascular risk scores, improved diabetes knowledge, better quality of life, improved health behavior scores and longer interaction with health-care providers, while the physicians spent less time seeing the group rather than completing individual appointments.

Diabetes group medical visits have been studied in various patient populations and numerous disciplines. Both qualitative and quantitative designs have been used to study this model for care. Qualitatively, researchers have studied diabetes group medical visits though interviews usually focusing on quality of life. Typically, in quantitative studies, health outcomes are measured to determine effectiveness of group visits. The outcomes measured vary from study to study. Frequently, studies that use group medical visits as an intervention, measure outcomes of adherence such as body mass index, weight, glycosylated hemoglobin, fasting blood glucose, blood creatinine, lipids, microalbuminuria, blood pressure, evidence of end organ damage, depression, anxiety, medication use, and foot care. Other studies evaluated process of care indicators such as prescribed ACE inhibitors, aspirin, cholesterol management, cholesterol treatment, measurement of glycosylated hemoglobin, measurement of microalbumin, Pneumovax administration, influenza vaccine administration, eye examination, and foot 
examination (Chiu et al., 2009; Clancy, Brown, et al., 2003; Clancy, Yeager, et al., 2007; Culhane-Pera, et al., 2005; Guzek, Guzek, Murphy, Gallacher, \& Lesneski, 2009; Trento, et al., 2002; Trento, et al., 2001; Wagner, et al., 2001). Yet other studies have measured system characteristics such as the cost effectiveness, productivity and billable encounters of diabetes group medical visits (Bray et al., 2005; Clancy, Brown, et al., 2003; Clancy, Dismuke, Magruder, Simpson, \& Bradford, 2008). Other than HgA1C, no two researchers found in this review, measured the same outcomes of care in persons with diabetes who received group medical visits or used the same measurement tools. The literature supported outcome measures that were used for the proposed study include body weight, body mass index, fasting blood glucose, $\mathrm{HgA1c}$, serum creatinine, lipids, blood pressure and microalbumin.

\section{Patient Characteristics}

Mitchell and colleagues (1998) state that patient characteristics are factors that directly affect outcomes and include client health, demographics, and disease risk factors. Age, gender, ethnicity, marital status, duration of diabetes, education, distance from clinic, co-morbidities, and depression are all patient characteristics commonly measured in diabetes group medical visits (Barud, et al., 2006; Bray, Roupe, et al., 2005; Bray, Thompson, Wynn, Cummings, \& Whetstone, 2005; Clancy, Brown, et al., 2003; Clancy, et al., 2008; Clancy, Yeager, et al., 2007; Culhane-Pera, et al., 2005; Keyserling, et al., 2000; Schillinger et al., 2007; Trento, et al., 2002). Examining patient characteristics may identify which patient populations for which DGMVs are most beneficial.

Age

Age of patients has been associated with self-management activities, thus affecting outcomes of care. The World Health Organization (2003) reports that in most studies of 
adherence, age of the patient was associated with outcomes of care (Sabate, World Health, Project, \& Global Adherence Interdisciplinary, 2003). Leventhal et. al, (2001) reported that compared to younger participants, persons with diabetes over the age of 25 reported exercising on fewer days per week, and spending less time in recreational physical activities. Older adults may also practice better self-management than younger adults (Leventhal H, 2001). Additionally, the fact that glucose intolerance increases with age has been apparent for over 30 years (Andres, 1971).

\section{Ethnicity}

Other patient characteristics such as ethnicity can affect outcomes as well. The rate of diabetes is increasing fastest in ethnic minorities, including African Americans, Mexican Americans, and Native Americans (Promotion, Accessed September 20, 2010). Because of cultural differences, ethnicity is reported to be a risk factor for poorer quality in health care, disease management and disease control. Ethnic minority groups are at risk for poorer quality of life and increased disease complications when compared with non-ethnic counterparts living in the same country (Mc Manus \& Savage). Mitchell and colleagues (1998) suggested that client characteristics, such as ethnicity have a meaningful, direct effect on behavioral and health status outcomes. It is believed that clients live in a social environment with cultural values and beliefs about health and healthcare (Mitchell, et al., 1998). These values and beliefs affect the patients' desire to interact and ability to interact with a care delivery system (Holzemer, 1994).

\section{Comorbidities}

The majority of older adults have two or more chronic conditions and among patients with diabetes, $40 \%$ have at least three (Sabate, et al., 2003). Patients with a greater overall number of comorbidities place lower priority on diabetes and have worse diabetes self- 
management ability scores (Glasgow, Toobert, \& Hampson, 1996). The type and severity of comorbid conditions can affect outcomes of care. Patients with severely symptomatic comorbidities and those with conditions they consider to be unrelated to diabetes may need additional support in making decisions about care priorities and self-management activities (Kerr et al., 2007).

One co-morbidity that may affect outcomes of care in persons with diabetes is depression. The incidence of depression has been observed to be twice as high among persons with diabetes than in the general population (J. J. Prochaska, Nigg, Spring, Velicer, \& Prochaska). Patients with depression are more likely to experience complications of diabetes, have worse glycemic control, and be less adherent to self-care behaviors than patients who are not depressed (J. O. Prochaska, 2008).

\section{Other Patient Characteristics}

Other patient characteristics such as gender, duration of diabetes, miles to clinic, and educational level also are suggested to affect outcomes of care. Gender has been associated with outcomes such that men with diabetes have been found to be more physically active than women with diabetes, but they also consume more calories, eat more inappropriate foods and have lower levels of adherence (Whitlock, Vogt, Hollis, \& Lichtenstein, 1997). Duration of disease appears to have a negative relationship with adherence: the longer a patient has had diabetes, the less likely he or she is to be adherent to treatment (Glasgow, Davidson, Dobkin, Ockene, \& Spring, 2006). Longer driving distances from home to the site of primary care were associated with poorer glycemic control in older, rural subjects (Strauss, MacLean, Troy, \& Littenberg, 2006). Educational level has been shown to be significant in disease control of diabetes patients. On the National Health and Nutrition Examination Survey from 1999 to 2006 those persons with 
diabetes who are more educated have consistently shown an improved $\mathrm{HgA1C}$, blood pressure and total cholesterol level than those who are less educated (McWilliams, Meara, Zaslavsky, \& Ayanian, 2009). Education level and health literacy also appear to have an effect on participation in medical decision making and thus may impact outcomes of care (DeWalt, Boone, \& Pignone, 2007). Patient characteristics that will be examined in the proposed study are therefore, age, gender, ethnicity, marital status, duration of diabetes, education miles form clinic, comorbidity, and depression.

\section{Synthesis}

Group visits for persons with diabetes have been found to be feasible (Keyserling, et al., 2000). This practice change has been implemented in a variety of clinical systems. Patients have reported that group visits are an acceptable form of health care, and minority populations find that group visits are culturally relevant (Keyserling, et al., 2000).

Feasibility, cultural relevance, acceptability, trust in provider, cost, self-management support, perceptions of care, satisfaction, receipt of preventive services, insurance status, and efficacy have been studied while providing DGMVs as an intervention. The outcomes of care that have been studied with DGMVs are HgA1C, weight, blood pressure, fasting glucose, lipids, hypoglycemic episodes, anxiety, depression, end organ damage, microalbumin, self-care behaviors, patient quality of life, knowledge, ethnicity, and locus of control. Patient characteristics commonly studied in relation to DGMVs have been age, gender, ethnicity, marital status, duration of DM, education, miles from clinic, co-morbidities and depression.

Research documented that persons with diabetes who participate in group visits as an intervention experience an increase in the following: satisfaction with care, interaction with providers, diabetes knowledge, education, quality of life and preventive procedures and 
screenings (Trento, et al., 2001; Wagner, et al., 2001). Clinical outcomes associated with group visits have been documented to include: decreased or stable $\mathrm{HgA1C}$, decreased cardiovascular risk, decreased or stable BMI, decreased in LDL, increased in HDL, decreased or stable blood pressure and slowed progression of retinopathy (Clancy, Cope, et al., 2003). Positive clinical outcomes have been achieved with an increase in provider trust and a decrease or more effective use of provider time (Clancy, Cope, et al., 2003). However, worsening of diabetes control is only found to have been prevented in studies where patients had peer support and consultation with a physical or other health care provider (Philis-Tsimikas et al., 2004; Trento, et al., 2001).

While these findings regarding group visits are promising, some major limitations in the evidence still exist. Random assignment to treatment was rare in the studies reviewed. Because most subjects volunteered to be involved in the treatment, selection bias may have occurred, as highly motivated patients are more likely to volunteer. The majority of the participants in each study were from ethnic minority groups and female. All but one study focused on participants in their $5^{\text {th }}$ and $6^{\text {th }}$ decades of life. Most of the participants had some type of health care insurance. While these studies add to the body of knowledge regarding group medical visits for persons with diabetes, lack of randomization and lack of heterogeneity limits generalizability of the findings.

The purpose of this study was to analyze the relationship between attendance at Diabetes Group Medical Visits (DGMVs) and biophysical outcomes of care in uninsured persons with diabetes who are cared for in a free clinic. The conceptualization of the QHOM for the purposes of this study is shown in Figure 2. The system characteristic in this study was a free clinic for those who are uninsured in North Central West Virginia. This system characteristic remained constant for all subjects. The intervention will be Diabetes Group Medical Visits. The patient 
characteristics studied were age, gender, ethnicity, marital status, duration of DM, education, miles from clinic, co-morbidities, and depression. The outcomes measured were body weight, BMI, fasting blood glucose, $\mathrm{HgA1C}$, creatinine, lipids, blood pressure, and microalbumin. All outcomes were compared before and after group medical visits, as well as to a group of patients in the same system who received traditional care. 
Figure 2: Conceptualization of QHOM for DGMVs

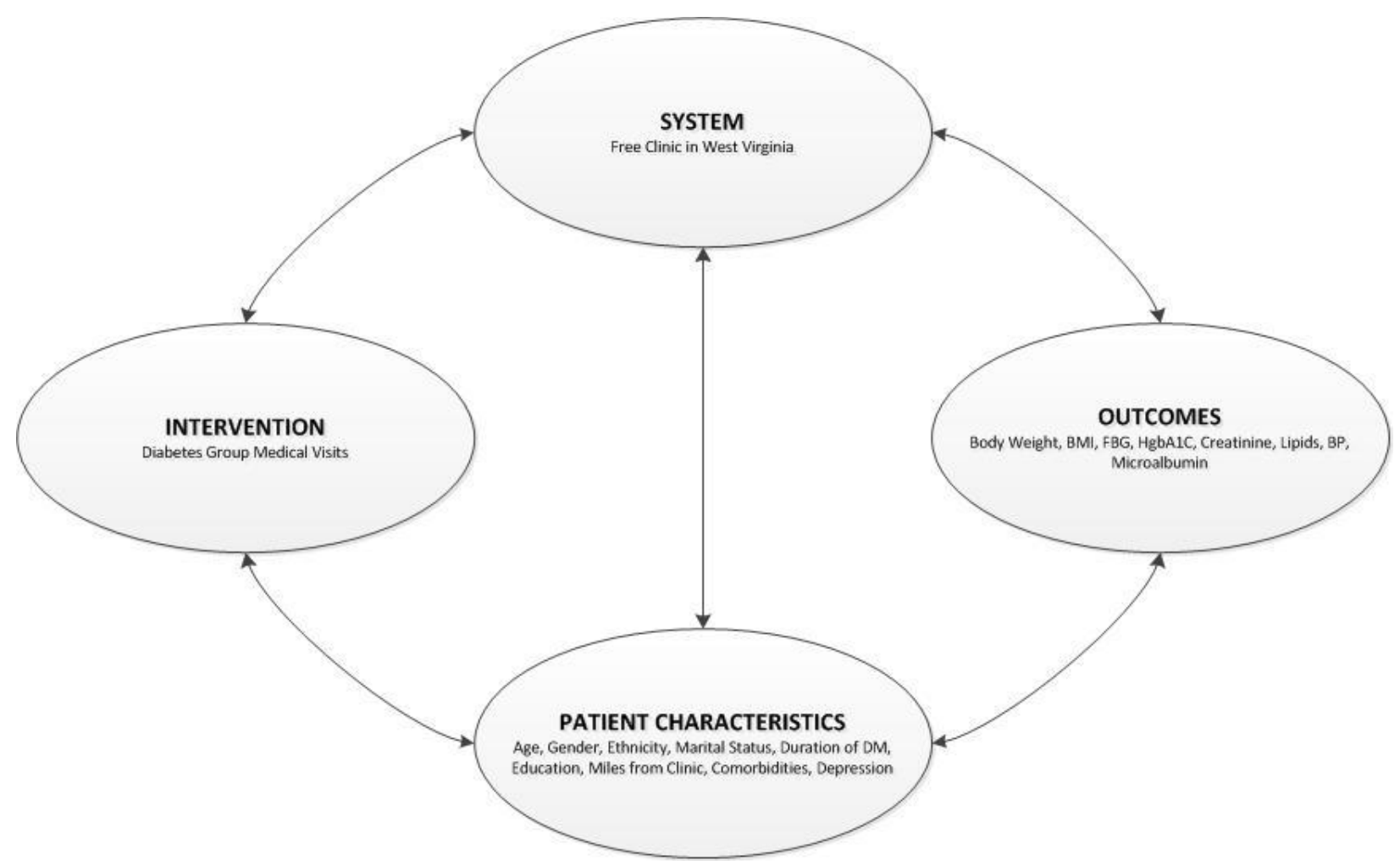


Figure 3: Deconstructed Model for Proposed Study

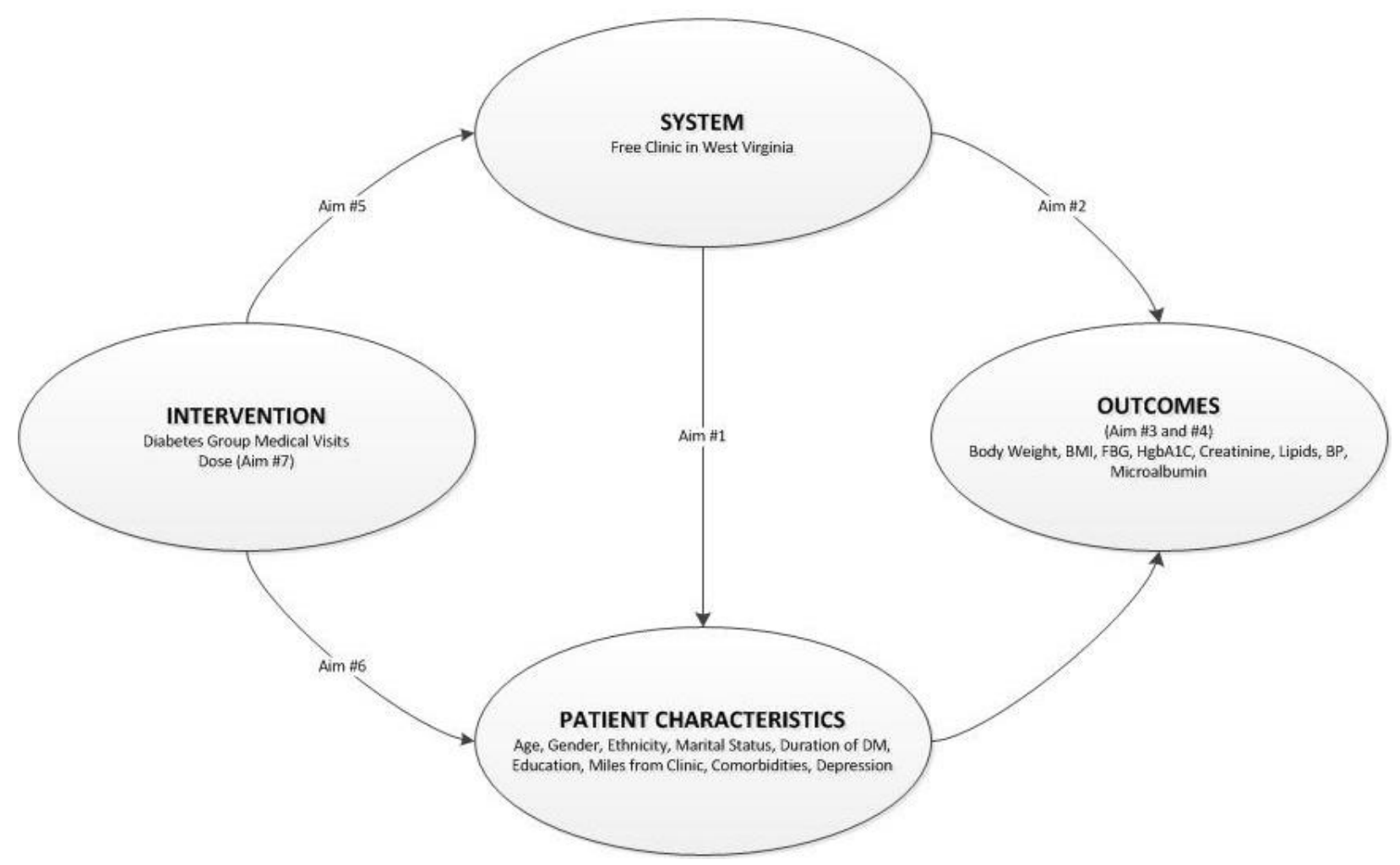




\section{CHAPTER 3: METHODOLOGY}

The purpose of this study was to examine the relationship between attendance at Diabetes Group Medical Visits (DGMV) and biophysical outcomes of care in uninsured persons with diabetes who receive care in a free clinic. Specifically the aims of this study were:

1. To describe the characteristics of uninsured persons with diabetes who are cared for in a free clinic.

2. To describe the biophysical outcomes of care in uninsured persons with diabetes who are cared for in a free clinic.

3. To compare biophysical outcomes of care in persons with diabetes before attending DGMVs and one year after attending DGMVs.

4. To compare biophysical outcomes of care in uninsured persons with diabetes who receive usual care in a free clinic at baseline and after one year.

5. To analyze the differences in biophysical outcomes in persons with diabetes who attend DGMVs versus those who receive usual care in a free clinic at base line and then again after one year.

6. To analyze the differences in characteristics of uninsured persons with diabetes who are attendees of DGMVs verses those who receive usual care.

7. To explore the impact of dose of DGMVs on biophysical outcomes of care in uninsured persons with diabetes who receive care in a free clinic after one year.

This chapter conceptually and operationally defines measures used to meet the study aims and describes study methods. 


\section{Variable Definitions}

\section{Diabetes Group Medical Visits}

\section{Conceptual Definition}

The group medical visit model was developed in managed care to improve effectiveness of care of patients (Clancy, Huang, Okonofua, Yeager, \& Magruder, 2007). Mental health providers and behavioral therapists have long recognized the value of groups when seeking improved psychological and behavioral outcomes for people with chronic illnesses (Beck et al., 1997). Group interaction appears to provide emotional support while lessening feelings of isolation and stigmatism that are associated with some chronic illnesses (Weinger, 2003). A group medical visit must include at least two patients, who are united in a health-related situation, and a medical provider (Barud, et al., 2006). Each patient must share similar experiences and be willing to participate in a group. All parties need to possess the ability to receive and respond to social interaction. Group participants must also possess specific knowledge derived from personal experience. All group medical visits include some degree of information, appraisal and emotional support (Dennis, 2003). Additionally, some form of health care provider is present. In this, group visits differ from other forms of group interventions, such as support groups or diabetes education, which are generally led by peers and do not include oneon-one consultations with a health care provider.

\section{Operational Definition}

In the free clinic where this study took place, the DGMV was an additional health care visit and is meant to supplement individual healthcare visits to improve patient outcomes. Diabetes Group medical visits included group education and interaction and elements of an individual patient visit, such as the collection of vital signs, history taking, physical exam, 
medication adjustments, appropriate standardized referrals, and laboratory procedures related to diabetes care (Jaber, Braksmajer, \& Trilling, 2006). The curriculum for this clinic's DGMV, which was adapted from the American Diabetes Association's standards of care (AmericanDiabetesAssociation, 2010) was developed by the Nurse Practitioner at the clinic in conjunction with a Pharmacist (PharmD) who is obtaining certification in diabetes education. The free clinic offered up to six DGMVs in which the patients were provided education about blood glucose monitoring, medication, nutrition, exercise, foot care, heart disease, complications including sick day care, and behavior changes. The patients were scheduled to attend the clinic up to once a month until they had received all of the education offered by the DGMVs. Because the classes were offered four times per month, the patients could schedule at their convenience. Hence, the participants in each group varied from class to class. Patients were referred to the DGMV by their primary care provider for additional care. Attendance at the DGMVs was voluntary. Data related to the number of DGMVs were collected from the chart and was coded $1,2,3,4,5$ or 6 depending on the number of visits patients experienced. Data were also collected on which DGMVs the patient attended. The data were recorded as categorical for each of the following DGMV categories: blood glucose monitoring, medication, nutrition, exercise, foot care, heart disease, complications including sick day care, and behavior changes.

\section{Usual Care}

\section{Conceptual Definition}

Usual care can be defined as the routine care received by those patients who did not participate in DGMVs. The American Diabetes Association (ADA) has built the foundation by which providers direct their medical treatment for diabetes management. Ten established guidelines backed by research findings have become the standard for diabetes medical 
management. Recommendations for all persons with diabetes are to have annual retinal and foot exams, annual influenza vaccinations, and pneumococcal immunizations, and monitoring of blood pressure, urine microalbumin levels, lipid profiles, quarterly measurement of $\mathrm{HgA1C}$, and cardiovascular risk factor assessment with treatment as indicated for all abnormal results.

\section{Operational Definition}

Usual care for persons with diabetes in the free clinic where the study took place included collection of vital signs, history taking, physical exam, medication adjustments, appropriate referrals, laboratory procedures, and education provided by the health care provider related to general care. Usual care did not include education provided by a Pharm D or diabetes educator with a group of other diabetes patients at the time of the usual care visit. Data related to the number of care visits during the study period were collected from the chart as a continuous variable.

Biophysical Outcomes of Care

\section{Conceptual Definition}

An outcome is said to be a component of a patient's clinical and functional status after an intervention has been applied (Barr, et al., 2001). Biophysics refers to the process of assigning an objective measurement to a bodily process. For the purposes of this study, a biophysical outcome of care was defined as the measurable result of care collected over a specific time frame. Common biophysical outcomes measured in diabetes populations are body weight, body mass index (BMI), glycosylated hemoglobin ( $\mathrm{HgA1C}$ ), fasting blood glucose (FBG), serum creatinine, serum lipids, urine microalbumin, and blood pressure (Clancy, Brown, et al., 2003; Clancy, Yeager, et al., 2007; Culhane-Pera, et al., 2005; Trento, et al., 2002; Trento, et al., 2001; Wagner, et al., 2001). 


\section{Operational Definition}

This study collected the common biophysical outcomes measured in diabetes, body weight, BMI, $\mathrm{HgA1C}$, fasting blood glucose, serum creatinine, serum lipids, urine microalbumin, and blood pressure.

Body Weight

Body weight was obtained routinely and recorded in the chart at the beginning of each clinic visit. Weight was measured and recorded in pounds (lbs). The clinic used an upright mechanical medical scale with capacity to weigh patients up to $350 \mathrm{lbs}$. This study collected the first body weight available prior to May 2007 and the most recent body weight recorded on or before August 18, 2009. The weight of patients that weigh more than $350 \mathrm{lbs}$ is reported in the chart as "350+." These data were recorded and analyzed as a continuous variable.

\section{Body Mass Index}

Body mass index was calculated with the following formula: weight (lb) / [height (in) $]^{2} \mathrm{x}$ 703. Height was collected via patient report and recorded in the chart upon initial visit. Weight was obtained routinely and recorded in the chart at the beginning of each clinic visit. This study collected the first body weight available prior to May 2007 and the most recent body weight recorded on or before August 18, 2009. BMI was calculated based on the initial patient reported height. These data were entered as a continuous variable.

\section{Glycosylated Hemoglobin}

Glycosylated Hemoglobin levels were drawn as part of routine diabetes care. In this clinic the measurement of $\mathrm{HgAlC}$ was performed by two separate outside laboratories. One laboratory used the Dade Dimension technique, applying the turbidimetric inhibition immunoassay principle, where the total hemoglobin was based on a modification of the alkaline 
hematin reaction (McMillan, 2009). The percentage of total hemoglobin that was glycated was calculated and reported as $\% \mathrm{HgA} 1 \mathrm{C}$. The reference range is 4.8 to $6.0 \%$. The other laboratory performed $\mathrm{HgA1C}$ testing using the BioRad Variant II system, which used ion exchange highperformance liquid chromatography (HPLC) to determine percentage of glycated hemoglobin (Hinkle, 2009). The reference range is 4.4\% to 6.8\%. The American Diabetes Association (2010) recommends that laboratories use only Glycohemoglobin assay methods that have been approved by the National Glycohemoglobin Standardization Program. Both laboratories met these requirements and reported results in percentage of $\mathrm{HgA1C}$. While the laboratories reported different reference ranges, both laboratories reported values in percentage of $\mathrm{HgA1C}$. Hence, the difference in laboratory testing procedures was not clinically significant and would not affect percentage values or clinical decision making. This study collected the first $\mathrm{HgA} 1 \mathrm{C}$ available prior to May 2007, and the most recent $\mathrm{HgA1C}$ recorded on or before August 18, 2009. These data were recorded and analyzed as a continuous variable.

\section{Fasting Blood Glucose}

Fasting blood glucose measures blood glucose after a patient has not eaten for at least 8 hours. Fasting blood glucose was self-reported by the patient and recorded in the clinic visit note. All patients measure fasting blood glucose with a glucometer provided for home use by the free clinic. The meter then displays the level in milligrams per deciliter. This study collected the first fasting glucose available prior to May 2007 and the most recent fasting blood glucose recorded on or before August 18, 2009. These data were recorded and analyzed as a continuous variable.

\section{Serum Creatinine}

A serum creatinine test measures the amount of creatinine in the blood. The test was done to evaluate kidney function. Creatinine levels were drawn as part of routine diabetes care. In this 
clinic, the measurement of serum creatinine was performed by two separate outside laboratories. Both laboratories reported creatinine in milligrams per deciliter. The reference range for both laboratories is $0.5-1.2$ milligrams per deciliter. This study collected the first serum creatinine available prior to May 2007, and the most recent serum creatinine recorded on or before August 18, 2009. These data were recorded and analyzed as a continuous variable.

\section{Serum Lipid levels}

Serum lipid levels are considered to be any major lipid in the circulation. Serum lipid levels were routinely collected and reported as total cholesterol, high density lipoproteins (HDL), low density lipoproteins (LDL), and triglycerides (TG). Serum lipid levels were drawn as part of routine diabetes care. In this clinic the measurement of serum lipid was performed by two separate outside laboratories. Both laboratories reported serum lipids in milligrams per deciliter. The normal reference range was equivalent in both laboratories. This study collected the first serum lipids available prior to May 2007, and the most recent serum lipids recorded on or before August 18, 2009. These data were recorded and analyzed as four separate continuous variables.

\section{Urine Microalbumin}

Often urine microalbumin can be an earlier sign of potential kidney disease than serum creatinine. A urine microalbumin test measures the amount of albumin in the urine. The test was done to evaluate kidney function. Urine microalbumin was collected as part of routine diabetes care. In this clinic the measurement of urine microalbumin was performed by two separate outside laboratories. Both laboratories reported urine microalbumin in milligrams per deciliter. The normal reference range was equivalent in both laboratories. This study collected the first urine microalbumin available prior to May 2007, and the most recent urine microalbumin 
recorded on or before August 18, 2009. These data were recorded and analyzed as a continuous variable.

\section{Blood Pressure}

Blood pressure was obtained routinely and recorded in the chart at the beginning of each clinic visit. Blood pressure was measured and recorded as systolic over diastolic millimeters of mercury. The clinic used an automated blood pressure cuff. This study collected the first blood pressure available prior after May 2007, and the most recent blood pressure recorded on or before August 18, 2009. These data were recorded and analyzed as two separate continuous variables.

\section{Patient Characteristics}

Patient characteristics collected were: age, gender, ethnicity, marital status, duration of diabetes, education level, distance in miles from residence to clinic, depression score, and comorbidities.

Age

Age was collected from the chart from date of birth. Age was recorded at the age of the first visit within the time frame for the study. These data were recorded and analyzed as a continuous variable.

\section{Gender}

Gender was collected from the chart, and recorded as a dichotomous variable, either male or female. 


\section{Ethnicity}

Ethnicity, recorded upon establishment of care at the clinic, was collected from the chart. Ethnicity was collected by patient self-report. Ethnicity was recorded in the following categories: White, African-American, Asian, Hispanic, Native American, and other.

\section{Marital status}

Marital status was collected from the chart. Marital status is asked on the initial visit and was reassessed every year. Marital status was collected by patient self-report. The most recently recorded marital status was collected in the following categories: single, married, divorced, separated, widowed, significant other.

\section{Duration of diabetes}

Duration of diabetes was collected by chart review using patient self report data. Subjects are asked to report the number of years that they have had diabetes during clinic visits. The duration of diabetes was recorded from the beginning of the study period. Duration of diabetes was recorded as a continuous variable.

\section{Educational Level}

Education was collected by chart review. Education was recorded in the chart upon initial visit to the clinic. Education was recorded from the beginning of the study period in the following categories: less than high school, graduated high school, some college, college graduate, master's degree, doctorate, GED.

Distance in miles from residence to clinic

Home address was recorded in the chart at the initial visit and is verified every visit. Miles from residence to the clinic was calculated with Yahoo Map Quest using the clinic address 
and the patient address. Home address was not kept in any study data file. Miles from residence to clinic was analyzed as a continuous variable.

\section{Depression Score}

Depression score was collected upon initial visit, using The Center for Epidemiologic Studies Depression Scale (CES-D) (Deeb-Sossa, 2003). The CES-D is a commonly used screening test for determining depression quotient. While the tool is not diagnostic of clinical depression, it has been used in the past as an indicator of depression. The CES-D was filled out by the patient and can be completed in less than five minutes at the first visit prior to receiving care at the free clinic. While the CES-D was to be filled out yearly, it is not commonly updated at the free clinic. Hence, it was not be measured as an outcome of care but rather as a patient characteristic. The CES-D measured depressive feelings and behaviors during the past week. Each question was scored using a range of zero to three points. A score of less than 15 indicated no or few depressive feelings and behaviors during the past week. A score of 15-21 indicated mild to moderate depression. A score of over 21 indicated the possibility of major depression. Depression score was collected and recorded as a categorical variable.

\section{Co-Morbidities}

An ongoing list of active and prior medical conditions was kept on the medical chart. For the purposes of this study, a co-morbidity was the diagnosis of all other chronic diseases an individual patient might have other than diabetes. The data were recorded as a continuous variable, reflecting the total number of co-morbidities the patient has and as a dichotomous variable as yes/no for each of the following co-morbidities: hypertension, kidney disease, hyperlipidemia, heart disease, depression, obesity, kidney disease, pain, eye disease, neuropathy, and frequent infections. In order to further investigate the impact of co-morbid conditions a 
predictive index such as the Charlson co-morbidity scale was considered for use. . However, due to the retrospective nature of this study, all data were not available to make use of the Charlson co-morbidity index.

\section{Sample}

This retrospective study was conducted at a free clinic in West Virginia, using chart review of a convenience sample of patients who met the inclusion criteria. This study was approved by the Institutional Review Board of West Virginia University. The inclusion criteria are: 1) age $\geq 18$ years, 2) diagnosis of diabetes, 3) uninsured and received care at a free clinic during the study period. The only specific exclusion criteria would be an accidental charting of diabetes where no diabetes exists. Two independent groups were studied, those who attended DGMVs and those who did not attend DGMVs. To achieve a power of 0.8 considering a medium effect size to detect a difference in means between two independent groups, a sample of 51 charts in each group was required (Calculated with $G^{*}$ Power 3). The significance level of $\mathrm{p}=0.05$ was used.

\section{Data Collection}

A registry of all persons with diabetes who are patients was kept by the free clinic. The registry was reviewed by the research and clinic staff. All persons with diabetes who received care from May 2007, when DGMVs were started at the clinic, to August 18, 2009 were identified. The clinic underwent a change to electronic medical records throughout the year 2008. Hence, some data were obtained from previous paper medical records and current electronic medical records. The medical records of all persons with diabetes within the study time frame were pulled from the medical record room by the staff at the clinic. If the data were entirely electronic, the electronic medical record was reviewed. No patient identifiers were 
collected. No information was able to be linked back to the subject. The data were extracted from the paper medical record or electronic medical record by a medical assistant at the clinic. The medical assistant was educated by the investigator on data collection procedures. The medical assistant was provided with a training manual to keep in the clinic as a reference guide. The investigator was available via phone or e-mail for additional questions. Ten percent of the charts reviewed by the medical assistant over the first week of data collection were also reviewed by the investigator. An inter-rater reliability of $95 \%$ accuracy between the data collected by medical assistant and the data collected by the investigator was set and met. Identified charts were reviewed by the investigator or medical assistant in a private setting in the clinic. Biophysical outcomes of care were collected from the chart and recorded from two separate time periods. Biophysical outcomes for patients who did not participate in DGMVs were collected as reported in the chart during or after May 2007 and then again after one year. Biophysical outcomes of care were collected from the chart of patients who did participate in DGMVs prior to the initial DGMV and then again in one year. Data were entered into Microsoft Access for ease of use for the medical assistant collecting the data and then were converted into Statistical Package of Social Sciences (SPSS), version 18 for analysis of the data. Data was stored on a USB travel drive which was and continues to be password protected. The USB travel drive was and will continue to be kept in a locked office at the clinic or the locked office of the investigator when not in use.

\section{Analysis Plan}

Prior to analysis, the data were cleaned, looking for outliers or impossible values. This was accomplished by running frequencies and descriptive statistics and visually scanning for missing data and for patterns of missing data. Any variable item that had missing data such that it decreased power, or missing data with any identifiable pattern was not analyzed. 
Aim 1: To describe the characteristics of uninsured persons with diabetes who are cared for in a free clinic.

To describe the characteristics of the study sample descriptive statistics was used. The categorical variables gender, ethnicity, marital status, education, and type of co-morbidities were analyzed using frequencies and frequency tables and were reported as percentages. The continuous variables age, duration of diabetes, number of co-morbidities, and miles from the clinic were analyzed using mean, median and standard deviation. Aim2: To describe the biophysical outcomes of care in uninsured persons with diabetes who are cared for in a free clinic.

To describe the biophysical outcomes of the study sample, descriptive statistics were used. The continuous variables body weight, BMI, fasting blood glucose, $\mathrm{HgA1C}$, creatinine, lipids, blood pressure, and microalbumin were analyzed using mean, median and standard deviation.

Aim 3: To compare biophysical outcomes of care in persons with diabetes before attending DGMVs and again one year after attending DGMVs.

To compare biophysical outcomes of care in persons with diabetes before attending DGMVs and again one year after attending DGMVs paired t-tests were used. Differences in means of body weight, BMI, $\mathrm{HgA1C}$, fasting blood glucose, serum creatinine, serum lipids, urine microalbumin, systolic blood pressure, and diastolic blood pressure before attendance and DGMVs were compared to means at one year after attending DGMVs. The level of measurement for these variables was at the interval/ratio level. The data were obtained from a representative 
sample from the population. Tests for the assumptions necessary for the use of parametric statistics, such as normal distribution and homogeneity of variance, were performed. If after preliminary data analysis was performed, violation of assumptions was recognized, Wilcoxon Signed Rank test was used. If no assumptions were violated, a paired t-test was used. A value of $\mathrm{p}=0.05$ was used to determine significance of the findings.

Aim 4: To compare biophysical outcomes of care in uninsured persons with diabetes who receive usual care in a free clinic at baseline and after one year.

To compare means of biophysical outcomes of care in uninsured persons with diabetes who receive care in a free clinic and again after one year, paired t-tests were used to look at the difference in means of body weight, $\mathrm{BMI}, \mathrm{HgA1C}$, fasting blood glucose, serum creatinine, serum lipids, urine microalbumin, systolic blood pressure, and diastolic blood pressure. The level of measurement for these variables was at the interval/ratio level. The data were obtained from a representative sample from the population. Tests for the assumptions necessary for the use of parametric statistics, such as normal distribution of differences were performed. If after preliminary data analysis was performed, violation of assumptions was recognized, Wilcoxon Signed Rank test was used. If no assumptions were violated, a paired t-test was be used. A value of $p=0.05$ was used to determine significance of the findings.

Aim 5: To analyze the differences in biophysical outcomes in persons with diabetes who attend DGMVs versus those who receive usual care in a free clinic at base line and then again after one year.

To analyze the differences in biophysical outcomes in persons with diabetes who attend DGMVs versus those who receive usual care in a free clinic after one year, independent t-tests were used to compare means for body weight, BMI, $\mathrm{HgA1C}$, fasting blood glucose, serum 
creatinine, serum lipids, urine microalbumin, systolic blood pressure, and diastolic blood pressure. The level of measurement for these variables was at the interval/ratio level. The data were obtained from a representative sample from the population. The data were independent of one another. Tests for the assumptions necessary for the use of parametric statistics, such as normal distribution of each population and homogeneity of variance, were performed. If after preliminary data analysis was performed, violation of assumptions was recognized, MannWhitney $\mathrm{U}$ test was used. If no assumptions were violated, an independent-samples t-test was used. A value of $\mathrm{p}=0.05$ was used to determine significance of the findings.

Aim 6: To analyze the differences in characteristics of uninsured persons with diabetes who are attendees of DGMVs versus those who receive usual care.

To analyze the differences in characteristics of uninsured persons with diabetes who attend DGMVs versus those who receive usual care in a free clinic after one year group, means were calculated for each patient characteristic. Chi-square tests were used to look for differences in the categorical variables of gender, ethnicity, marital status, education, and type of comorbidities in patients who attended DGMV and patients who received usual care. Independent t-tests were used to compare means for age, duration of DM, miles from clinic, and number of co-morbidities. An assumption for use of the chi-square analysis is that the expected count in each category is greater than 5 . If this assumption is violated, the categories of the categorical variables were collapsed until the assumption was met. A value of $p=0.05$ was used to determine significance of the findings.

Aim 7: To explore the impact of dose of DGMVs on biophysical outcomes of care in uninsured persons with diabetes who receive care in a free clinic after one year. 
To explore the relationship between the dosage of DGMVs and biophysical outcomes of care in uninsured persons with diabetes who receive care in a free clinic after one year, dose was collected a continuous variable on a scale from one to six. Correlations were performed with the outcomes of body weight, BMI, $\mathrm{HgA1C}$, fasting blood glucose, serum creatinine, serum lipids, urine microalbumin, systolic blood pressure, and diastolic blood pressure at one year for those patients who attended DGMVs. Before performing a correlation analysis, a scatterplot was generated to check for violation of the assumptions of linearity. Each continuous variable was tested for normality. If assumptions of parametric testing were not violated Pearson $\mathrm{r}$ was used for analysis. If assumptions were violated Spearman rho was used for analysis. A value of $\mathrm{p}=0.05$ was used to determine significance of the findings.

\section{Data Fidelity}

Due to the retrospective nature of the study, intervention fidelity is difficult to control. However, group visits at the clinic were conducted by one diabetes educator, one nurse practitioner and one medical assistant. The group intervention was held in the same education room in the clinic and the healthcare portion of the visit was delivered in one of six very similar exam rooms. The educational content of all of the group visits were derived from one of six educational sessions developed for a standardized curriculum. The delivery format was consistent for each DGMV: instruction, questions, answers, goal setting and then individual patient health examinations. 


\section{CHAPTER 4: RESULTS}

The purpose of this study was to examine the relationship between attendance at Diabetes Group Medical Visits (DGMV) and biophysical outcomes of care in uninsured persons with diabetes who receive care in a free clinic. This chapter discusses the results of the data analysis conducted after the retrospective chart review was completed. This chapter will be presented in the following manner: data collection, data treatment, sample, results, and summary.

\section{Data Collection}

The following paragraph describes how the data for this study were obtained. The free clinic keeps a registry of all persons with diabetes. The registry was reviewed by the researcher and clinic staff. The charts of all persons with diabetes who received care from May 2007, when DGMVs were started at the clinic to August 18, 2009, were identified. The clinic underwent a change to electronic medical records throughout the year 2008. Hence, data were obtained from previous paper medical records and the current electronic medical records. No patient identifiers were collected. No information is able to be linked back to the patient. A medical assistant at the clinic extracted the data from the paper medical record and/or electronic medical record. The investigator educated the medical assistant about data collection procedures. Additionally, the medical assistant was provided with a data collection training manual, which was kept in the clinic as a reference guide. Ten percent of the charts reviewed by the medical assistant were also reviewed by the investigator. An inter-rater reliability of $95 \%$ accuracy between the data collected by medical assistant and the data collected by the investigator was set and met. Identified charts were reviewed by the investigator or medical assistant in a private setting in the clinic. Biophysical outcomes of care were collected from the chart and recorded for two separate time periods. Biophysical outcomes for patients who did not participate in DGMVs were 
collected as reported in the chart during or after May 2007 and then again after one year.

Biophysical outcomes of care were collected from the chart of patients who did participate in DGMVs prior to the initial DGMV and then again in one year.

\section{Sample}

This retrospective study was conducted with a convenience sample of patients at a free clinic in West Virginia. The data were obtained by reviewing the charts of all persons with diabetes who received care from May 2007 to August 18, 2009. Two independent groups were studied, those who attended DGMVs and those who received usual care. There were a total of 111 patients who met the inclusion criteria. There were 53 participants who attended DGMVs and 58 participants who received usual care. This study was approved by the Institutional Review Board of West Virginia University.

\section{Data Treatment}

Data were entered into Microsoft Access and then were transferred into the Statistical Package of Social Sciences (SPSS), version 18 for analysis of the data. Data were stored on a USB travel drive which was password protected. The USB travel drive was and will continue to be kept in a locked office of the investigator when not in use.

Prior to analysis, data were cleaned, looking for outliers or impossible values. Looking for outliers was accomplished by analyzing frequencies and descriptive statistics and visually scanning the data for impossible values, missing data and patterns of missing data. Duration of diabetes had $90.1 \%$ missing data. Hence, duration of diabetes was excluded from further analysis. The variable "depression score" had 27.9 percent $(\mathrm{N}=22)$ missing data for the total sample. The patients who attended group visits had depression scores missing from 11 cases (9.9\%). The patients who received usual care had depression scores missing from 20 cases 
(18\%). For the purpose of this study, depression score was analyzed as a characteristic to describe the groups prior to intervention, not to compare the effectiveness of the intervention. The missing depression data will be noted as a limitation of the study. Microalbumin had 27\% $(\mathrm{N}=30)$ missing data at time one and $29.7 \%(\mathrm{~N}=33)$ missing data at time two from the entire sample. Patients who attended DGMVs had no missing data in microalbumin at time one and 3 cases $(2.7 \%)$ were missing microalbumin data at time two. Patients who received usual care were missing microalbumin in 30 cases (27\%) at time one and 30 cases (27\%) at time two. The observed power for an independent t-test to compare microalbumin data in those who attended DGMVs versus usual care at time one and time two $(\mathrm{N}=78)$ is 0.616 using a significance level $(\mathrm{p}=0.05)$, and a medium effect size. The observed power for microalbumin data may not be enough detect a significant difference in microalbumin in those who attended DGMVs versus usual care at time one and time two. The large amount of missing data for microalbumin for the patients who received usual care will be listed as a limitation of the study. Patients who received usual care had 13 cases $(11.7 \%)$ of lipid results missing at time two. The observed power for an independent t-test to compare lipid data in those who attended DGMVs versus usual care at time one and time two $(\mathrm{N}=98)$ is 0.825 using a significance level $\mathrm{p}=0.05$, and a medium effect size. The observed power for an independent t-test is enough to show a significant difference, if one exists, in lipid data in those who attended DGMVs versus usual care at time two. No other variable had greater than $10 \%$ missing data.

\section{Statistical Assumptions}

The following paragraphs describe the statistical assumptions used for this study. To compare biophysical outcomes of care in persons with diabetes before attending DGMVs and again one year after attending DGMVs, paired t-tests were used. Paired t-tests were also used to 
compare means of biophysical outcomes of care in uninsured persons with diabetes who receive usual care in a free clinic and again after one year. Chi-square tests were used to look for differences in the categorical variables between patients who attended DGMVs and patients who received usual care. To examine dose of DGMVs, correlations were performed with biophysical outcomes at one year for those patients who attended DGMVs.

Normal distribution and random samples are assumptions for both a paired t-test and an independent t-test. Q-Q plots were used to evaluate normal distribution. If Q-Q plots showed a normal distribution of the data, paired t-tests or independent t-tests were considered appropriate to analyze differences in biophysical outcomes. If Q-Q plots showed a non-normal distribution of the data, either the non-parametric alternative test was used or the Central Limit Theorem was applied. The non-parametric alternative test for a paired t-test is the Wilcoxon Signed Rank test. The non-parametric alternative test for an independent t-test is the Mann-Whitney $\mathrm{U}$ test. The Central Limit Theorem states that as the size of a sample of independent observations approaches infinity, the sampling distribution of the sample mean approaches a normal distribution. Hence, if $\mathrm{n}$ is large enough, typically greater than 30 , the Central Limit Theorem can be applied (http://www.encyclopedia.com/doc/1G2-3045300301.html, 2008). Eta squared is the statistic used to measure the strength of relationship between two variables for a paired-samples t-test. Independent $\mathrm{t}$-tests have additional assumptions. To use the independent t-test the data must be independent of each other. Another assumption of an independent t-test is that the population variances are equal. In this study, Levene's test was used to evaluate the assumption of equal variances. If the Levene's test $\mathrm{p}$ value was larger than .05 , equal variances were assumed. If the Levene's test $\mathrm{p}$ value was less than .05 , the results were interpreted using the $t$ value for the equal variances not assumed test. 
An assumption for use of the chi-square analysis is that the expected count in each category is greater than 5. If this assumption was violated, the categories of the categorical variables were collapsed until the assumption was met. If the assumption of an expected count in each category of greater than 5 could not be met, the data were not analyzed.

Before performing a correlation analysis, scatterplots were generated to check for violation of the assumptions of linearity. Each continuous variable was tested for normality. If assumptions of parametric testing were not violated, Pearson $r$ was used for analysis. If assumptions were violated Spearman rho was used for analysis.

\section{Results}

The results will be presented according to the aims of the study. The patient characteristics will be presented followed by the biophysical outcomes of the sample. Then the comparison of characteristics and biophysical outcomes between each group will be presented. Finally, the impact of dose of DGMVs on biophysical outcomes of care will be explored. Each aim is listed, followed by results and data charts of each result. A value of $p=0.05$ was used to determine significance of all findings.

Aim 1: To describe the characteristics of uninsured persons with diabetes who are cared for in a free clinic.

To describe the characteristics of the study sample, descriptive statistics were used. The categorical variables gender, ethnicity, marital status, education, and type of co-morbidities were analyzed using frequencies. The majority of the patients were female, white, married, and had a high school education or less (See Table 1). Sixty-five percent of the patients were obese and the majority of the patients had hypertension (84\%) and hyperlipidemia (64\%). The continuous variables age, number of co-morbidities, and miles from the subject's home to the clinic were 
analyzed using mean and standard deviation. The mean age of the subjects in the study was 48 years. The mean number of co-morbidities was 5 and the mean distance from the patient's home to the clinic was 21 miles (See Table 2). 
Table 1: Sample Characteristics Categorical Variables

Demographic

Variable

Percent

Gender

Male

29

26.1

Female

82

73.9

\section{Ethnicity}

White

107

95.5

African American

4

3.6

Hispanic

1

0.9

\section{Marital Status}

Single

13.5

Married

56

50.5

Divorced

24

21.6

Separated

Widowed

\section{Education Level}

Less Than High School

Graduated High School

$\begin{array}{rr}27 & 24.3 \\ 44 & 39.6 \\ 20 & 18.0 \\ 1 & .9 \\ 15 & 13.5\end{array}$

Some College

College Graduate

\section{Co-Morbidity}

HTN

Kidney Disease

Hyperlipidemia

Heart Disease

Depression

Obesity

Pain

Neuropathy

Frequent Infections
84

13

64

18

39

73

16

9

5
75.7

11.7

57.7

16.2

35.1

65.8

14.4

8.1

4.5 
Table 2: Sample Characteristics Continuous Variables

\begin{tabular}{lrrr}
\hline Demographic & Mean & Range & SD \\
\hline Age in years & 48 & $21-64$ & 10.8 \\
Total number of co-morbidities & 5 & $0-11$ & 1.3 \\
Distance from clinic in miles & 21 & $0.7-124$ & 20.4 \\
\hline
\end{tabular}

Aim 2: To describe the biophysical outcomes of care in uninsured persons with diabetes who are cared for in a free clinic.

To describe the biophysical outcomes at time one of the study sample, descriptive statistics were used. The continuous variables body weight, BMI, fasting blood glucose, $\mathrm{HgAlC}$, creatinine, lipids, blood pressure, and microalbumin were analyzed using mean and standard deviation (See Table 3). It is noted that there is a large standard deviation in urine microalbumin. Because these microalbumin numbers are known to be actual patient results and not aberrant data entry mistakes, the values were included in the data evaluation. The mean body weight for subjects in this study was 226.5 pounds and the mean BMI of the subjects was 37.6. The mean $\mathrm{HgA} 1 \mathrm{C}$ in this population was $8 \%$. The mean for urine microalbumin was $52.9 \mathrm{mg}$. The mean LDL cholesterol level of this sample was $104.6 \mathrm{mg} / \mathrm{dl}$. The mean HDL cholesterol of this sample was $40.4 \mathrm{mg} / \mathrm{dl}$. The mean triglyceride level of this population was $198.6 \mathrm{mg} / \mathrm{dl}$.

Table 3: Biophysical Outcomes of Care

\begin{tabular}{lrrrr}
\hline $\begin{array}{l}\text { Biophysical } \\
\text { Outcomes }\end{array}$ & N & Mean & Range & SD \\
\hline Body Weight (lbs) & 111 & 226.59 & $121-400$ & 52.71 \\
BMI (kg/mg2) & 111 & 37.60 & $21.5-58.7$ & 28.48 \\
HgA1C (\%) & 110 & 8.09 & $5-13.6$ & 1.94 \\
Blood Glucose (mg/dl) & 111 & 183.94 & $51-568$ & 89.55 \\
Creatinine (mmol/l) & 111 & 0.93 & $.44-4.2$ & 0.45 \\
Microalbumin (mg/mmol) & 81 & 52.95 & $.20-1120.8$ & 157.96 \\
Systolic (mmHg) & 111 & 129.50 & $90-190$ & 19.65 \\
Diastolic (mmHg) & 111 & 80.41 & $59-121$ & 13.15 \\
Total Cholesterol (mg/dl) & 108 & 188.82 & $88-337$ & 45.80 \\
HDL (mg/dl) & 107 & 40.40 & $17-83$ & 10.63 \\
LDL (mg/dl) & 104 & 104.65 & $5-201$ & 40.44 \\
Triglycerides (mg/dl) & 103 & 198.67 & $36-1156$ & 169.17 \\
\hline
\end{tabular}


Aim 3: To compare biophysical outcomes of care in persons with diabetes before attending DGMVs and again one year after attending DGMVs.

To compare biophysical outcomes of care in persons with diabetes before attending DGMVs and again one year after attending DGMVs, paired t-tests were used. The sample in this study is not random. This retrospective study was conducted on a convenience sample of patients at a free clinic in West Virginia. However, paired t-tests are commonly used in retrospective studies. Q-Q plots were analyzed on the differences between time one and time two for the following biophysical outcomes: body weight, BMI, $\mathrm{HgA1C}$, fasting blood glucose, serum creatinine, serum lipids, urine microalbumin, systolic blood pressure, and diastolic blood pressure. The data did not meet the assumption of normality for the following biophysical outcomes: body weight, BMI, creatinine, microalbumin, and triglycerides (See Appendix A). However, since the sample size was large, the results of parametric testing are still valid due to the Central Limit Theorem. Hence paired-samples t-tests were used to evaluate the impact of DGMVs on patients' biophysical outcomes of care.

Means of body weight, BMI, HgA1C, fasting blood glucose, serum creatinine, serum lipids, urine microalbumin, systolic blood pressure, and diastolic blood pressure before attendance at DGMVs were compared to means at one year after attending DGMVs. There was a statistically significant decrease in systolic blood pressure from time one $(\mathrm{M}=132.32, \mathrm{SD}=18.31)$ to time two $(\mathrm{M}=126.83, \mathrm{SD}=18.31), t(52)=2.18,(\mathrm{p}=0.03)$. The mean decrease in systolic blood pressure from time one to time two was $5.49 \mathrm{~mm} / \mathrm{Hg}$ with a $95 \%$ confidence interval ranging from 0.443 to 10.539 , a range of values for the estimated population parameter. The eta squared statistic (.08) indicated a moderate effect size. No other significant impact on biophysical 
outcomes of care in persons with diabetes before attending DGMVs and again after attending DGMVs was noted (See Table 4). 
Table 4: Biophysical Outcomes of Care Before and After DGMVs

\begin{tabular}{|c|c|c|c|c|c|c|}
\hline Biophysical Outcome & $\mathbf{N}$ & Mean & SD & df & $t$ & $p$ \\
\hline Body Weight Time One (lbs) & 53 & 232.11 & \multirow{2}{*}{17.50} & \multirow{2}{*}{52.00} & \multirow[t]{2}{*}{-1.77} & \multirow[t]{2}{*}{0.08} \\
\hline Body Weight Time Two (lbs) & 53 & 236.38 & & & & \\
\hline BMI Time One (kg/mg2) & 53 & 38.21 & \multirow{2}{*}{2.78} & \multirow{2}{*}{52.00} & \multirow{2}{*}{-0.095} & \multirow{2}{*}{0.37} \\
\hline BMI Time Two (kg/mg2) & 53 & 38.58 & & & & \\
\hline HgA1C Time One (\%) & 53 & 8.65 & \multirow{2}{*}{1.99} & \multirow{2}{*}{52.00} & \multirow{2}{*}{-0.16} & \multirow{2}{*}{0.87} \\
\hline HgA1C Time Two (\%) & 53 & 8.69 & & & & \\
\hline Blood Glucose Time One (mg/dl) & 53 & 194.17 & \multirow{3}{*}{99.14} & \multirow{3}{*}{52.00} & \multirow{3}{*}{-0.42} & \multirow{3}{*}{0.67} \\
\hline & & & & & & \\
\hline Blood Glucose Time Two (mg/dl) & 53 & 199.92 & & & & \\
\hline Creatinine Time One $(\mathrm{mg} / \mathrm{mmol})$ & 51 & 0.89 & \multirow{2}{*}{0.27} & \multirow{2}{*}{50.00} & \multirow{2}{*}{-1.05} & \multirow{2}{*}{0.30} \\
\hline Creatinine Time Two $(\mathrm{mg} / \mathrm{mmol})$ & 51 & 0.93 & & & & \\
\hline Microalbumin Time One (mg//mmol) & 50 & 59.36 & \multirow[t]{2}{*}{165.94} & \multirow[t]{2}{*}{49.00} & \multirow[t]{2}{*}{1.52} & \multirow[t]{2}{*}{0.13} \\
\hline Microalbumin Time Two (mg//mmol) & 50 & 23.60 & & & & \\
\hline Systolic Time One (mgHg) & 53 & 132.32 & \multirow[t]{2}{*}{18.31} & \multirow[t]{2}{*}{52.00} & \multirow[t]{2}{*}{2.18} & \multirow[t]{2}{*}{0.03} \\
\hline Systolic Time Two (mgHg) & 53 & 126.83 & & & & \\
\hline Diastolic Time One (mgHg) & 53 & 81.92 & \multirow[t]{2}{*}{12.38} & \multirow[t]{2}{*}{52.00} & \multirow[t]{2}{*}{1.22} & \multirow[t]{2}{*}{0.23} \\
\hline Diastolic Time Two (mgHg) & 53 & 79.85 & & & & \\
\hline Total Cholesterol Time One (mg/dl) & 52 & 192.67 & \multirow[b]{2}{*}{48.53} & & & \\
\hline Total Cholesterol Time Two (mo/dl) & 52 & 18963 & & 51.00 & 0.45 & 0.65 \\
\hline HDL Time One (mg/dl) & 52 & 40.38 & 8.93 & 51.00 & -0.95 & 0.35 \\
\hline HDL Time Two (mg/dl) & 52 & 41.56 & & & & \\
\hline LDL Time One (mg/dl) & 48 & 100.73 & 44.91 & 47.00 & 0.95 & 0.35 \\
\hline LDL Time Two (mg/dl) & 48 & 94.58 & & & & \\
\hline Triglycerides Time One (mg/dl) & 49 & 236.31 & 192.56 & 48.00 & 0.72 & 0.48 \\
\hline Triglycerides Time Two (mg/dl) & 49 & 216.63 & & & & \\
\hline
\end{tabular}


Aim 4: To compare biophysical outcomes of care in uninsured persons with diabetes who receive usual care in a free clinic at baseline and after one year.

To compare means of biophysical outcomes of care in uninsured persons with diabetes who receive usual care in a free clinic and again after one year paired t-tests were used. Again, the sample in this retrospective study was not random. However, paired t-tests are commonly used in retrospective studies. Q-Q plots were analyzed on the differences between time one and time two for the following biophysical outcomes: body weight, BMI, $\mathrm{HgA1C}$, fasting blood glucose, serum creatinine, serum lipids, urine microalbumin, systolic blood pressure, and diastolic blood pressure. For those who receive usual care, the data do not meet the assumption of normality for the following biophysical outcomes: body weight, $\mathrm{HgA1C}$, BMI, blood glucose, creatinine, microalbumin, diastolic blood pressure, LDL, and triglycerides (See Appendix B). However, due to the Central Limit Theorem, parametric testing is still valid for all biophysical outcomes except microalbumin. The Central Limit Theorem cannot be applied for the microalbumin outcome, since it contains less than 30 cases. Hence paired-samples t-tests were used to evaluate the impact of usual care on patients for all biophysical outcomes of care except microalbumin. Wilcoxon Signed Rank test was used to evaluate the impact of usual care on microalbumin.

Means of body weight, BMI, HgA1C, fasting blood glucose, serum creatinine, serum lipids, urine microalbumin, systolic blood pressure, and diastolic blood pressure in patients who receive usual care at baseline and again after one year were compared. There was no significant difference between biophysical outcomes of care from time one to time two noted (See Table 5). 
Table 5: Biophysical Outcomes of Care for Usual Care Patients at Baseline and After One Year

\begin{tabular}{|c|c|c|c|c|c|c|}
\hline Biophysical Outcome & $\mathbf{N}$ & Mean & SD & df & $t$ & $p$ \\
\hline Body Weight Time One (lbs) & 58 & 221.55 & 57.25 & \multirow{2}{*}{57} & \multirow{2}{*}{0.21} & \multirow{2}{*}{.836} \\
\hline Body Weight Time Two (lbs) & 58 & 221.17 & 53.27 & & & \\
\hline BMI Time One (kg/mg2) & 58 & 37.08 & 8.83 & \multirow{2}{*}{57} & \multirow{2}{*}{0.33} & \multirow{2}{*}{.745} \\
\hline BMI Time Two (kg/mg2) & 58 & 36.98 & 8.10 & & & \\
\hline HgA1C Time One (\%) & 52 & 7.52 & 1.66 & \multirow{2}{*}{51} & \multirow{2}{*}{0.13} & \multirow{2}{*}{.896} \\
\hline HgA1C Time Two (\%) & 52 & 7.49 & 1.55 & & & \\
\hline Blood Glucose Time One (mg/dl) & 58 & 174.59 & 86.82 & \multirow[b]{2}{*}{57} & \multirow[b]{2}{*}{-0.04} & \multirow[b]{2}{*}{.97(} \\
\hline Blood Glucose Time Two (mg/dl) & 58 & 175.05 & 83.95 & & & \\
\hline Creatinine Time One (mg/mmol) & 53 & 0.95 & 0.61 & \multirow{2}{*}{52} & \multirow{2}{*}{-0.60} & \multirow{2}{*}{.548} \\
\hline Creatinine Time Two (mg/mmol) & 53 & 0.97 & 0.55 & & & \\
\hline Microalbumin Time One (mg//mmol) & 19 & 64.33 & 140.36 & & \multirow{2}{*}{$-.558(\mathrm{z})$} & \multirow{2}{*}{.557} \\
\hline Microalbumin Time Two (mg//mmol) & 19 & 68.87 & 188.26 & & & \\
\hline Systolic Time One (mgHg) & 58 & 126.93 & 19.56 & \multirow{2}{*}{57} & \multirow{2}{*}{0.46} & \multirow{2}{*}{.647} \\
\hline Systolic Time Two (mgHg) & 58 & 125.88 & 13.76 & & & \\
\hline Diastolic Time One (mgHg) & 58 & 79.02 & 12.71 & \multirow{2}{*}{57} & \multirow{2}{*}{-0.89} & \multirow{2}{*}{.375} \\
\hline Diastolic Time Two (mgHg) & 58 & 80.64 & 11.82 & & & \\
\hline Total Cholesterol Time One (mg/dl) & 45 & 182.02 & 39.89 & \multirow[b]{2}{*}{44} & \multirow[b]{2}{*}{1.26} & \multirow[b]{2}{*}{.216} \\
\hline Total Cholesterol Time Two (mg/dl) & 45 & 175.87 & 33.87 & & & \\
\hline HDL Time One (mg/dl) & 45 & 40.89 & 10.55 & \multirow[t]{2}{*}{44} & \multirow[t]{2}{*}{-0.94} & \multirow[t]{2}{*}{.351} \\
\hline HDL Time Two (mg/dl) & 45 & 41.80 & 10.75 & & & \\
\hline LDL Time One (mg/dl) & 42 & 105.50 & 32.42 & \multirow[t]{2}{*}{41} & \multirow[t]{2}{*}{1.49} & \multirow[t]{2}{*}{.145} \\
\hline LDL Time Two (mg/dl) & 42 & 97.86 & 30.75 & & & \\
\hline Triglycerides Time One (mg/dl) & 42 & 171.33 & 125.86 & \multirow[t]{2}{*}{41} & 0.09 & .929 \\
\hline Triglycerides Time Two (mg/dl) & 42 & 169.57 & 105.21 & & & \\
\hline
\end{tabular}


Aim 5: To analyze the differences in biophysical outcomes in persons with diabetes who attend $D G M V$ s versus those who receive usual care in a free clinic at baseline and then again after one year.

Independent t-tests were used to compare means for body weight, BMI, $\mathrm{HgA1C}$, fasting blood glucose, serum creatinine, serum lipids, urine microalbumin, systolic blood pressure, and diastolic blood pressure between the usual care group and the DGMV group at baseline and then again after one year. Again, the sample in this retrospective study was not random. However, independent t-tests are commonly used in retrospective studies. Q-Q plots were analyzed to assess the distribution of each biophysical outcome for patients who attended DGMVs and for patients who received usual care separately at time one and at time two for the following biophysical outcomes: body weight, $\mathrm{BMI}, \mathrm{HgA1C}$, fasting blood glucose, serum creatinine, serum lipids, urine microalbumin, systolic blood pressure, and diastolic blood pressure (See Appendices C \& D). For those who attended DGMVs, the data does not meet the assumption of normality for the following biophysical outcomes at time one and time two: $\mathrm{HgA1C}$, blood glucose, creatinine, microalbumin, total cholesterol, HDL, LDL, and triglycerides. For those who received usual care the data does not meet the assumption of normality for the following biophysical outcomes at time one and time two: $\mathrm{HgA1C}$, blood glucose, creatinine, microalbumin, systolic blood pressure, diastolic blood pressure, total cholesterol, HDL, LDL, and triglycerides. However, the results of the parametric test are still valid due to the Central Limit Therom for the following variables in both groups: $\mathrm{HgA1C}$, blood glucose, creatinine, systolic blood pressure, diastolic blood pressure, total cholesterol, HDL, LDL, and triglycerides. The Central Limit Therom cannot be applied to compare the mean of microalbumin between groups at time one or at time two, because microalbumin contains less than 30 cases in the usual 
care group at both time one and time two. Hence, Mann-Whitney U test was used for the microalbumin comparisons. The homogeneity of variances assumption was violated for the biophysical outcomes of $\mathrm{HgA1C}$ at time one, $\mathrm{LDL}$ at time one, $\mathrm{HgA1C}$ at time two, systolic blood pressure at time two, and triglycerides at time two. Hence, the $t$ value for equal variances not assumed was used to interpret the results of the comparisons of $\mathrm{HgA1C}$ at time one, $\mathrm{LDL}$ at time one, $\mathrm{HgA1C}$ at time two, systolic blood pressure at time two, and triglycerides at time two. To determine if the patients who attended DGMVs had differing biophysical outcomes than the patients who received usual care prior to the DGMV intervention, means of body weight, BMI, $\mathrm{HgA1C}$, fasting blood glucose, serum creatinine, serum lipids, systolic blood pressure, and diastolic blood pressure in patients who attended DGMVs and those who received usual care at time one were compared. Due to the large amount of missing urine microalbumin data, the median of urine microalbumin in patients who attended DGMVs and those who received usual care at time one was used for comparison between the two groups. The patients who attended DGMVs had a higher HgA1C at baseline ( $\mathrm{p}=0.003$ ). There were no other significant differences in biophysical outcomes of care noted between the DGMV group and the usual care group at baseline, indicating that the groups were essentially similar prior to intervention (See Table 6).

To examine differences in biophysical outcomes one year after attending DGMVs or receiving usual care, means of body weight, $\mathrm{BMI}, \mathrm{HgA1C}$, fasting blood glucose, serum creatinine, serum lipids, systolic blood pressure, and diastolic blood pressure at time two were compared. Due to the large amount of missing urine microalbumin data, the medians of urine microalbumin in patients who attended DGMVs and those who received usual care at time two were compared. The patients who attended DGMVs continued to have a higher $\mathrm{HgA1C}$ after one 
year $(\mathrm{p}=0.001)$. There were no other significant differences in biophysical outcomes of care noted between the DGMV group and the usual care group at one year (See Table 7).

Although not statistically significant, the biophysical outcomes did change in the sample (See Table 8). Body weight increased in the DGMV group and BMI increased in both groups from time one to time two. Blood glucose increased in both groups from time one to time two. Creatinine increased in the DGMV group from time one to time two. Microalbumin decreased in both groups from time one to time two. Systolic blood pressure decreased in both groups. Diastolic blood pressure decreased in the DGMV group and increased in the usual care group from time one to time two. Total cholesterol decreased in both groups and HDL increased in both groups from time one to time two. LDL decreased in both groups from time one to time two. Triglycerides decreased in the DGMV group and increased in the usual care group from time one to time two. The only difference between the two groups at baseline was that the patients who attended DGMVs had a statistically higer $\mathrm{HgA1C}$. The patients who attended DGMVs continued to have a statistically significant higher $\mathrm{HgAlC}$ after one year. While the groups were equivalent except for a higher $\mathrm{HgAlC}$ in the group of patients who attended DGMVs, there were no other significant differences in biophysical outcomes of care noted at baseline or after one year. 
Table 6: Differences in Biophysical Outcomes in Those Who Attend DGMVs versus Usual Care at Baseline

\begin{tabular}{|c|c|c|c|c|c|}
\hline Biophysical Outcome & Mean & SD & df & $t$ & $p$ \\
\hline \multicolumn{6}{|l|}{ Body Weight (lbs) } \\
\hline Group Visits & 232.11 & 47.17 & 109 & 1.06 & .294 \\
\hline Usual care & 221.55 & 57.25 & & & \\
\hline \multicolumn{6}{|l|}{ BMI (kg/mg2) } \\
\hline Group Visits & 38.21 & 40.09 & 109 & 1.23 & .222 \\
\hline Usual care & 37.08 & 8.83 & & & \\
\hline \multicolumn{6}{|l|}{$\operatorname{HgA} 1 \mathrm{C}(\%)$} \\
\hline Group Visits & 8.65 & 2.09 & 108 & 3.02 & .003 \\
\hline Usual care & 7.57 & 1.64 & & & \\
\hline \multicolumn{6}{|l|}{ Blood Glucose(mg/dl) } \\
\hline Group Visits & 194.17 & 92.18 & 109 & 1.15 & .252 \\
\hline Usual care & 174.59 & 86.82 & & & \\
\hline \multicolumn{6}{|l|}{ Creatinine (mg/mmol) } \\
\hline Group Visits & 0.89 & 0.19 & 109 & -0.87 & .384 \\
\hline Usual care & 0.97 & 0.60 & & & \\
\hline \multicolumn{6}{|l|}{ Microalbumin (mg/mmol) } \\
\hline Group Visits & $3.2(M d)$ & & & $-1.053(\mathrm{z})$ & .292 \\
\hline Usual care & $6.8(M d)$ & & & & \\
\hline \multicolumn{6}{|l|}{ Systolic Blood Pressure (mgHg) } \\
\hline Group Visits & 132.32 & 19.54 & 109 & 1.45 & .150 \\
\hline Usual care & 126.93 & 19.56 & & & \\
\hline \multicolumn{6}{|l|}{ Diastolic Blood Pressure (mgHg) } \\
\hline Group Visits & 81.92 & 13.58 & 109 & 1.17 & .246 \\
\hline Usual care & 79.02 & 12.71 & & & \\
\hline \multicolumn{6}{|l|}{ Total Cholesterol (mg/dl) } \\
\hline Group Visits & 192.72 & 50.05 & 106 & 0.87 & .388 \\
\hline Usual care & 185.07 & 41.41 & & & \\
\hline \multicolumn{6}{|l|}{ HDL (mg/dl) } \\
\hline Group Visits & 40.38 & 10.65 & 105 & -0.02 & .981 \\
\hline Usual care & 40.43 & 10.71 & & & \\
\hline \multicolumn{6}{|l|}{ LDL (mg/dl) } \\
\hline Group Visits & 100.42 & 45.22 & 102 & -1.07 & .288 \\
\hline Usual care & 108.88 & 34.96 & & & \\
\hline \multicolumn{6}{|l|}{ Triglycerides (mg/dl) } \\
\hline Group Visits & 227.83 & 202.32 & 101 & 1.79 & .077 \\
\hline Usual care & 168.94 & 121.83 & & & \\
\hline
\end{tabular}


Table 7: Differences in Biophysical Outcomes in Those Who Attend DGMVs versus Usual Care at One Year

\begin{tabular}{|c|c|c|c|c|c|}
\hline Biophysical Outcome & Mean & SD & df & $t$ & $p$ \\
\hline \multicolumn{6}{|l|}{ Body Weight (lbs) } \\
\hline Group Visits & 236.38 & 52.69 & 109 & 1.51 & .134 \\
\hline Usual care & 221.17 & 53.27 & & & \\
\hline \multicolumn{6}{|l|}{ BMI (kg/mg2) } \\
\hline Group Visits & 38.58 & 8.48 & 109 & 1.01 & .313 \\
\hline Usual care & 36.98 & 8.10 & & & \\
\hline \multicolumn{6}{|l|}{$\mathrm{HgA} 1 \mathrm{C}(\%)$} \\
\hline Group Visits & 8.69 & 2.23 & 104 & 3.27 & .001 \\
\hline Usual care & 7.48 & 1.54 & & & \\
\hline \multicolumn{6}{|l|}{ Blood Glucose $(\mathrm{mg} / \mathrm{dl})$} \\
\hline Group Visits & 199.92 & 102.76 & 109 & 1.40 & .164 \\
\hline Usual care & 175.05 & 83.95 & & & \\
\hline \multicolumn{6}{|l|}{ Creatinine $(\mathrm{mg} / \mathrm{mmol})$} \\
\hline Group Visits & 0.93 & 0.28 & 102 & -0.45 & .653 \\
\hline Usual care & 0.97 & 0.55 & & & \\
\hline \multicolumn{6}{|l|}{ Microalbumin (mg/mmol) } \\
\hline Group Visits & $2.75(M d)$ & & 7 & -1.683 & .092 \\
\hline Usual care & $6.0(M d)$ & & & & \\
\hline \multicolumn{6}{|l|}{$\begin{array}{l}\text { Systolic Blood Pressure } \\
(\mathrm{mgHg})\end{array}$} \\
\hline Group Visits & 126.83 & 19.21 & 109 & 0.30 & .763 \\
\hline Usual care & 125.88 & 13.76 & & & \\
\hline \multicolumn{6}{|l|}{$\begin{array}{l}\text { Diastolic Blood Pressure } \\
(\mathrm{mgHg})\end{array}$} \\
\hline Group Visits & 79.85 & 11.74 & 109 & -0.35 & .725 \\
\hline Usual care & 80.64 & 11.82 & & & \\
\hline \multicolumn{6}{|l|}{ Total Cholesterol (mg/dl) } \\
\hline Group Visits & 189.63 & 55.55 & 96 & 1.38 & .172 \\
\hline Usual care & 176.63 & 33.89 & & & \\
\hline \multicolumn{6}{|l|}{$\mathrm{HDL}(\mathrm{mg} / \mathrm{dl})$} \\
\hline Group Visits & 41.56 & 11.84 & 96 & -0.14 & .892 \\
\hline Usual care & 41.87 & 10.64 & & & \\
\hline \multicolumn{6}{|l|}{$\mathrm{LDL}(\mathrm{mg} / \mathrm{dl})$} \\
\hline Group Visits & 95.41 & 38.16 & 90 & -0.06 & .955 \\
\hline Usual care & 95.84 & 33.15 & & & \\
\hline \multicolumn{6}{|l|}{ Triglycerides (mg/dl) } \\
\hline Group Visits & 216.30 & 151.48 & 91 & 1.61 & .111 \\
\hline Usual care & 172.02 & 105.19 & & & \\
\hline
\end{tabular}


Table 8: Changes in Biophysical Outcomes in Those Who Attend DGMVs versus Usual Care at Time Two

\begin{tabular}{|c|c|c|}
\hline Biophysical & DGMVs & Usual Care \\
\hline Body Weight (lbs) & $\Delta$ & - \\
\hline BMI (kg/mg2) & $\Delta$ & $\Delta$ \\
\hline $\operatorname{HgA1C}(\%)$ & - & - \\
\hline Blood Glucose $(\mathrm{mg} / \mathrm{dl})$ & $\Delta$ & $\Delta$ \\
\hline Creatinine $(\mathrm{mg} / \mathrm{mmol})$ & $\Delta$ & - \\
\hline Microalbumin (mg/mmol) & $\nabla$ & $\boldsymbol{\nabla}$ \\
\hline Systolic Blood Pressure & $\nabla$ & $\nabla$ \\
\hline Diastolic Blood Pressure & $\nabla$ & $\Delta$ \\
\hline Total Cholesterol (mg/dl) & $\nabla$ & $\Delta$ \\
\hline HDL (mg/dl) & $\Delta$ & $\Delta$ \\
\hline $\mathrm{LDL}(\mathrm{mg} / \mathrm{dl})$ & $\Delta$ & $\Delta$ \\
\hline Triglycerides (mg/dl) & $\nabla$ & $\Delta$ \\
\hline
\end{tabular}


Aim 6: To analyze the differences in characteristics of uninsured persons with diabetes who are attendees of DGMVs verses those who receive usual care.

Chi-square tests were used to look for differences in the categorical variables of gender, ethnicity, marital status, education, and type of co-morbidities between patients who attended DGMVs and patients who received usual care. An assumption for use of the chi-square analysis is that the expected count in each category is greater than 5. Education level and ethnicity violated the assumptions by having an expected count of less than 5 observations in several categories. Education level had only one participant that graduated from college and no participants graduated or attended graduate school. Hence, the categories were collapsed. The participant who graduated from college was included in the "some college" category and the "master's degree" and "doctoral degree" categories were removed. There were only five participants who reported being anything other than white. Hence, the categories were collapsed into white and non-white. The expected count was still less than 5 observations in ethnicity. Therefore, the characteristic of race/ethnicity was not analyzed. Marital status had an expected count of less than 5 observations in several categories. The marital status category was compressed into the categories married and not married. Any participant who was listed as single, divorced, separated, widowed was placed in the non-married category. Participants who were listed as married were placed in the married category.

Independent t-tests were used to compare means for the continuous characteristics of age, miles from clinic, and number of co-morbidities between patients who attended DGMVs and patients who received usual care. Again, the sample in this retrospective study was not random. However, independent t-tests are commonly used in retrospective studies. Q-Q plots were used to assess distribution of age, miles from clinic, and number of co-morbidities for patients who 
attended DGMVs and for patients who received usual care separately (See Appendices E \& F). The data do not meet the assumption of normality for those who attended DGMVs or patients who received usual care. However, the results of the parametric test are still valid due to the Central Limit Theorem.

The Chi-square test for independence indicated the patients who participated in DGMVs differed from the usual care group prior to the intervention by reporting significantly higher rates of the presence of depression, obesity and pain (See table 9). Independent t-tests showed no difference between the means for the continuous characteristics of age, miles from clinic, and number of co-morbidities between patients who attended DGMVs and patients who received usual care (See table 10). There were no other significant differences in characteristics at the beginning of the study between those who attended DGMVs and those who received usual care. 
Table 9: Differences in Characteristics of DGMVs versus Usual Care Using $\mathrm{X}^{2}$

\begin{tabular}{|c|c|c|c|c|}
\hline Characteristic & $\mathbf{N}$ & $\%$ & $\mathrm{X}^{2}$ & Sig \\
\hline \multicolumn{5}{|l|}{ Gender } \\
\hline \multicolumn{5}{|l|}{ DGMV } \\
\hline Male & 14 & 26.4 & 0.004 & 0.947 \\
\hline Female & 39 & 73.6 & & \\
\hline \multicolumn{5}{|l|}{ Usual Care } \\
\hline Male & 15 & 25.9 & & \\
\hline Female & 43 & 74.1 & & \\
\hline \multicolumn{5}{|l|}{ Marital Status } \\
\hline \multicolumn{5}{|l|}{ DGMV } \\
\hline Not Married & 29 & 54.7 & 1.083 & 0.298 \\
\hline Married & 24 & 45.3 & & \\
\hline \multicolumn{5}{|l|}{ Usual Care } \\
\hline Not Married & 26 & 44.8 & & \\
\hline Married & 32 & 55.2 & & \\
\hline \multicolumn{5}{|l|}{ Education } \\
\hline \multicolumn{5}{|l|}{ DGMV } \\
\hline Less than High School & 13 & 24.5 & 0.122 & 0.989 \\
\hline Graduated High School & 22 & 41.5 & & \\
\hline Some College & 10 & 18.9 & & \\
\hline GED & 8 & 15.1 & & \\
\hline \multicolumn{5}{|l|}{ Usual Care } \\
\hline Less than High School & 15 & 25.9 & & \\
\hline Graduated High School & 23 & 39.7 & & \\
\hline Some College & 12 & 20.7 & & \\
\hline GED & 8 & 13.8 & & \\
\hline \multicolumn{5}{|l|}{ HTN } \\
\hline \multicolumn{5}{|l|}{ DGMV } \\
\hline Has HTN & 40 & 75.5 & 0.002 & 0.962 \\
\hline No HTN & 13 & 24.5 & & \\
\hline \multicolumn{5}{|l|}{ Usual Care } \\
\hline Has HTN & 44 & 75.9 & & \\
\hline No HTN & 14 & 24.1 & & \\
\hline \multicolumn{5}{|l|}{ Kidney Disease } \\
\hline \multicolumn{5}{|l|}{ DGMV } \\
\hline Has Kidney Disease & 8 & 15.1 & 1.122 & 0.289 \\
\hline No Kidney Disease & 45 & 84.9 & & \\
\hline \multicolumn{5}{|l|}{ Usual Care } \\
\hline Has Kidney Disease & 5 & 8.6 & & \\
\hline No Kidney Disease & 53 & 91.4 & & \\
\hline \multicolumn{5}{|l|}{ Hyperlipidemia } \\
\hline \multicolumn{5}{|l|}{ DGMV } \\
\hline Has Hyperlipidemia & 28 & 52.8 & 0.968 & 0.325 \\
\hline No Hyperlipidemia & 25 & 47.2 & & \\
\hline \multicolumn{5}{|l|}{ Usual Care } \\
\hline Has Hyperlipidemia & 36 & 62.1 & & \\
\hline No Hyperlipidemia & 22 & 37.9 & & \\
\hline \multicolumn{5}{|l|}{ Heart Disease } \\
\hline \multicolumn{5}{|l|}{ DGMV } \\
\hline Has Heart Disease & 9 & 17 & 0.044 & 0.834 \\
\hline No Heart Disease & 44 & 83 & & \\
\hline Usual Care & & & & \\
\hline Has Heart Disease & 9 & 15.5 & & \\
\hline No Heart Disease & 49 & 84.5 & & \\
\hline
\end{tabular}




\begin{tabular}{|c|c|c|c|c|}
\hline \multicolumn{5}{|l|}{ Depression } \\
\hline \multicolumn{5}{|l|}{ DGMV } \\
\hline Has Depression & 24 & 45.3 & 4.583 & 0.032 \\
\hline No Depression & 29 & 54.7 & & \\
\hline \multicolumn{5}{|l|}{ Usual Care } \\
\hline Has Depression & 15 & 25.9 & & \\
\hline No Depression & 43 & 74.1 & & \\
\hline \multicolumn{5}{|l|}{ Obesity } \\
\hline \multicolumn{5}{|l|}{ DGMV } \\
\hline Has Obesity & 41 & 77.4 & 6.055 & 0.014 \\
\hline No Obesity & 12 & 22.6 & & \\
\hline \multicolumn{5}{|l|}{ Usual Care } \\
\hline Has Obesity & 32 & 55.2 & & \\
\hline No Obesity & 26 & 44.8 & & \\
\hline \multicolumn{5}{|l|}{ Pain } \\
\hline \multicolumn{5}{|l|}{ DGMV } \\
\hline Has Pain & 12 & 22.6 & 5.565 & 0.018 \\
\hline No Pain & 41 & 77.4 & & \\
\hline \multicolumn{5}{|l|}{ Usual Care } \\
\hline Has Pain & 4 & 6.9 & & \\
\hline No Pain & 54 & 93.1 & & \\
\hline \multicolumn{5}{|l|}{ Neuropathy } \\
\hline \multicolumn{5}{|l|}{ DGMV } \\
\hline Has Neuropathy & 6 & 11.3 & 1.405 & 0.236 \\
\hline No Neuropathy & 47 & 88.7 & & \\
\hline \multicolumn{5}{|l|}{ Usual Care } \\
\hline Has Neuropathy & 3 & 5.2 & & \\
\hline No Neuropathy & 55 & 94.8 & & \\
\hline \multicolumn{5}{|l|}{ Frequent Infections } \\
\hline \multicolumn{5}{|l|}{ DGMV } \\
\hline Has Frequent Infections & 3 & 5.7 & 0.315 & 0.575 \\
\hline No Frequent Infections & 50 & 94.3 & & \\
\hline \multicolumn{5}{|l|}{ Usual Care } \\
\hline Has Frequent Infections & 2 & 3.4 & & \\
\hline No Frequent Infections & 56 & 96.6 & & \\
\hline
\end{tabular}


Table 10: Differences in Characteristics of DGMVs verses Usual Care Using Independent t-tests

\begin{tabular}{lrrrr}
\hline Characteristic & Mean & SD & $\boldsymbol{t}$ & $\boldsymbol{p}$ \\
\hline Age & & & & \\
$\quad$ DGMV & 47 & 10.82 & 1.13 & 0.261 \\
$\quad$ Usual Care & 49 & 10.84 & & \\
& & & & \\
Number of Co-Morbidities & & & & \\
$\quad$ DGMV & 4.7 & 2.16 & -1.31 & 0.193 \\
$\quad$ Usual Care & 4.2 & 2.11 & & \\
& & & & \\
Distance from Clinic & & & & \\
$\quad$ DGMV & 20.7 & 19.72 & 0.25 & 0.803 \\
$\quad$ Usual Care & 21.7 & 21.08 & & \\
\hline
\end{tabular}

Aim 7: To explore the impact of dose of DGMVs on biophysical outcomes of care in uninsured persons with diabetes who receive care in a free clinic after one year.

To explore the relationship between the dosage of DGMVs and biophysical outcomes of care in uninsured persons with diabetes who receive care in a free clinic after one year, dose was collected as a continuous variable on a scale from one to six. Correlations were performed with the outcomes of body weight, BMI, $\mathrm{HgA1C}$, fasting blood glucose, serum creatinine, serum lipids, urine microalbumin, systolic blood pressure, and diastolic blood pressure at one year for those patients who attended DGMVs. Before performing a correlation analysis, scatterplots were generated to check for violation of the assumptions of linearity. The scatterplots were visually scanned for outliers. When correlations were performed, no significant correlations were found between number of DGMVs attended and biophysical outcomes of care (See table 11). However, after analyzing frequencies, it is noted that only 18 individuals attended 3 or more DGMVs. Seventeen participants attended one visit and 18 participants attended two visits. The data were then collapsed into participants who attended 3 or more group visits and those who attended less than three visits. Because $\mathrm{HgA1C}$ is so commonly used to determine an improvement in glucose levels, $\mathrm{HgA} 1 \mathrm{C}$ was also collapsed into goal met or goal not met. The American Diabetes 
Association sets the goal for $\mathrm{HgA1C}$ at less than $7 \%$. The continuous biophysical outcome of $\mathrm{HgA} 1 \mathrm{C}$ was dichotomized using $7 \%$ as a threshold to create a new variable, those who were above $7 \%$ and $7 \%$ and below. A Chi-square test was used to analyze the differences between participants who attended 3 or more group visits and those who attended less than three visits and $\mathrm{HgA1C}$ goal met or not met. There were no significant differences between the $\mathrm{HgA1C}$ met or not met outcome of patients who attended three or more DGMVs and those who attended less than three DGMVs (See Table 12).

Table 11: Correlation between Dose of DGMVs and Biophysical Outcomes

\begin{tabular}{|l|r|r|r|r|r|r|}
\hline & $\begin{array}{c}\text { Body } \\
\text { Weight } \\
\text { Time } \\
\text { Two }\end{array}$ & $\begin{array}{l}\text { BMI } \\
\text { Time } \\
\text { Two }\end{array}$ & $\begin{array}{c}\text { HgA1C } \\
\text { Time Two }\end{array}$ & $\begin{array}{c}\text { Blood } \\
\text { Glucose } \\
\text { Time } \\
\text { Two }\end{array}$ & $\begin{array}{c}\text { Creatinine } \\
\text { Time Two }\end{array}$ & $\begin{array}{c}\text { Microalbumin } \\
\text { Time Two }\end{array}$ \\
\hline Pearson & .162 & .101 & -.041 & -.014 & -.017 & -.161 \\
Correlation & & & & & \\
Sig. & .248 & .473 & .769 & .918 & .906 & .263 \\
N & 53 & 53 & 53 & 53 & 51 & 50 \\
\hline
\end{tabular}

\begin{tabular}{|c|c|c|c|c|c|c|}
\hline & $\begin{array}{c}\text { Systolic } \\
\text { Time } \\
\text { Two }\end{array}$ & $\begin{array}{c}\text { Diastolic } \\
\text { Time } \\
\text { Two }\end{array}$ & $\begin{array}{c}\text { Total } \\
\text { Cholesterol } \\
\text { Time Two }\end{array}$ & $\begin{array}{l}\text { HDL } \\
\text { Time } \\
\text { Two }\end{array}$ & $\begin{array}{l}\text { LDL Time } \\
\text { Two }\end{array}$ & $\begin{array}{c}\text { Triglycerides } \\
\text { Time Two }\end{array}$ \\
\hline Pearson & .150 & -.086 & -.156 & -.158 & .108 & -.133 \\
\hline $\begin{array}{l}\text { Correlation } \\
\text { Sig. }\end{array}$ & .284 & .540 & .269 & 263 & .459. & .357 \\
\hline $\mathbf{N}$ & 53 & 53 & 52 & 52 & 49 & 50 \\
\hline
\end{tabular}


Table 12: $\mathrm{X}^{2}$ for Dose of DGMVs \& HgA1C Goal

\begin{tabular}{ccccc}
\hline & N & \% & $\mathbf{X}^{\mathbf{2}}$ & Sig \\
\hline 2 or less DGMVs & & & & \\
HgA1C at goal & 10 & 28.6 & 0.518 & 0.323 \\
HgA1C above goal & 25 & 71.4 & & \\
3 or more DGMVs & & & & \\
HgA1C at goal & 7 & 38.9 & & \\
HgA1C above goal & 11 & 61.1 & & \\
\hline
\end{tabular}

\section{Summary}

This retrospective study was conducted with a convenience sample of patients at a free clinic in West Virginia. The majority of uninsured persons with diabetes who are cared for in this free clinic are female, white, married, with a high school education or less. There was a statistically significant decrease in systolic blood pressure from time one to time two in patients who attended DGMVs. No other significant impact on biophysical outcomes of care in persons with diabetes after attending DGMVs was noted. There was no significant impact on biophysical outcomes of care in patients who received usual care from time one to time two noted. The patients who attended DGMVs had a higher $\mathrm{HgA} 1 \mathrm{C}$ at baseline than those who received usual care. The patients who attended DGMVs continued to have a higher HgA1C after one year than those patients who received usual care. Dose of DGMVs did not impact biophysical outcomes of care in uninsured persons with diabetes who received care in a free clinic. 


\section{CHAPTER 5: SUMMARY, DISCUSSION, AND IMPLICATIONS}

The purpose of this study was to examine the relationship between attendance at Diabetes Group Medical Visits (DGMV) and biophysical outcomes of care in uninsured persons with diabetes who receive care in a free clinic. The aims of the study were to describe the characteristics of uninsured persons with diabetes cared for in a free clinic, describe the biophysical outcomes of care, explore the differences in biophysical outcomes of care before and after attending DGMVs versus receiving usual care, and explore the impact of dose of DGMVs on biophysical outcomes of care. The subjects included in this study were in poor health. DGMVs had very little impact on biophysical outcomes of care in this study. This chapter interprets the results of the data analysis guided by the major concepts of the Quality Health Outcomes Model (QHOM), presents a discussion of the findings as compared to current literature, presents the limitations of the study, and suggests implications for future practice and research.

\section{Theoretical Framework}

The theoretical framework used to guide this study was the QHOM. The four major concepts included in this model are: system, interventions, patients, and outcomes. The QHOM posits that system characteristics are the mediators of patient characteristics and interventions in producing patient outcomes (Mitchell, et al., 1998). The QHOM proposes that outcome measures should be the result of care interventions that integrate functional, social, psychological, physical, and physiologic aspects of people's experience in health and illness (Mitchell et al., 1998).The QHOM further postulates that interventions affect and are affected by both the system and patient characteristics in producing desired outcomes (Mitchell, et al., 1997). Additionally, according to the QHOM, patient characteristics can affect outcomes of care. 
The design of this study was guided by major concepts of the QHOM. The system in this study was a free clinic in North Central West Virginia, the intervention was DGMVs, the patients were low income uninsured adults, and the outcomes were biophysical measures. The environment of the clinic and the pre-existing qualifications necessary to become a patient at the free clinic affected the characteristics of the sample. Consequently, patient characteristics such as suboptimal physical condition, multiple co-morbid conditions, less education, younger age, longer driving distances to obtain care, Appalachian culture and lack of health care insurance influenced participation in the intervention and outcomes. Despite the intervention, the outcomes of care were essentially unchanged in subjects in this study, likely due to complex characteristics of persons who attended the free clinic. While the outcomes of the patients in this study were not positively impacted by the intervention, the framework of the QHOM was supported. The following paragraphs will show the relationships between patient characteristics, intervention and outcomes.

\section{Findings}

\section{Patient Characteristics}

The characteristics of uninsured adult patients who are cared for in a free clinic are not well documented in the literature. Hence, the first aim of this study was to describe the characteristics of uninsured persons with diabetes who are cared for in a free clinic. This study described age, gender, ethnicity, marital status, duration of DM, education, miles from clinic, comorbidities, and depression in this population. Participants had a mean of 5 co-morbid conditions other than diabetes. The majority of the patients were female, white, had a high-school education or less, and were age 50 or younger. The subjects being cared for in this free clinic drove long distances to receive care. 
Number of co-morbid conditions has been documented to affect outcomes of care. Patients with a greater overall number of co-morbidities place lower priority on diabetes and have worse diabetes self-management ability scores (Glasgow, et al., 1996). Self-management of diabetes, such as checking glucose levels, taking medications, and adhering to dietary and activity recommendations is necessary to maintain optimal biophysical outcomes of care (American Diabetes Association, 2010).

Gender has been documented to affect outcomes such as rates of obesity, amount of physical activity, and adherence. Females have a greater prevalence of obesity compared to males (Ferraro et al., 1992). Obesity causes insulin resistance which contributes to decreased effectiveness in lowering blood glucose. The resulting increase in blood glucose may raise levels outside the normal range and cause adverse health effects (McPhee, 2011). Men with diabetes have been found to be more physically active than women with diabetes (Carpenter, 1998) which may significantly lower cardiovascular risks and overall mortality (Church et al., 2004). Further, women have been found to consume more calories, make poor food choices and have lower levels of adherence than men (Whitlock, et al., 1997).

Nearly all participants in this study were white. In order to receive care at this free clinic, subjects must have resided in West Virginia. West Virginia is in the only state that is entirely in Appalachia (AppalachianRegionalCommission, Retrieved 2011-06-04). Although this study did not collect data on culture, all of the participants of this study live in West Virginia. According to the 2000 United States Census, 74.2 percent of people residing in West Virginia are native to West Virginia (U.S.CensusBureau, 2000). Hence, it is the assumption of the researcher that the subjects of this study are members of Appalachian culture, and therefore subscribe to some of the social norms of the culture. These norms and beliefs affect the patients' desire to interact with a 
care delivery system (Holzemer, 1994). People from Appalachian culture possess core values such as individualism, self-reliance, and fatalism (Smith \& Tessaro, 2005). These core values may affect a patient's willingness to share personal information with outsiders. An outsider can be any person that is not familiar to the patient such as other patients and health care providers participating in group visits. In order for DGMVs to affect outcomes of care, each patient must share similar experiences and be willing to participate in a group (Barud, et al., 2006).

Education level also appears to have an effect on participation in medical decision making and thus may impact outcomes of care (DeWalt, et al., 2007). Over one third of subjects in this study did not graduate high school. Educational level has been shown to be significant in disease control of persons with diabetes. On the National Health and Nutrition Examination Survey (NHANES) from 1999 to 2006, those persons with diabetes who had a high school education or greater had consistently shown improved outcomes such as decreased HgA1C, decreased blood pressure and decreased total cholesterol levels than those who were less educated (McWilliams, et al., 2009).

The age of all subjects in this study is less than 65 and the majority of the subjects were age 50 and younger. Younger adult patients, less than 60 years old, are significantly less likely to attend education programs and multiple healthcare visits than older adult patients (Abdulwadud et al., 1997). Additionally, the largest reductions in $\mathrm{HgAlC}$ have been documented in patients who attend more healthcare visits (Brown et al., 2005). Consequently, lack of attendance to multiple healthcare visits and education programs by younger populations may contribute to decreased effectiveness of interventions.

Longer driving distances from home to the site of primary care have been associated with poorer outcomes in rural subjects (Strauss, et al., 2006). The majority of subjects in this study 
live greater than twenty miles from the clinic making both traveling time to clinic and transportation difficult. Living far away from primary health care centers, particularly in West Virginia presents multiple barriers to care. These barriers include inability to quickly access care due to distance, lack of an interstate transportation system, lack of public transportation systems, and cost of transportation (Arcury, Preisser, Gesler, \& Powers, 2005). These barriers affect a person's ability and willingness to obtain needed care (Arcury et al., 2005).

\section{Biophysical Outcomes Prior to Intervention}

The biophysical outcomes of patients who are cared for in a free clinic are not well documented in the literature. The second aim of this study was to describe the biophysical outcomes of care in uninsured persons with diabetes who are cared for in a free clinic. The outcomes examined in this study were body weight, BMI, fasting blood glucose, $\mathrm{HgA1C}$, creatinine, lipids, blood pressure, and microalbumin. An outcome is said to be a component of a patient's clinical and functional status after an intervention has been applied (Barr, et al., 2001). The biophysical outcomes of care of uninsured persons with diabetes who are cared for in this free clinic indicate suboptimal control of multiple co-morbid conditions.

The mean BMI was in the severe obesity category for this sample of patients (Sturm, 2007). Only $5 \%$ of this sample of patients had a BMI indicating normal body weight. The remaining $95 \%$ of this sample of patients were in the overweight to morbid obesity categories. According to the Standards of Medical Care released by the American Diabetes Association, weight loss has been shown to reduce insulin resistance. Insulin resistance leads to higher levels of blood glucose. The resulting increase in blood glucose can cause adverse health effects (McPhee, 2011). 
The mean $\mathrm{HgA} 1 \mathrm{C}$ in this population was 8.09 percent. Having a $\mathrm{HgAlC}$ above 8 percent means that the average daily blood glucose of this sample of persons with diabetes is above 200mg/dl indicating significant chronic hyperglycemia (AmericanDiabetesAssociation, 2010). Current recommendations are set at achieving and maintaining a $\mathrm{HgAlC}$ of less than $7 \%$ for most patients (Nathan et al., 2009). More than $60 \%$ of the patients in this sample have $\mathrm{HgA1C}$ levels higher than recommended treatment goals. The American Diabetes Association suggests lowering $\mathrm{HgA} 1 \mathrm{C}$ to below or around $7 \%$ in order to reduce microvascular and neuropathic complications of diabetes which contribute to blindness, chronic kidney disease, and lower limb amputations.

While creatinine levels were normal in this sample, urine microalbumin was elevated. Maintaining normal creatinine levels and urine microalbumin reduce the risk of macrovascular disease. Persistent elevated urine microalbumin has been shown to be the earliest indication of diabetic nephropathy in diabetes patients (Garg JP, 2002). The level of microalbumin in this sample was in the range that indicates diabetic nephropathy. Microalbuminuria and nephropathy are also a well-established markers of increased coronary vascular disease risk (Garg JP, 2002).

The lipid levels of this sample indicated dyslipidemia. According to the American Diabetes Association, patients with diabetes have an increased prevalence of lipid abnormalities, which contributes to their high risk of coronary vascular disease. According to the American Diabetes Association, low levels of HDL cholesterol associated with elevated LDL and triglyceride levels, which are seen in this population, are the most prevalent pattern of dyslipidemia in persons with diabetes. Elevating HDL and lowering LDL and triglyceride levels are crucial to preventing stroke, myocardial infarction, and other vascular complications (AmericanDiabetesAssociation, 2010). 


\section{Usual Care Group Outcomes}

The forth aim of this study was to compare biophysical outcomes of care in patients who received usual care at baseline and again after one year. There were no significant differences between biophysical outcomes of care from time one to time two noted in those who received usual care. The subjects in this group were severely obese, with elevated HgA1C levels, nephropathy, and dyslipidemia at baseline and continued to be in sub-optimal physical condition after one year of usual care.

The majority of patients who received usual care in this free clinic had five or more visits to the clinic in the course of one year. Persons with diabetes who are treated with insulin should be seen by their healthcare provider at least every three to four months. Those who are treated with oral medications or who are managing diabetes through diet should be seen at least every four to six months (AmericanDiabetesAssociation, 2010). Hence, the subjects in this study who received usual care attended the clinic and received care at least as often as recommended by the ADA, if not more frequently. However, attending the clinic and receiving usual care did not affect outcomes of care in this sample of patients. These findings are similar to previous findings related to usual diabetes care. Despite advancing treatment options and providing ongoing diabetes care, biophysical outcomes of diabetes care continue to be less than optimal (SERVICES, et al., 2008).

\section{DGMV Intervention Group Outcomes}

There were differences in the biophysical outcomes found in the sample of patients who attended DGMVs. Previous to the intervention, $\mathrm{HgA1C}$ was elevated in the patients who attended DGMVs and remained elevated after one year. Maintaining high $\mathrm{HgA1C}$ levels increases the risk of long-term complications of diabetes. Mean systolic blood pressure 
decreased to acceptable levels based on clinical guidelines (AmericanDiabetesAssociation, 2002) in the patients who attended DGMVs after one year. People with both diabetes and hypertension have approximately twice the risk of cardiovascular disease than patients who have hypertension alone. Hence, reducing and maintaining blood pressure can decrease cardiovascular risk. However, there were no other significant differences in biophysical outcomes of care in the patients who participated in DGMVs after one year of care.

The biophysical outcomes reported in other literature related to DGMVs showed that participants started nearer to treatment goals prior to intervention than the sample of patients in this study (Chiu, et al., 2009; Clancy, Brown, et al., 2003; Clancy, Yeager, et al., 2007; CulhanePera, et al., 2005; Guzek, et al., 2009; Trento, et al., 2002; Trento, et al., 2001; Wagner, et al., 2001). Most studies reviewed related to DGMVs reported HgA1C levels from 6.9-7.6 \% (Keyserling et al., 2002; Wagner, 1998; Wagner, et al., 2001). Nearly 70\% of the sample of patients who attended DGMVs had HgA1C levels above treatment goals at time one. Additionally, greater than $62 \%$ of the sample of patients who attended DGMVs had $\mathrm{HgA1C}$ levels above what has been previously seen in the literature. However, while $\mathrm{HgAlc}$ values are reported in most of the DGMV literature, other biophysical outcomes of care are inconsistently studied, making comparisons difficult. Suboptimal biophysical outcomes and complex patient characteristics of this sample make implementing interventions complex and perhaps less effective than in other populations.

Another aim of this study was to explore the impact of dose of DGMVs on biophysical outcomes of care. Participants were able to attend up to six DGMVs in which they would be provided education about blood glucose monitoring, medication, nutrition, exercise, foot care, heart disease, complications including sick day care, and behavior changes. In addition to their 
regular clinic visits, the patients can attend the clinic monthly until they have received all of the education offered by DGMVs. There was no significant relationship found between number of DGMVs attended and biophysical outcomes of care in this study. However, it is important to note that the majority of patients attended two or less DGMVs in one year. Previous studies reviewed related to DGMVs suggest that improved interventions are seen in those patients who attend DGMVs more frequently (Beck, et al., 1997; Trento, et al., 2002; Trento, et al., 2001). Other studies that reported improvement in measured biophysical outcomes related to participation in DGMVs measured outcomes after at least 2 years of care (Clancy, Huang, et al., 2007; Trento, et al., 2002). Hence, the lack of improvement in biophysical outcomes of care in this sample of patients who attended DGMVs may be due to low attendance rates or less time between intervention and outcome measurement than in previous studies.

\section{Comparison of Usual Care and DGMV Intervention group outcomes}

There were differences in patient characteristics in the sample of patients who attended DGMVs versus those who received usual care. Similar to the usual care group, the DGMV group had elevated $\mathrm{HgA1C}$ levels, nephropathy, and dyslipidemia. However, patients who participated in DGMVs had higher depression scores, were more obese and reported to have pain more frequently than patients who received usual care in this study. Patients with depression are more likely to experience complications of diabetes, have worse glycemic control, and be less adherent to self-care behaviors than patients who are not depressed (J. O. Prochaska, 2008). Pain has been found to limit a person's ability to perform self-management behaviors (Krein, Heisler, Piette, Makki, \& Kerr, 2005). Obesity increases the incidence of insulin resistance, hypertension, dyslipidemia, and cardiovascular disease (DeFronzo \& Ferrannini, 1991). These group 
differences could have contributed to diminished response to the intervention when compared to the usual care group.

While this study did not measure improvements to the process of providing healthcare, an unexpected healthcare system improvement was found. Traditionally, DGMVs have been delivered by physicians with the assistance of nurses or diabetes educators in fee for service healthcare organizations. The intervention studied here employed a Nurse Practitioner and a PharmD who is a Diabetes Educator. This innovative collaborative approach to deliver care resulted in urine microalbumin being measured and charted more frequently in the subjects who attended DGMVs. Having the entire picture of the patients health status by reviewing previously charted biophysical outcomes of care allows the healthcare team to make more informed decisions regarding the future care of the patient (Honoré, 2010). Thus, this finding suggests that a collaborative approach may improve the process of providing care even if biophysical outcomes of care remained essentially unchanged for both participants of DGMVs and usual care patients.

\section{Implications for Practice}

Clinical outcomes associated with group visits have been documented to include decreased or stable $\mathrm{HgA1C}$, decreased cardiovascular risk, decreased or stable BMI, decreased LDL, increased HDL, decreased or stable blood pressure and slowed progression of retinopathy (Clancy, Cope, et al., 2003). However, DGMVs were not effective in improving biophysical outcomes of care in the population of persons with diabetes cared for in this free clinic. In the future, assessment of humanistic outcomes such as quality of life improvement, improved patient care delivery, and improved patient satisfaction may be useful in assessing the effectiveness of DGMVs in this population. The implementation of DGMVs may be a viable option for 
improving biophysical outcomes of care in some patient populations. Prior to implementation of DGMVs as an intervention, assessment of both the characteristics of the patients to be cared for and the system in which DGMVs will take place is advised.

The patient characteristics found in previous studies to contribute to the success of DGMVs as an intervention include ethnic minority groups, female gender, older age, and some type of health care insurance. The results of the current study suggest that other patient characteristics such as pre-existing multiple co-morbid conditions other than diabetes, education level, depression, pain, and distance to the clinic can negatively affect biophysical outcomes of care and the impact of DGMVs. Future interventions for this population should be tailored to treat people who have diabetes and multiple co-morbid conditions, depression, pain, and live long distances from the clinic The addition of services from other disciplines such as social work or behavioral health for this population may contribute to improved outcomes.

In addition to tailoring interventions based on patient characteristics, an assessment of the healthcare delivery system is necessary. This study tested an intervention that was originally designed to operate within a traditional healthcare delivery system. The system of interest, the free clinic, cannot operate in the same ways as fee for service practices. Future interventions that investigate changes in the healthcare delivery system are warranted. In addition to the Nurse Practitioner and Pharm D, other healthcare professionals are needed to address the severe obesity, poor physical condition, and macrovascular complications seen in this population. Instead of an additional health care visit that is meant to supplement individual healthcare, clustered care visits where a multidisciplinary health care team work together to assess, diagnose, treat and educate are needed (Funnell, 2004). Another idea might be the use of innovative technologies or the use of home care services to provide distance care and 
individualized education for this population. Based on these study results, implementation of DGMVs is only suggested if the clinic is easily accessible and can provide care from multiple healthcare team members. New interventions will require not only a change in practice for primary health care providers but in the healthcare delivery system.

\section{Future Research}

Future research with this population should focus on the unique needs of persons with diabetes who receive care in free clinics. It is clear that this population is different than those previously studied using DGMVs as an intervention. In 2008, the National Center for Health Statistics reported that 46 million individuals under the age of 65 were uninsured, which translates to 16.8 percent of the population of adults under the age of 65 without insurance (CDC, 2006). Differing characteristics of uninsured patients such as obesity, multiple co-morbid conditions, less education, younger age, longer driving distances, Appalachian culture, and low incomes provide target areas for future tailored intervention research.

Future research should include multi-site randomized clinical trials with consistent measures of biophysical outcomes related to DGMVs. Randomization to treatment group would correct self-selection to the intervention and non-equal groups as seen in this study. Additionally, future research should control for the dose of the intervention and separate the researcher role from clinician role. While $\mathrm{HgA} 1 \mathrm{c}$ values are reported outcome measures in most of the DGMV literature, other biophysical outcomes of care are inconsistently reported. Prospective studies are needed to evaluate the biophysical outcomes of care in persons with diabetes when innovative care models are used. Such biophysical outcomes include body weight, BMI, $\mathrm{HgA1C}$, fasting blood glucose, serum creatinine, serum lipids, urine microalbumin, and blood pressure. Measuring and reporting consistent biophysical outcomes as suggested by the American 
Diabetes Association will assist researchers and clinicians in comparing the impact of DGMVs on outcomes.

Many factors have been implicated in affecting outcomes for persons with diabetes. This study only investigated biophysical outcomes of care. This study did not investigate other outcome measures such as improving quality of life or improvements in the process of providing care. Previous research has documented that those persons with diabetes who were willing participate in DGMVs experienced an increase in satisfaction with care, interaction with providers, diabetes knowledge, education, quality of life and preventive procedures and screenings (Trento, et al., 2001; Wagner, et al., 2001). Additionally, positive outcomes have been achieved in other populations with an increase in provider trust and a decrease or more effective use of provider time (Clancy, Cope, et al., 2003). Longitudinal studies are needed to investigate how improved quality of life, provider relationships, and knowledge of disease processes could impact long-term negative consequences of diabetes.

\section{Limitations}

The study design was based on a convince sample of persons with diabetes who attended at a free clinic in West Virginia from May 2007 to August 18, 2009. The generalizability of results is limited to the specific population of the study, given that the sample consisted of predominantly white, middle-aged females. Furthermore, due to the retrospective nature of the study, only the variables present in the chart could be collected.

This study did not take in to consideration the barriers to attending DGMVs for this population. Out of the possible 326 patients who received care at the clinic during the study timeframe, only 111 patients could be included in the study. The participants who were excluded did not have two visits within one year during the study timeframe and hence, one year 
comparisons could not be made. Attendance at clinic appointments was unpredictable, with the cancellation rate being high for many patients. Many factors have been implicated in affecting outcomes, such as readiness for change, lack of transportation, financial burden, culture, age, gender, co-morbid conditions, and knowledge. Future prospective studies could personalize interventions towards individual patients and their families, cluster care, assess for readiness to change, and address financial burden. Such studies would address the barriers to attending DGMVs found in this population.

Another limitation is the ability of the study to examine confounding factors that may influence patient characteristics and biophysical outcomes of care. Out of the possible 326 patients who received care at the clinic during the study timeframe, only 111 patients could be included in the study. The participants who were excluded did not have two visits within one year during the study timeframe and hence, one year comparisons could not be made. Attendance at clinic appointments is unpredictable, with the cancellation rate being high for many patients. Many factors have been implicated in affecting outcomes, such as low attendance rates, lack of social support, financial burden, decreased access to care, culture, and knowledge. This study only collected demographic and outcome variables available in the chart.

One more limitation of the study is missing data. The patients who received usual care had depression scores missing from 20 cases (18\%). Depression score was analyzed as a characteristic to describe the groups prior to intervention, not to compare the effectiveness of the intervention. However, because patients with depression are more likely to experience complications of diabetes, have worse glycemic control, and be less adherent to self-care behaviors than patients who are not depressed, examining differences in depression data before and after intervention could be meaningful. Microalbumin had 27\% (N=30) missing data at 
time one and $29.7 \%(\mathrm{~N}=33)$ missing data at time two from the entire sample. Patients who attended DGMVs had no missing data in microalbumin at time one and 3 cases $(2.7 \%)$ were missing microalbumin data at time two. Patients who received usual care were missing microalbumin in 30 cases (27\%) at time one and 30 cases (27\%) at time two. The observed power for microalbumin data may not be enough detect a significant difference in microalbumin in those who attended DGMVs versus usual care at time one and time two. The large amount of missing microalbumin data for the patients who received usual care is a limitation of the study. It is also noted that there is a large standard deviation in urine microalbumin. The microalbumin values are known to be actual patient results and not aberrant data entry mistakes. Hence, the values were included in the data evaluation.

\section{Conclusions}

The persons with diabetes who were cared for in this clinic were severely obese, with elevated $\mathrm{HgA} 1 \mathrm{C}$ levels, nephropathy, and dyslipidemia. In addition to suboptimal physical condition, the characteristics of persons with diabetes who receive care at this free clinic such as multiple co-morbid conditions, less education, younger age, longer driving distances to obtain care, Appalachian culture, and lack of health care insurance may have contributed to the lack of improvement in biophysical outcomes of care in this population. DGMVs have been shown in the literature to improve biophysical outcomes. However, DGMV as an intervention is not enough to improve biophysical outcomes in this population. Interventions targeted to the unique characteristics of this population are needed to prevent devastating complications. Such interventions should not only cluster care, but also include improved access to care and access to an interprofessional team. The addition of services from other disciplines such as social work or behavioral health and the use of innovative technologies or home care services for this 
population may contribute to improved outcomes. Longitudinal studies are needed to investigate not only biophysical outcomes of care but how improved quality of life, provider relationships, and knowledge of disease processes could impact long-term negative consequences of diabetes. 


\section{REFERENCES}

Abdulwadud, O., Abramson, M., Forbes, A., James, A., Light, L., Thien, F. (1997). Attendance at an asthma educational intervention: characteristics of participants and non-participants. Respir Med, 91(9), 524-529. doi: S0954-6111(97)90085-8 [pii]

American Diabetes Association. (2010). Standards of Medical Care in Diabetesâ€ 2011.

Diabetes Care, 34(Supplement 1), S11-S61. doi: 10.2337/dc11-S011

AmericanDiabetesAssociation. (2002). Treatment of Hypertension in Adults With Diabetes.

Diabetes Care, 25(suppl 1), s71-s73. doi: 10.2337/diacare.25.2007.S71

AmericanDiabetesAssociation. (2010). Executive Summary: Standards of Medical Care in

Diabetes. Diabetes Care, 33(Supplement 1), S4-S10. doi: 10.2337/dc10-S004

Andres, R. (1971). Aging and Diabetes. Medical Clinics of North America, 55, 835-846.

AppalachianRegionalCommission. (Retrieved 2011-06-04). Counties in Appalacia. http://www.arc.gov/index.do?nodeId=2

Arcury, T. A., Gesler, W. M., Preisser, J. S., Sherman, J., Spencer, J., \& Perin, J. (2005). The effects of geography and spatial behavior on health care utilization among the residents of a rural region. Health Serv Res, 40(1), 135-155. doi: HESR346 [pii]

10.1111/j.1475-6773.2005.00346.x

Arcury, T. A., Preisser, J. S., Gesler, W. M., \& Powers, J. M. (2005). Access to transportation and health care utilization in a rural region. J Rural Health, 21(1), 31-38.

Ayanian, J. Z., Weissman, J. S., Schneider, E. C., Ginsburg, J. A., \& Zaslavsky, A. M. (2000). Unmet health needs of uninsured adults in the United States. JAMA, 284(16), 2061-2069. doi: joc00915 [pii] 
Barr, J. T., Schumacher, G., \& Myers, E. F. (2001). Case Problem: Quality of Life Outcomes Assessment: How Can You Use it in Medical Nutrition Therapy? Journal of the American Dietetic Association, 101(9), 1064-1066.

Barud, S., Marcy, T., Armor, B., Chonlahan, J., \& Beach, P. (2006). Development and implementation of group medical visits at a family medicine center. Am J Health Syst Pharm, 63(15), 1448-1452. doi: 63/15/1448 [pii]

10.2146/ajhp050266

Beck, A., Scott, J., Williams, P., Robertson, B., Jackson, D., Gade, G. (1997). A randomized trial of group outpatient visits for chronically ill older HMO members: the Cooperative Health Care Clinic. J Am Geriatr Soc, 45(5), 543-549.

Bray, P., Roupe, M., Young, S., Harrell, J., Cummings, D. M., \& Whetstone, L. M. (2005). Feasibility and effectiveness of system redesign for diabetes care management in rural areas: the eastern North Carolina experience. Diabetes Educ, 31(5), 712-718. doi: $31 / 5 / 712$ [pii]

$10.1177 / 0145721705280830$

Bray, P., Thompson, D., Wynn, J. D., Cummings, D. M., \& Whetstone, L. (2005). Confronting disparities in diabetes care: the clinical effectiveness of redesigning care management for minority patients in rural primary care practices. J Rural Health, 21(4), 317-321.

Brown, S. A., Blozis, S. A., Kouzekanani, K., Garcia, A. A., Winchell, M., \& Hanis, C. L. (2005). Dosage Effects of Diabetes Self-Management Education for Mexican Americans. Diabetes Care, 28(3), 527-532. doi: 10.2337/diacare.28.3.527 
Carpenter, W., Fonong T, Toth MJ, Ades PA, Calles-Escandon J, Walston JD, Poehlman ET (1998). Total daily energy expenditure in free-living older African-Americans and Caucasians. Am J Physiol, 274, E96-E101.

CDC. (2006). National Center for Health Statistics. Hyattsville, MD 20782.

Chiu, Y. W., Chang, J. M., Lin, L. I., Chang, P. Y., Lo, W. C., Wu, L. C. (2009). Adherence to a diabetic care plan provides better glycemic control in ambulatory patients with diabetes. Kaohsiung J Med Sci, 25(4), 184-192.

Church, T. S., Cheng, Y. J., Earnest, C. P., Barlow, C. E., Gibbons, L. W., Priest, E. L. (2004). Exercise capacity and body composition as predictors of mortality among men with diabetes. Diabetes Care, 27(1), 83-88.

Clancy, D. E., Brown, S. B., Magruder, K. M., \& Huang, P. (2003). Group visits in medically and economically disadvantaged patients with diabetes and their relationships to clinical outcomes. Top Health Inf Manage, 24(1), 8-14.

Clancy, D. E., Cope, D. W., Magruder, K. M., Huang, P., Salter, K. H., \& Fields, A. W. (2003). Evaluating group visits in an uninsured or inadequately insured patient population with uncontrolled diabetes. Diabetes Educ, 29(2), 292-302.

Clancy, D. E., Dismuke, C. E., Magruder, K. M., Simpson, K. N., \& Bradford, D. (2008). Do diabetes group visits lead to lower medical care charges? Am J Manag Care, 14(1), 3944. doi: 6968 [pii]

Clancy, D. E., Huang, P., Okonofua, E., Yeager, D., \& Magruder, K. M. (2007). Group visits: promoting adherence to diabetes guidelines. J Gen Intern Med, 22(5), 620-624. doi: $10.1007 / \mathrm{s} 11606-007-0150-3$ 
Clancy, D. E., Yeager, D. E., Huang, P., \& Magruder, K. M. (2007). Further evaluating the acceptability of group visits in an uninsured or inadequately insured patient population with uncontrolled diabetes. Diabetes Educ, 33(2), 309-314. doi: 33/2/309 [pii]

$10.1177 / 0145721707299266$

Culhane-Pera, K., Peterson, K. A., Crain, A. L., Center, B. A., Lee, M., Her, B. (2005). Group visits for Hmong adults with diabetes mellitus: a pre-post analysis. $J$ Health Care Poor Underserved, 16(2), 315-327. doi: S1548686905203157 [pii]

10.1353/hpu.2005.0030

Darnell, J. S. (2010). Free Clinics in the United States. Archives of Internal Medicine, 170(11), 946-953.

Deeb-Sossa, N., Perreira, K. , Harris, K. and Bollen, K. A. . (2003). "What are we Measuring? An Evaluation of The CES-D Across Race/Ethnicity and Immigrant Generation". Paper presented at the annual meeting of the American Sociological Association, 2009

DeFronzo, R. A., \& Ferrannini, E. (1991). Insulin resistance. A multifaceted syndrome responsible for NIDDM, obesity, hypertension, dyslipidemia, and atherosclerotic cardiovascular disease. Diabetes Care, 14(3), 173-194.

Dennis, C. L. (2003). Peer support within a health care context: a concept analysis. Int J Nurs Stud, 40(3), 321-332. doi: S0020748902000925 [pii]

DeWalt, D. A., Boone, R. S., \& Pignone, M. P. (2007). Literacy and its relationship with selfefficacy, trust, and participation in medical decision making. Am J Health Behav, 31 Suppl 1, S27-35. doi: 10.5555/ajhb.2007.31.supp.S27 
Ferraro, R., Lillioja, S., Fontvieille, A. M., Rising, R., Bogardus, C., \& Ravussin, E. (1992). Lower sedentary metabolic rate in women compared with men. J Clin Invest, 90(3), 780784. doi: 10.1172/JCI115951

Funnell, M. M. (2004). Patient empowerment. Crit Care Nurs Q, 27(2), 201-204.

Garg JP, B. G. ( 2002). Microalbuminuria: marker of vascular dysfunction, risk factor for cardiovascular disease. . Vasc Med(7), 35-43.

Glasgow, R. E., Davidson, K. W., Dobkin, P. L., Ockene, J., \& Spring, B. (2006). Practical behavioral trials to advance evidence-based behavioral medicine. Ann Behav Med, 31(1), 5-13. doi: 10.1207/s15324796abm3101_3

Glasgow, R. E., Toobert, D. J., \& Hampson, S. E. (1996). Effects of a brief office-based intervention to facilitate diabetes dietary self-management. Diabetes Care, 19(8), 835842.

Guzek, J., Guzek, S., Murphy, K., Gallacher, P., \& Lesneski, C. (2009). Improving Diabetes Care Using a Multitiered Quality Improvement Model. Am J Med Qual. doi: 1062860609346348 [pii]

$10.1177 / 1062860609346348$

Holzemer, W. L. (1994). The impact of nursing care in Latin America and the Caribbean: a focus on outcomes. J Adv Nurs, 20(1), 5-12.

Honoré, P. A., \& Scott, W. . (2010). Priority areas for improvement of quality in public health. Washington, DC.

http://www.encyclopedia.com/doc/1G2-3045300301.html, E. c. (Producer). (2008, Retrieved April 05, 2011). "Central Limit Theorem.". 
Jaber, R., Braksmajer, A., \& Trilling, J. (2006). Group Visits for Chronic Illness Care: Models, Benefits and Challenges. Family Practice Management, 13(1), 37-40.

Kerr, E. A., Heisler, M., Krein, S. L., Kabeto, M., Langa, K. M., Weir, D. (2007). Beyond comorbidity counts: how do comorbidity type and severity influence diabetes patients' treatment priorities and self-management? J Gen Intern Med, 22(12), 1635-1640. doi: $10.1007 / \mathrm{s} 11606-007-0313-2$

Keyserling, T. C., Ammerman, A. S., Samuel-Hodge, C. D., Ingram, A. F., Skelly, A. H., Elasy, T. A. (2000). A diabetes management program for African American women with diabetes. Diabetes Educ, 26(5), 796-805.

Keyserling, T. C., Samuel-Hodge, C. D., Ammerman, A. S., Ainsworth, B. E., HenriquezRoldan, C. F., Elasy, T. A. (2002). A randomized trial of an intervention to improve selfcare behaviors of African-American women with diabetes: impact on physical activity. Diabetes Care, 25(9), 1576-1583.

Krein, S. L., Heisler, M., Piette, J. D., Makki, F., \& Kerr, E. A. (2005). The effect of chronic pain on diabetes patients' self-management. Diabetes Care, 28(1), 65-70. doi: 28/1/65 [pii]

Leventhal H, L. E., Cameron L. . (2001). Representations, procedures, and affect in illness selfregulation: A perceptual-cognitive model. In S. J. Baum A (Ed.), Handbook of health psychology. (pp. 19 - 47). Mahwah, NJ: Erlbaum.

Maddigan, S. L., Majumdar, S. R., \& Johnson, J. A. (2005). Understanding the complex associations between patient-provider relationships, self-care behaviours, and healthrelated quality of life in diabetes: a structural equation modeling approach. Qual Life Res, 14(6), 1489-1500. 
Mazze RS, D. D., Strock E, Peterson K, McClave CR, Meszaros JF, Leigh C, Owens LW, Deeb LC, Peterson A, Kummer M. (1994). Staged diabetes management toward an integrated model of diabetes care. . Diabetes Care 17 (Suppl. 1), 56-66.

Mc Manus, V., \& Savage, E. Cultural perspectives of interventions for managing diabetes and asthma in children and adolescents from ethnic minority groups. Child: Care, Health and Development, 36(5), 612-622. doi: 10.1111/j.1365-2214.2010.01101.x

McPhee, S. P., M. (Ed.). (2011). Current medical diagnosis and treatment 2011 (50th ed ed.). NY: Lange Medical Books/McGraw-Hill.

McWilliams, J. M., Meara, E., Zaslavsky, A. M., \& Ayanian, J. Z. (2009). Differences in control of cardiovascular disease and diabetes by race, ethnicity, and education: U.S. trends from 1999 to 2006 and effects of medicare coverage. Ann Intern Med, 150(8), 505-515. doi: 150/8/505 [pii]

Mitchell, P. H., Ferketich, S., \& Jennings, B. M. (1998). Quality health outcomes model. American Academy of Nursing Expert Panel on Quality Health Care. Image J Nurs Sch, $30(1), 43-46$.

Mitchell, P. H., Heinrich, J., Moritz, P., \& Hinshaw, A. S. (1997). Outcome measures and care delivery systems. Introduction and purposes of conference. Med Care, 35(11 Suppl), NS1-5.

Nathan, D. M., Buse, J. B., Davidson, M. B., Ferrannini, E., Holman, R. R., Sherwin, R. (2009). Medical management of hyperglycemia in diabetes: a consensus algorithm for the initiation and adjustment of therapy: a consensus statement of the American Diabetes Association and the European Association for the Study of Diabetes. Diabetes Care, 32(1), 193-203. doi: dc08-9025 [pii] 
$10.2337 / \mathrm{dc} 08-9025$

Philis-Tsimikas, A., Walker, C., Rivard, L., Talavera, G., Reimann, J. O., Salmon, M. (2004). Improvement in diabetes care of underinsured patients enrolled in project dulce: a community-based, culturally appropriate, nurse case management and peer education diabetes care model. Diabetes Care, 27(1), 110-115.

Prevention, C. f. D. C. a. (2007). National diabetes fact sheet: general information and national estimates on diabetes in the United States. Atlanta, GA.

Prochaska, J. J., Nigg, C. R., Spring, B., Velicer, W. F., \& Prochaska, J. O. The benefits and challenges of multiple health behavior change in research and in practice. Prev Med, 50(1-2), 26-29. doi: S0091-7435(09)00595-7 [pii]

10.1016/j.ypmed.2009.11.009

Prochaska, J. O. (2008). Decision making in the transtheoretical model of behavior change. Med Decis Making, 28(6), 845-849. doi: 0272989X08327068 [pii]

10.1177/0272989X08327068

Promotion., N. C. f. C. D. P. a. H. (Accessed September 20, 2010). Diabetes Public Health Resource, from Available at: http://www.cdc.gov/diabetes/pubs/estimates.htm.

Rosswurm, M. A., \& Larrabee, J. H. (1999). A model for change to evidence-based practice. Image J Nurs Sch, 31(4), 317-322.

Ryan, J. G. (2009). Cost and policy implications from the increasing prevalence of obesity and diabetes mellitus. Gend Med, 6 Suppl 1, 86-108. doi: S1550-8579(09)00003-5 [pii]

10.1016/j.genm.2009.01.002 
Sabate, E., World Health, O., Project, W. H. O. A. t. L. T. T., \& Global Adherence Interdisciplinary, N. (2003). Adherence to long-term therapies : evidence for action. Geneva :: World Health Organization.

Schillinger, D., Hammer, H., Wang, F., Palacios, J., McLean, I., Tang, A. (2007). Seeing in 3-D: Examining the Reach of Diabetes Self-Management Support Strategies in a Public Health Care System. Health Educ Behav. doi: 1090198106296772 [pii]

$10.1177 / 1090198106296772$

SERVICES, U. S. D. O. H. A. H., Prevention, C. f. D. C. a., \& Statistics, N. C. f. H. (2008). Summary Health Statistics for U.S. Adults: National Health Interview Survey, 2008. Hyattsville, Maryland.

Smith, S. L., \& Tessaro, I. A. (2005). Cultural perspectives on diabetes in an Appalachian population. Am J Health Behav, 29(4), 291-301.

Strauss, K., MacLean, C., Troy, A., \& Littenberg, B. (2006). Driving distance as a barrier to glycemic control in diabetes. J Gen Intern Med, 21(4), 378-380. doi: JGI386 [pii]

10.1111/j.1525-1497.2006.00386.x

Sturm, R. (2007). Increases in morbid obesity in the USA: 2000-2005. Public Health, 121(7), 492-496. doi: S0033-3506(07)00012-1 [pii]

10.1016/j.puhe.2007.01.006

Trento, M., Passera, P., Bajardi, M., Tomalino, M., Grassi, G., Borgo, E. (2002). Lifestyle intervention by group care prevents deterioration of Type II diabetes: a 4-year randomized controlled clinical trial. Diabetologia, 45(9), 1231-1239. doi: $10.1007 / \mathrm{s} 00125-002-0904-8$ 
Trento, M., Passera, P., Tomalino, M., Bajardi, M., Pomero, F., Allione, A. (2001). Group visits improve metabolic control in diabetes: a 2-year follow-up. Diabetes Care, 24(6), 9951000.

U.S.CensusBureau. (2000). DP-2 Profile of West Virginia Social Characteristics. Retrieved from http://factfinder.census.gov/servlet/QTTable?_bm=n\&_lang=en\&qr_name=DEC_2000_S F3_U_DP2\&ds_name=DEC_2000_SF3_U\&geo_id=04000US54.

van Dam, H. A., van der Horst, F. G., Knoops, L., Ryckman, R. M., Crebolder, H. F., \& van den Borne, B. H. (2005). Social support in diabetes: a systematic review of controlled intervention studies. Patient Educ Couns, 59(1), 1-12. doi: S0738-3991(04)00365-9 [pii]

10.1016/j.pec.2004.11.001

Wagner, E. H. (1998). Chronic disease management: what will it take to improve care for chronic illness? Eff Clin Pract, 1(1), 2-4.

Wagner, E. H., Grothaus, L. C., Sandhu, N., Galvin, M. S., McGregor, M., Artz, K. (2001). Chronic care clinics for diabetes in primary care: a system-wide randomized trial. Diabetes Care, 24(4), 695-700.

Weinger, K. (2003). Group Interventions: Emerging Applications for Diabetes Care. Diabetes Spectrum, 16(2), 86-87.

Whitlock, E. P., Vogt, T. M., Hollis, J. F., \& Lichtenstein, E. (1997). Does gender affect response to a brief clinic-based smoking intervention? Am J Prev Med, 13(3), 159-166. 
Appendix A: Q-Q plots for differences in means of biophysical outcomes Aim 3
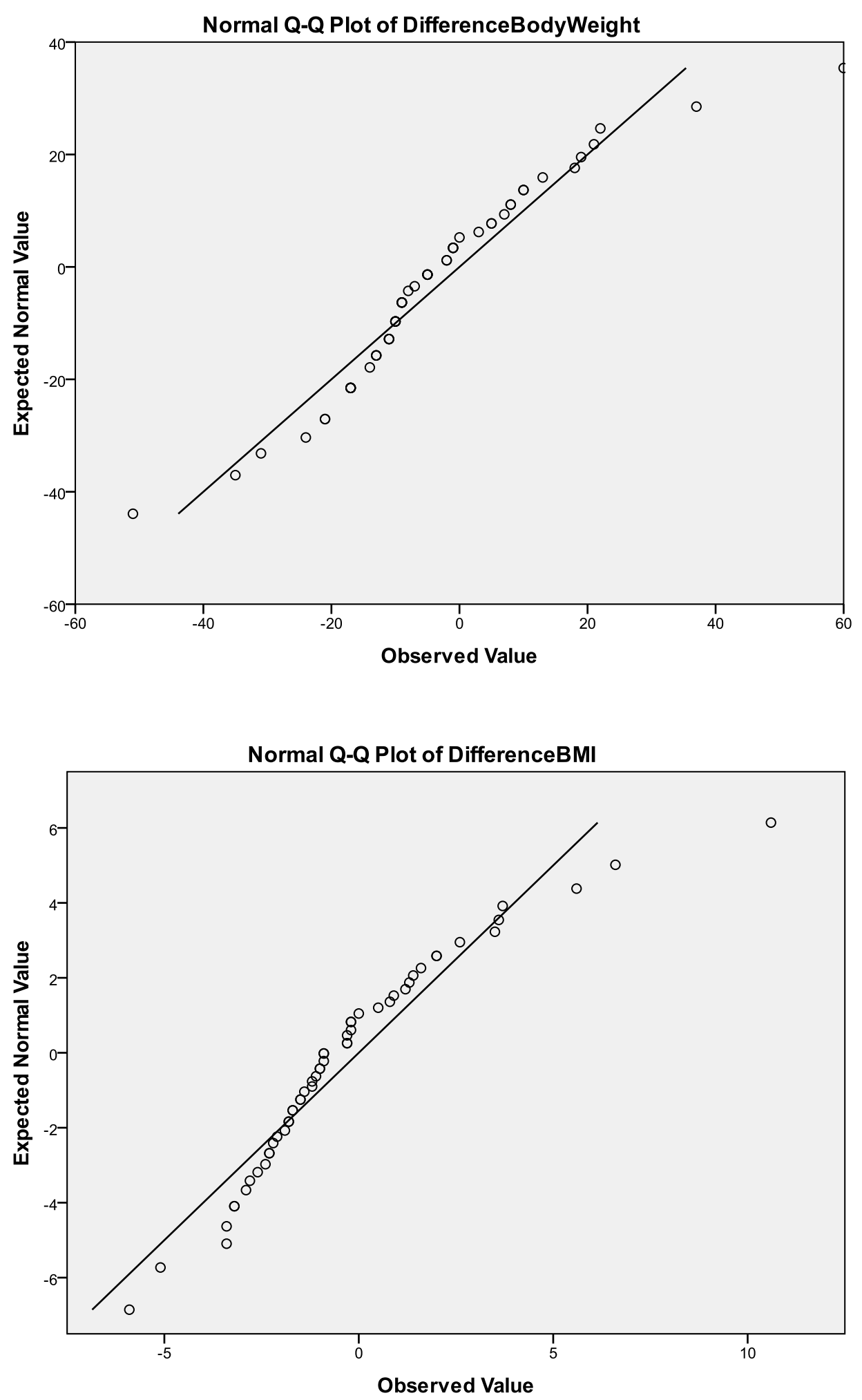

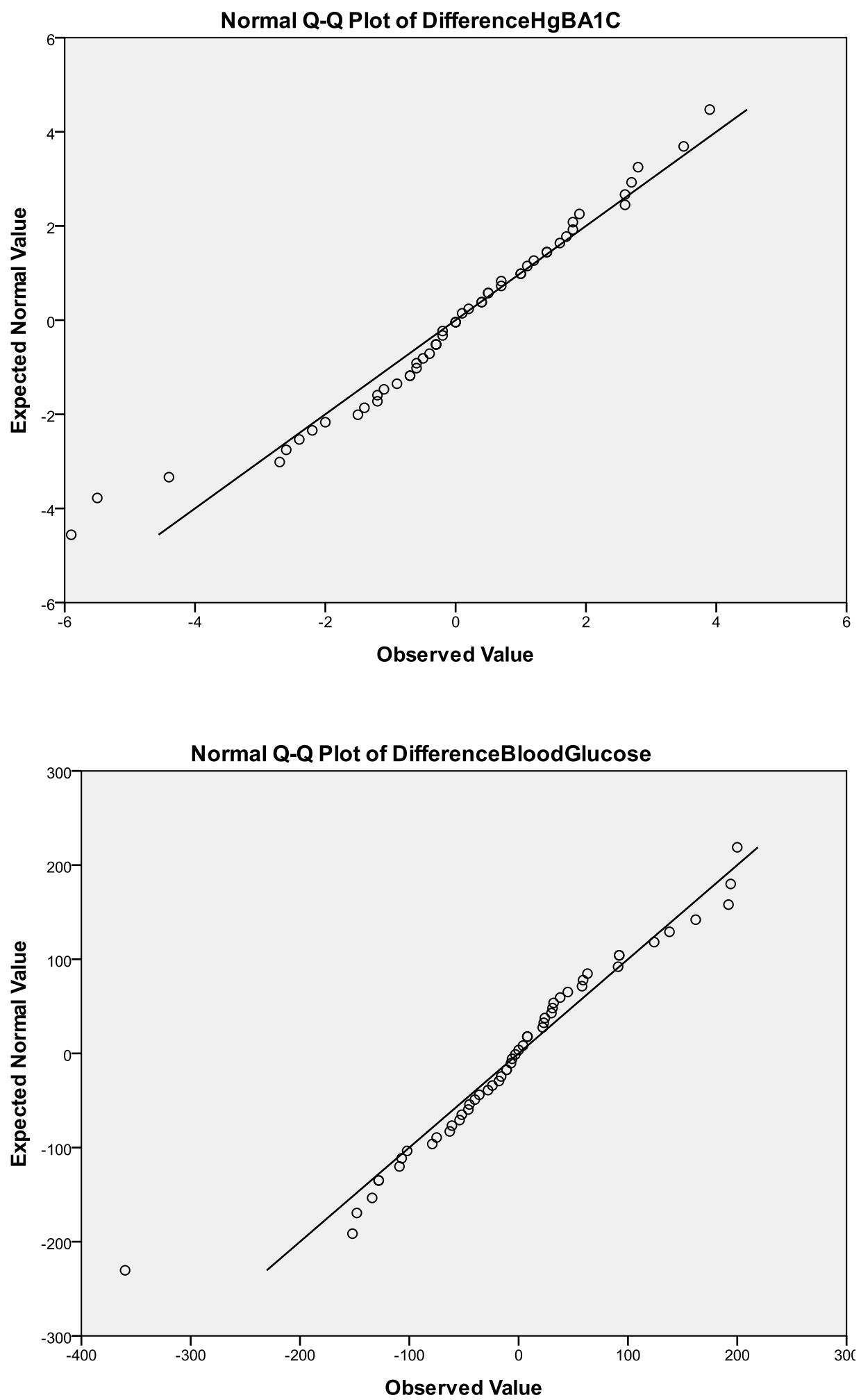

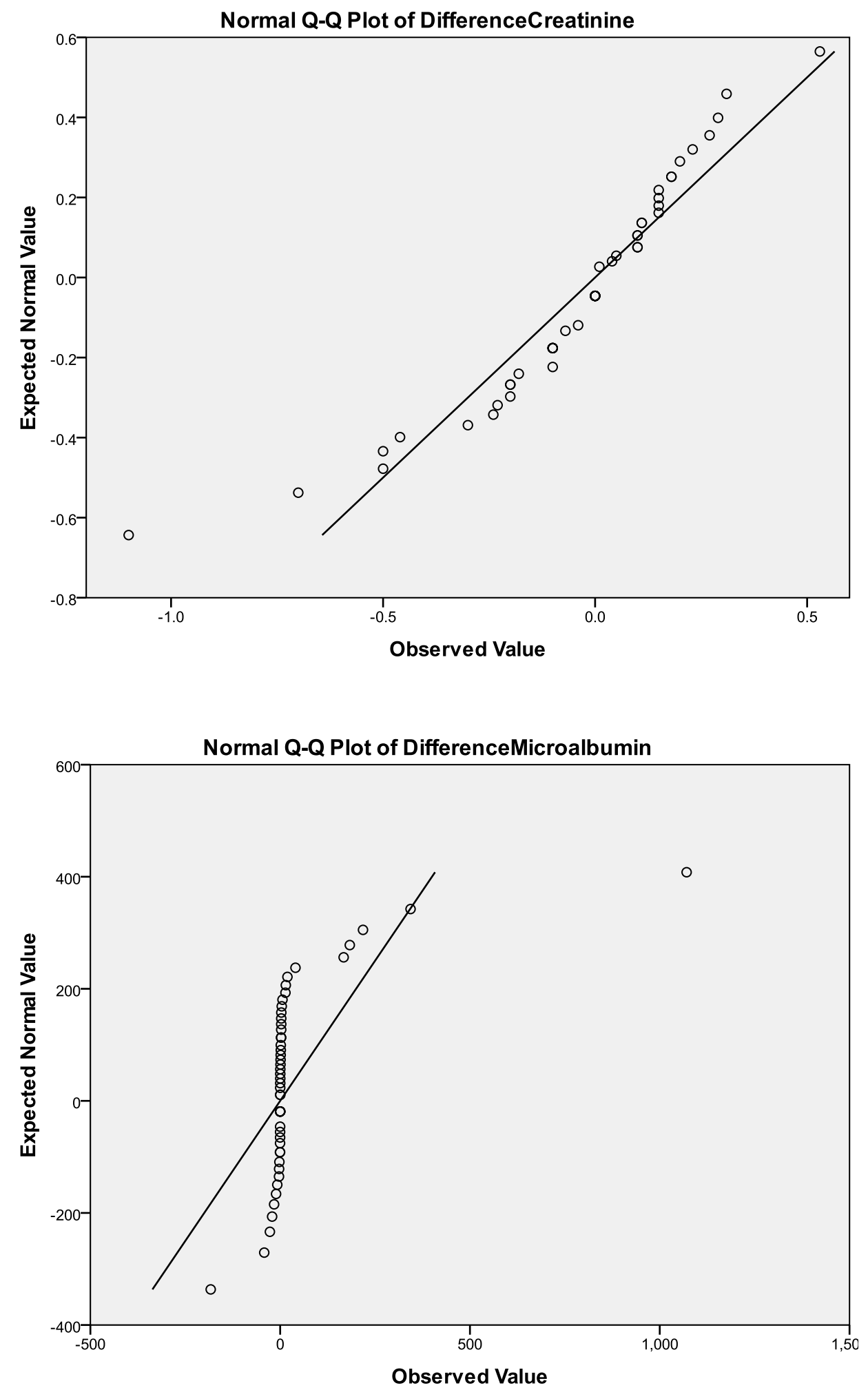

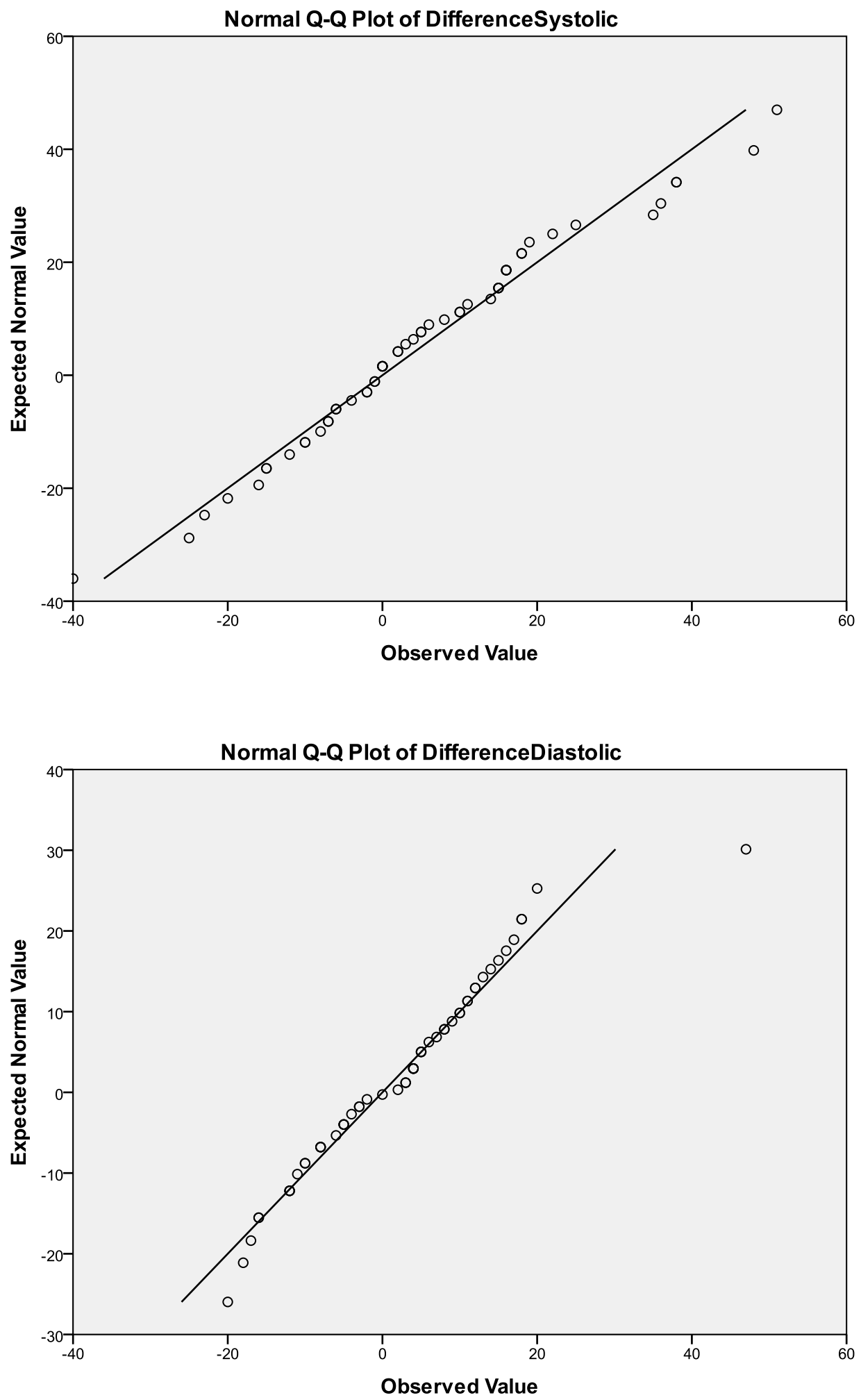

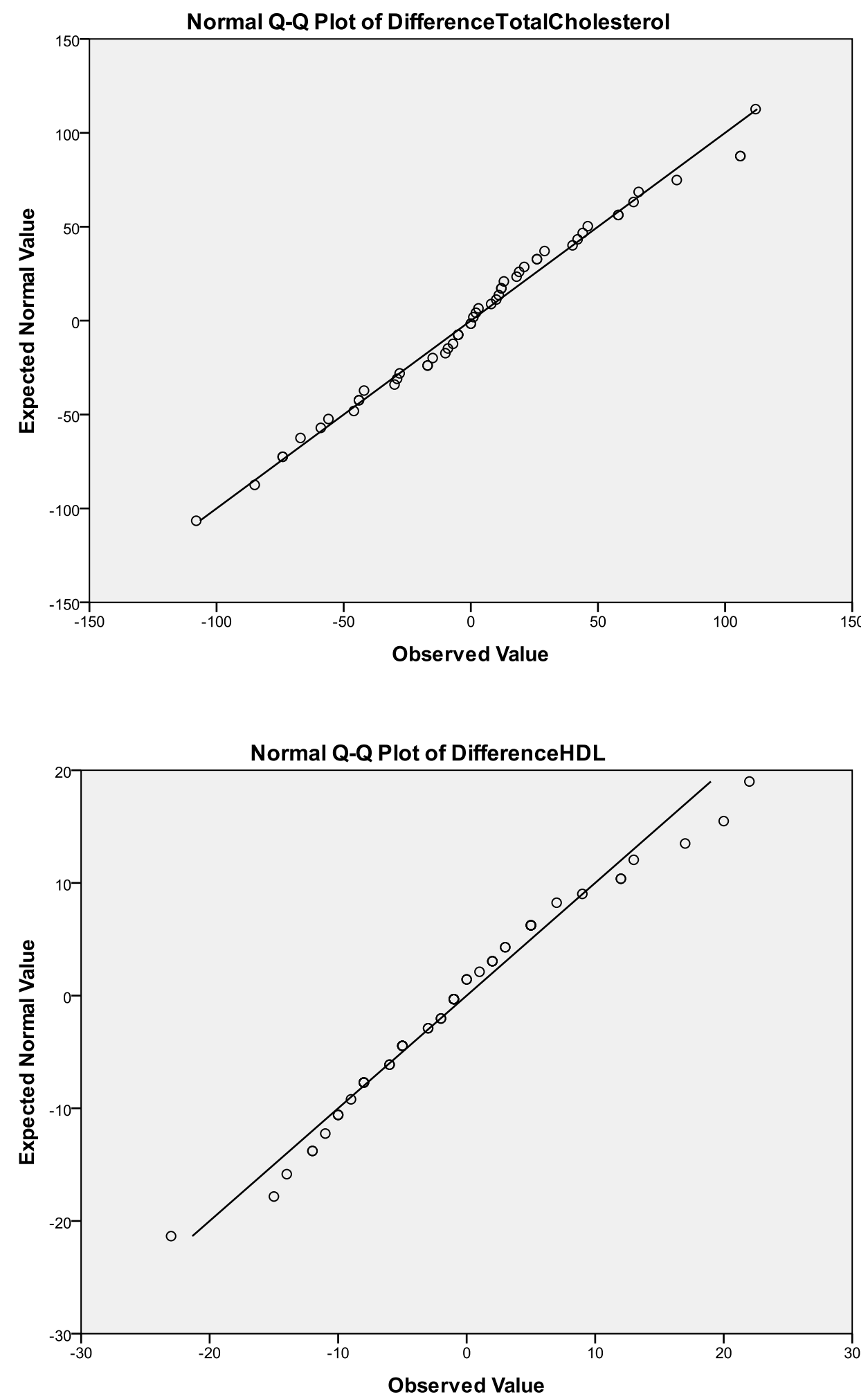

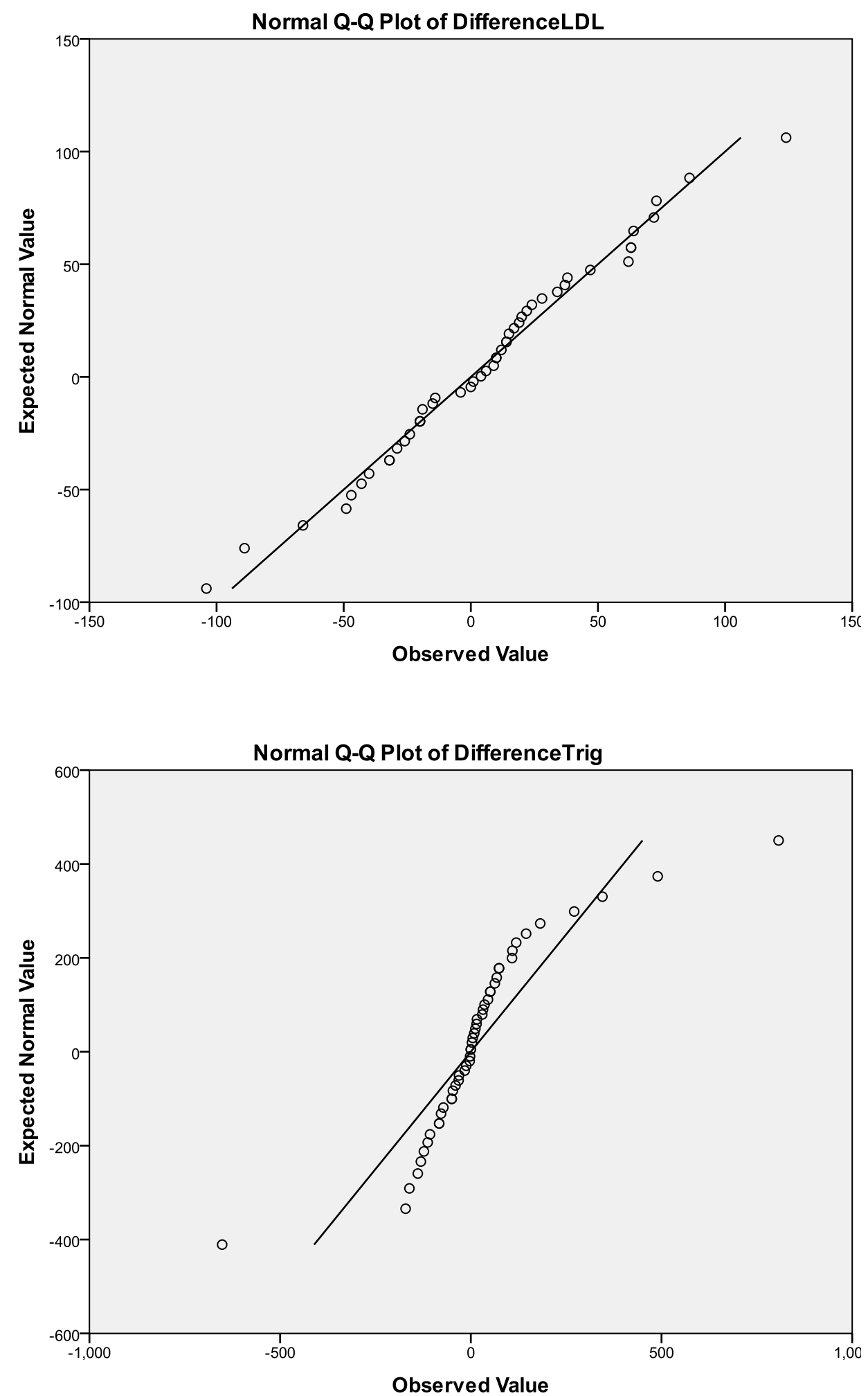


\section{Appendix B: Q-Q plots Aim 4}
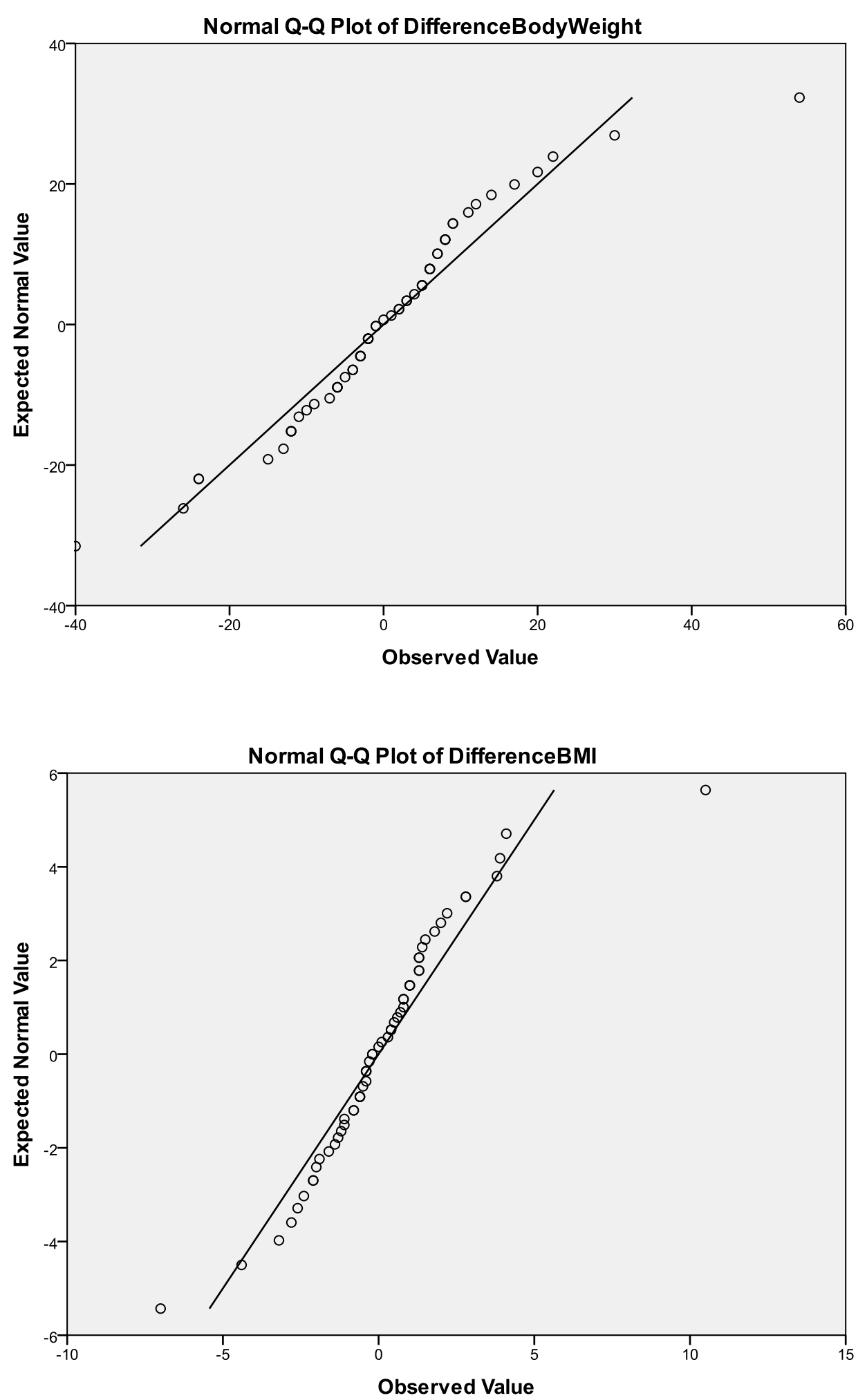

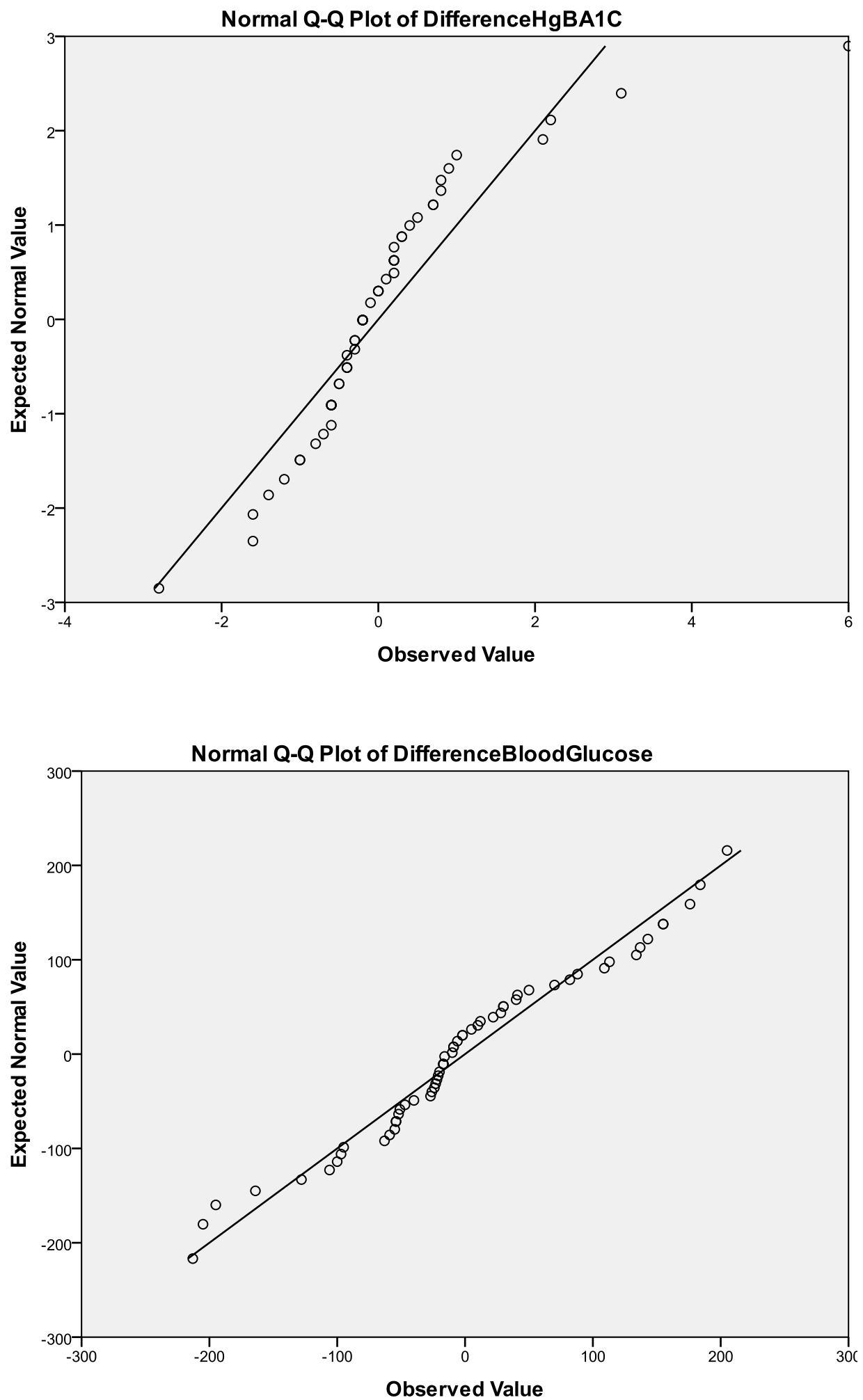

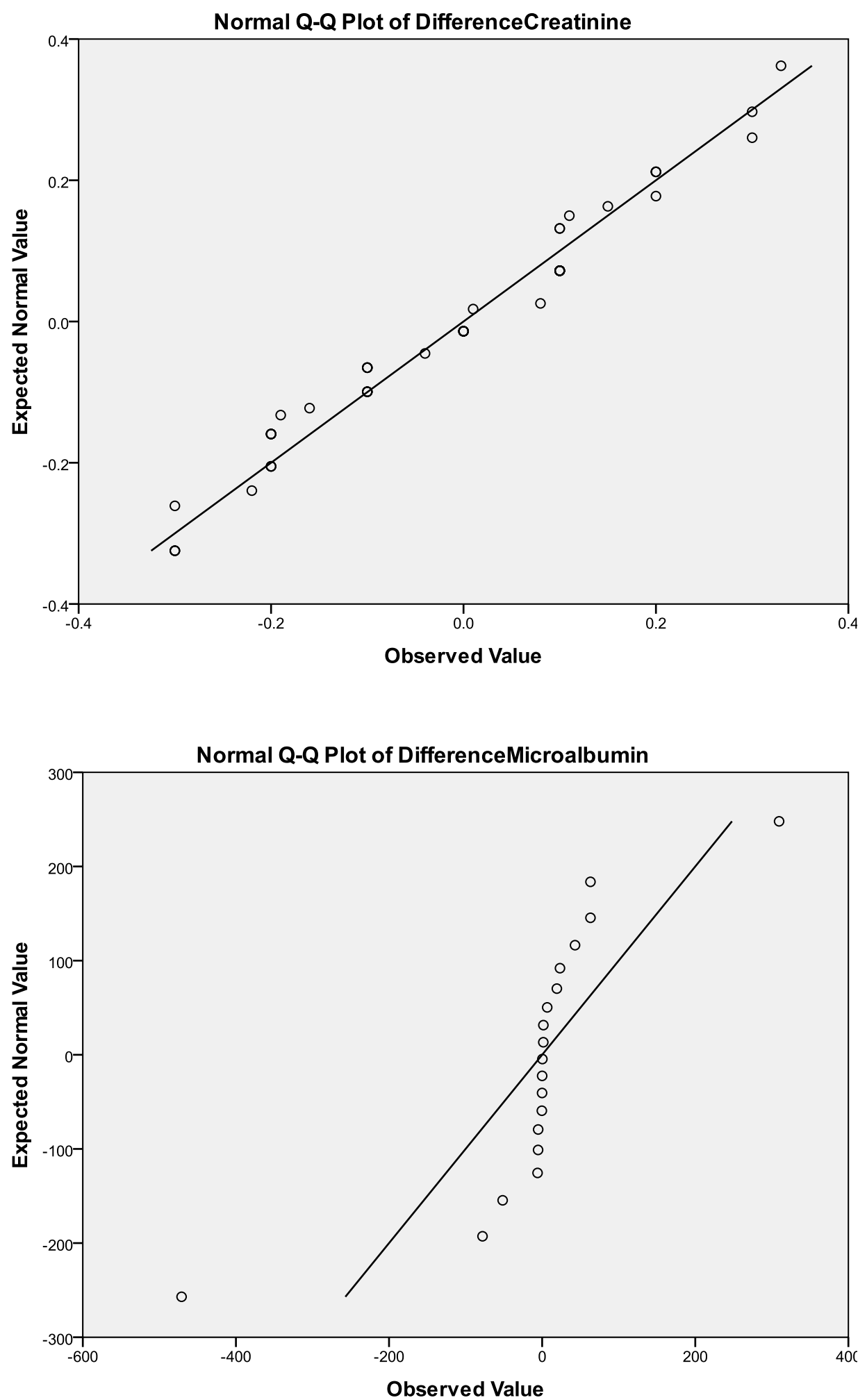

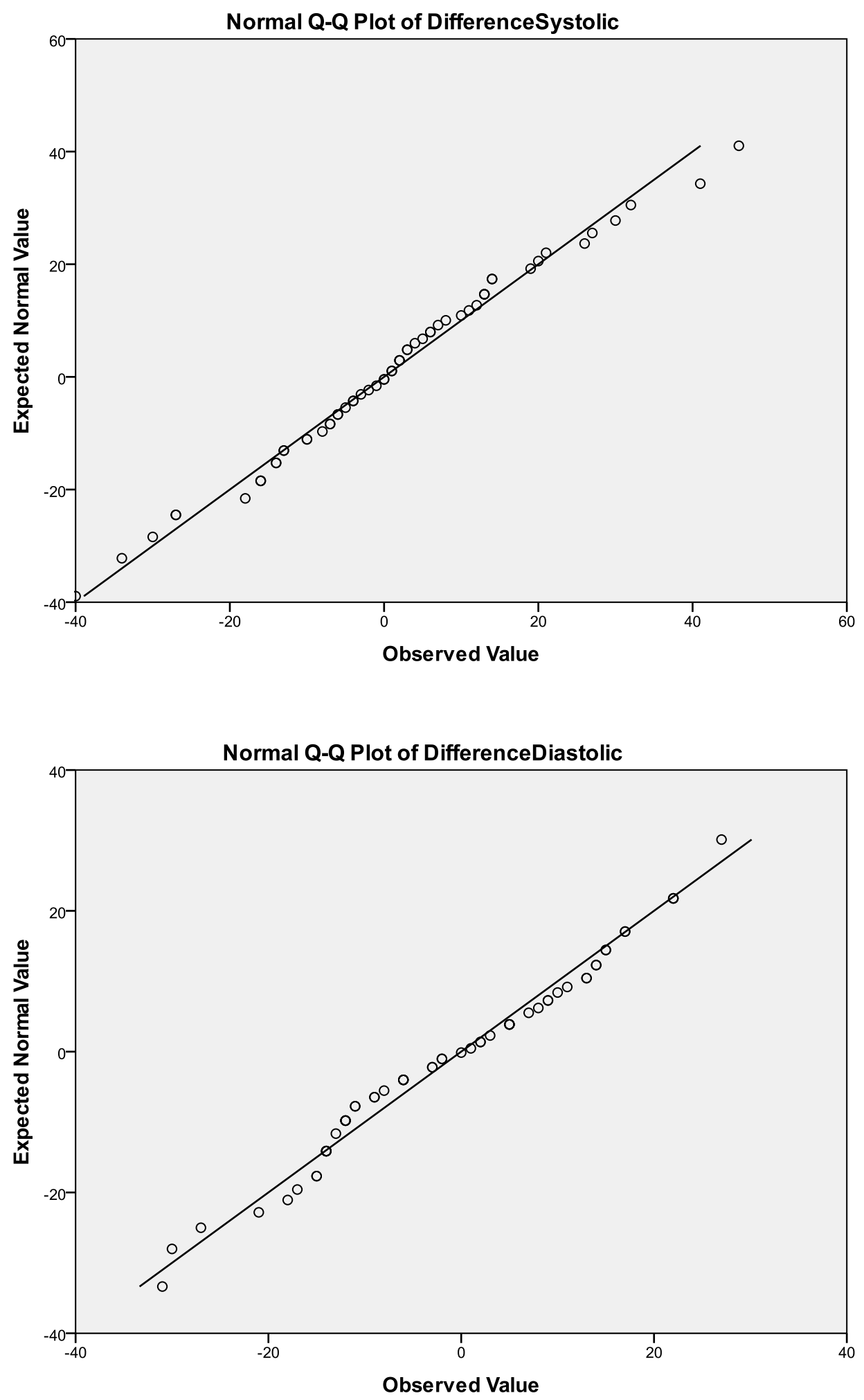

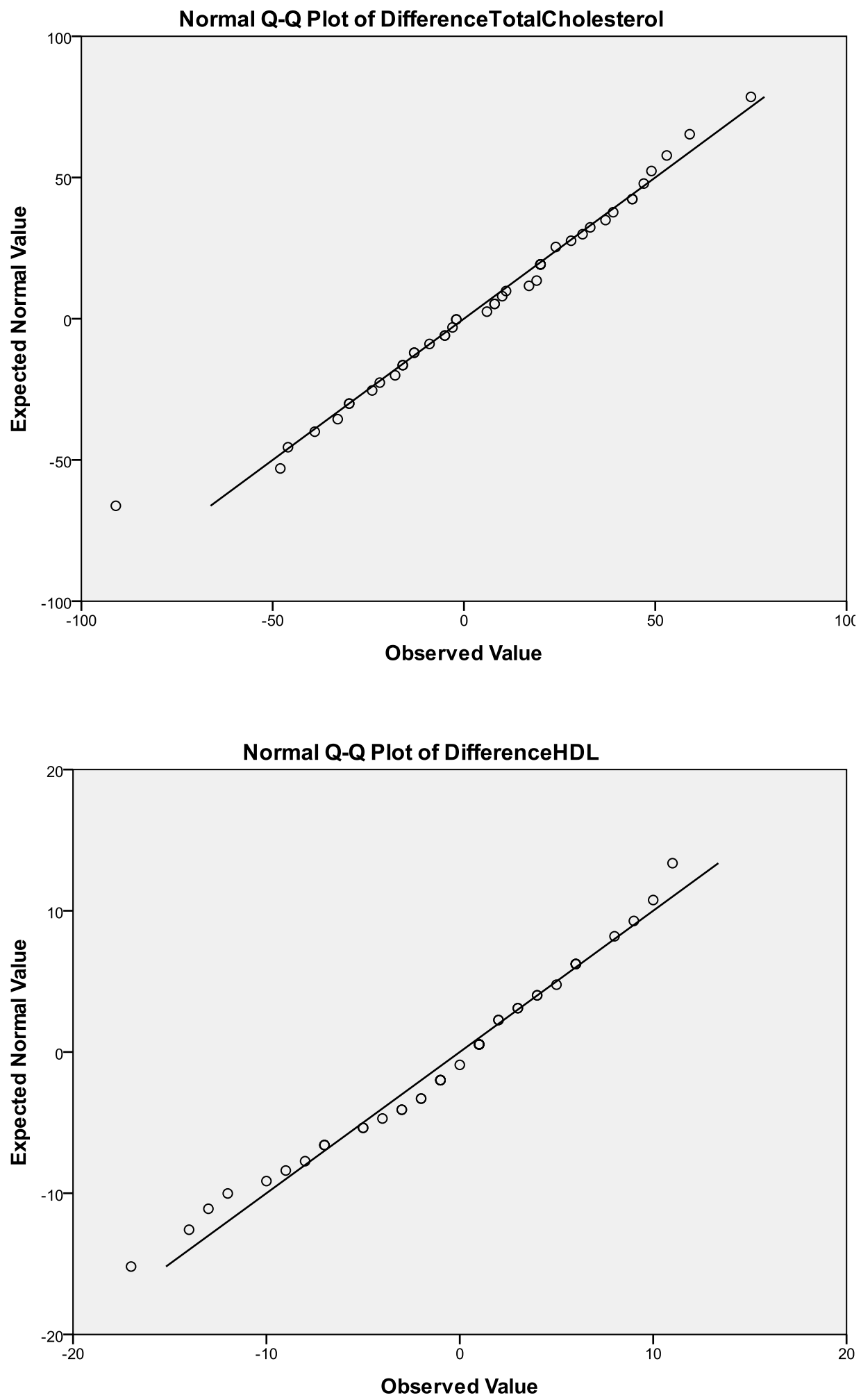

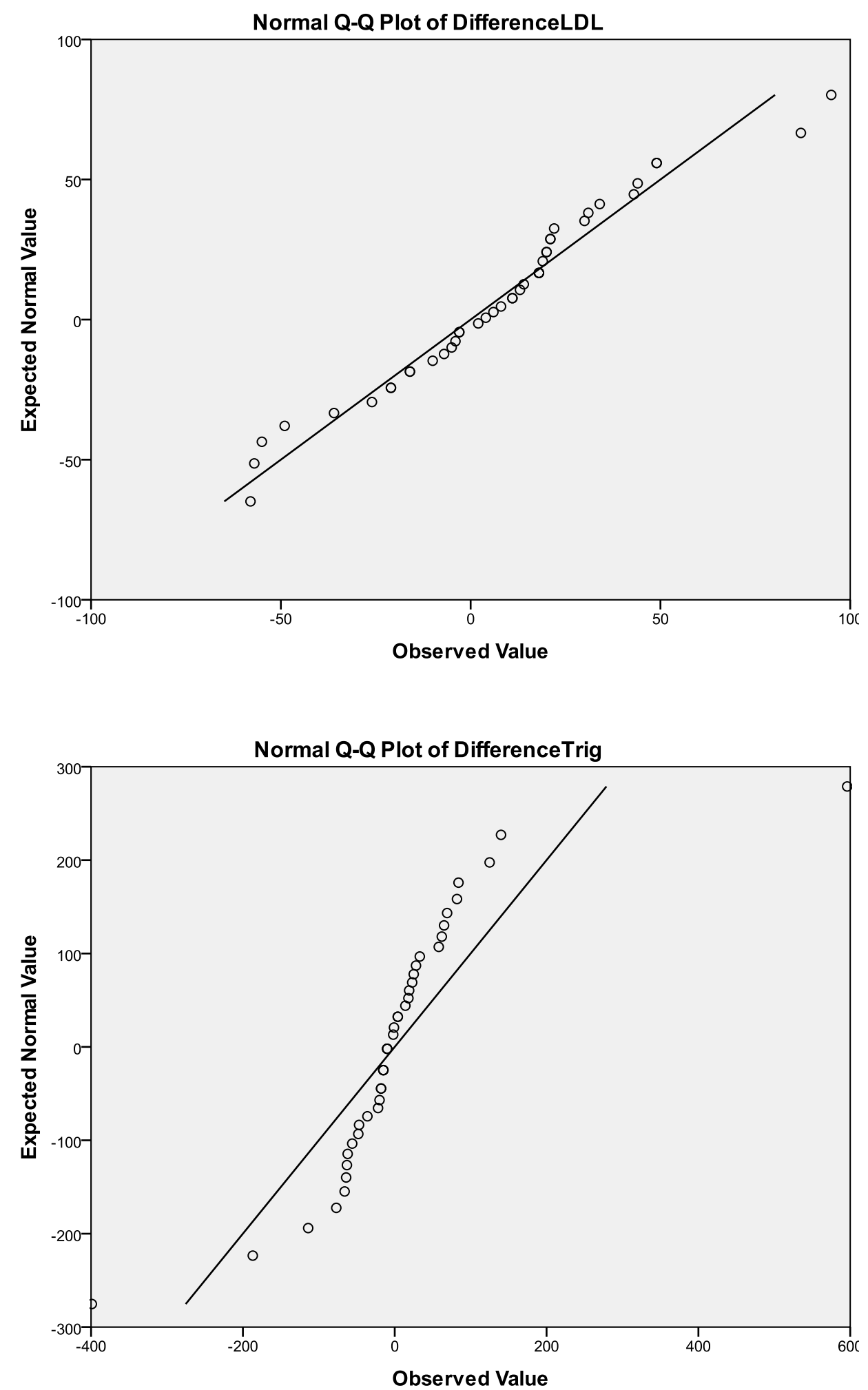


\section{Appendix C: Q-Q plots for Aim 5 Usual Care}
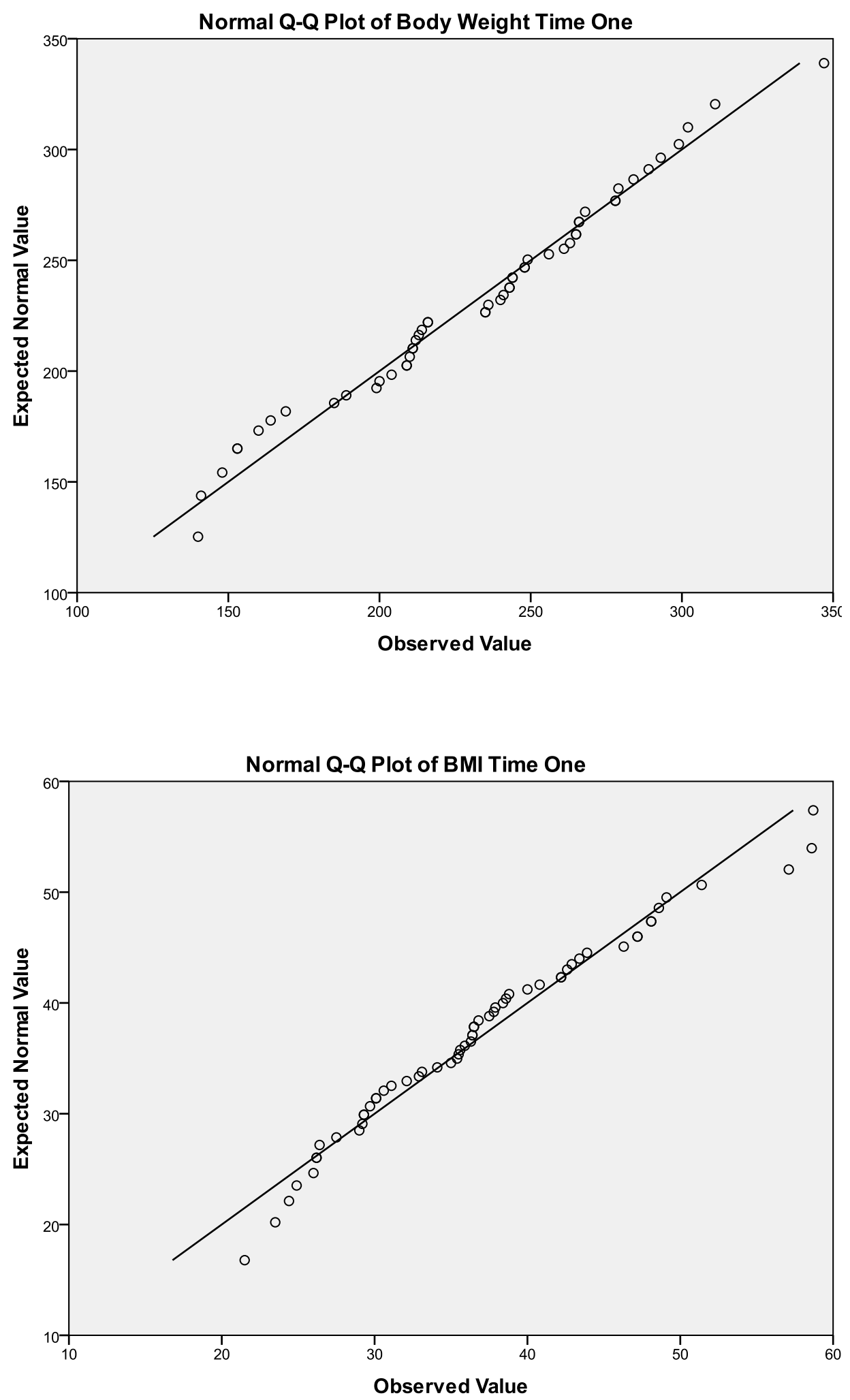

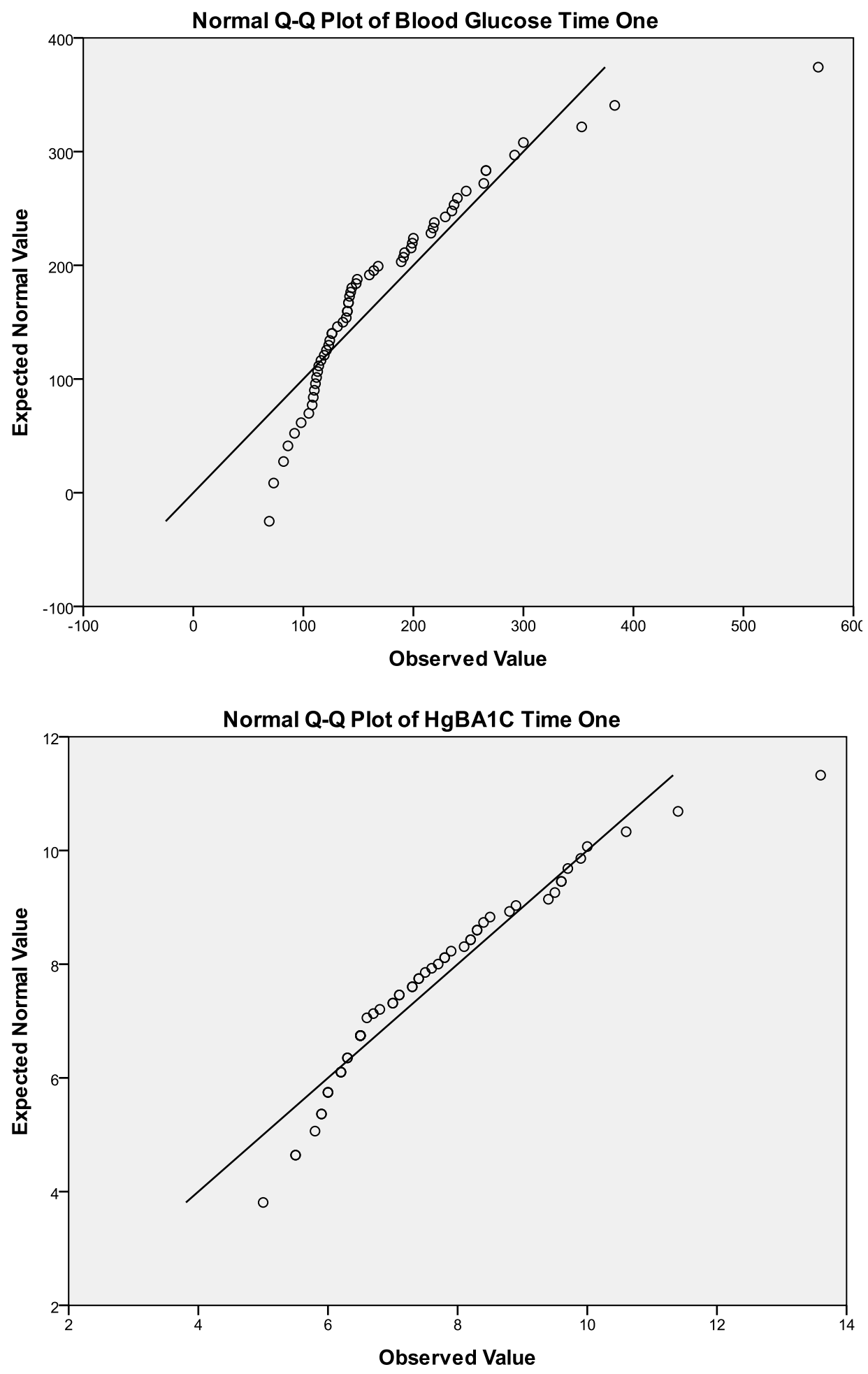

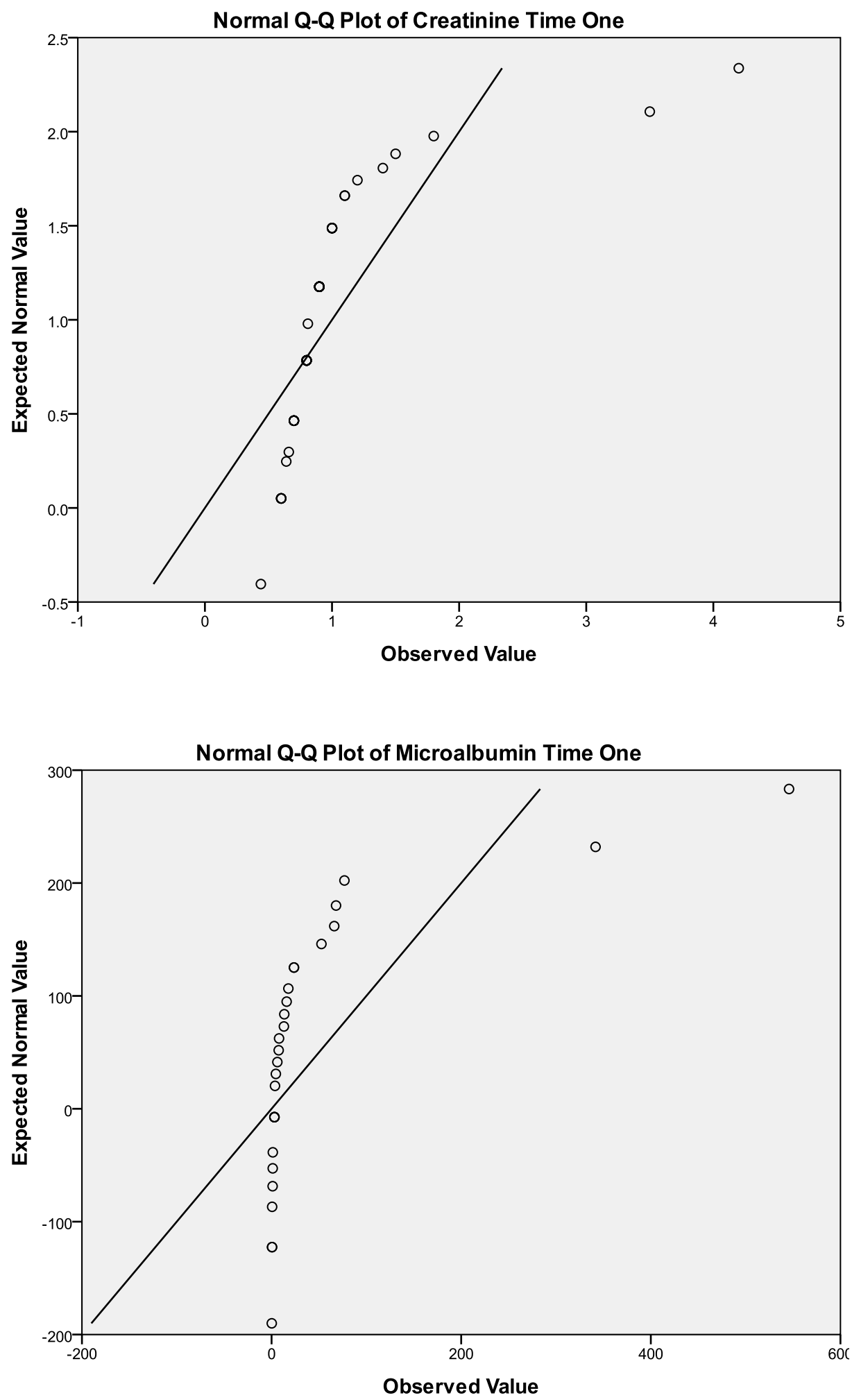

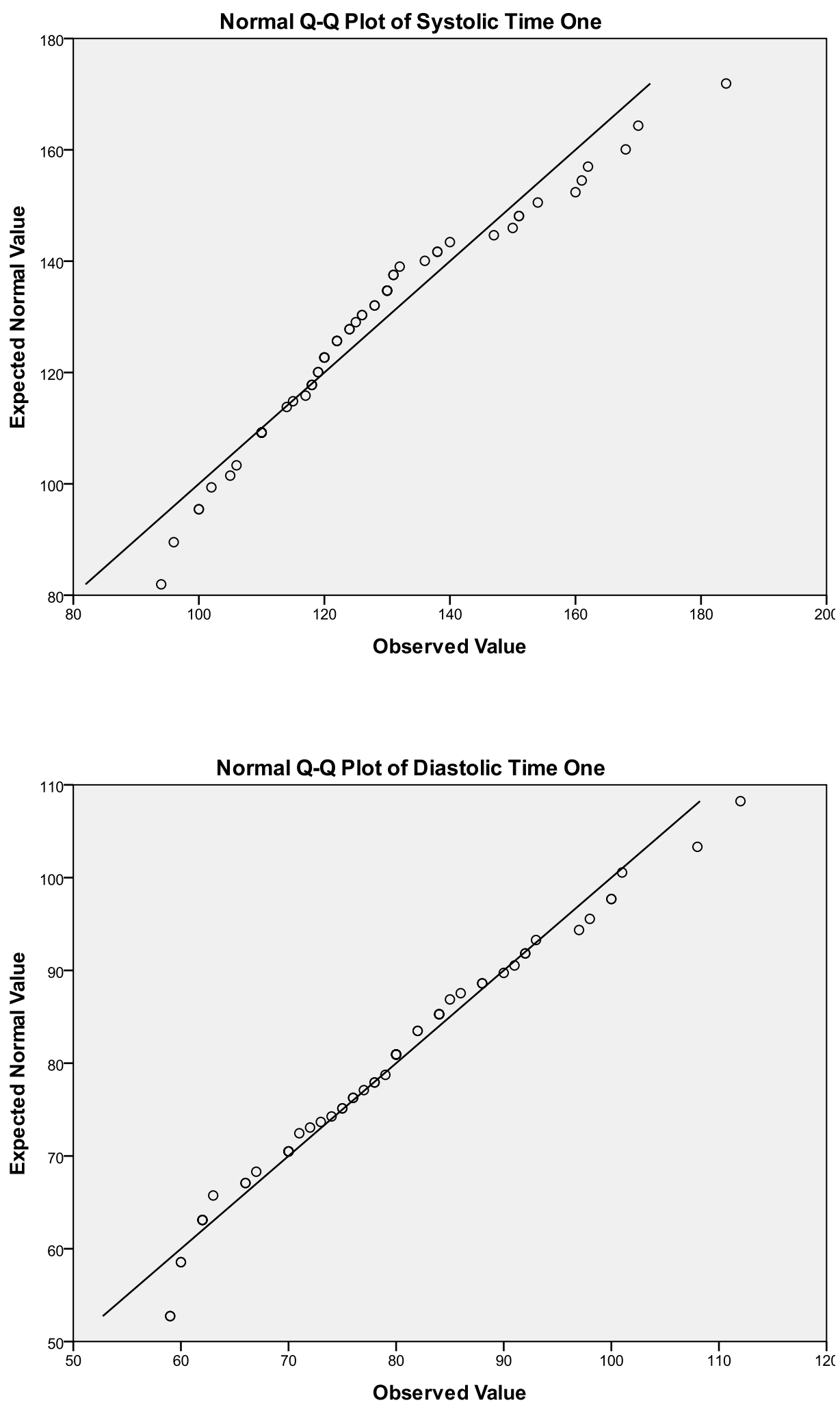

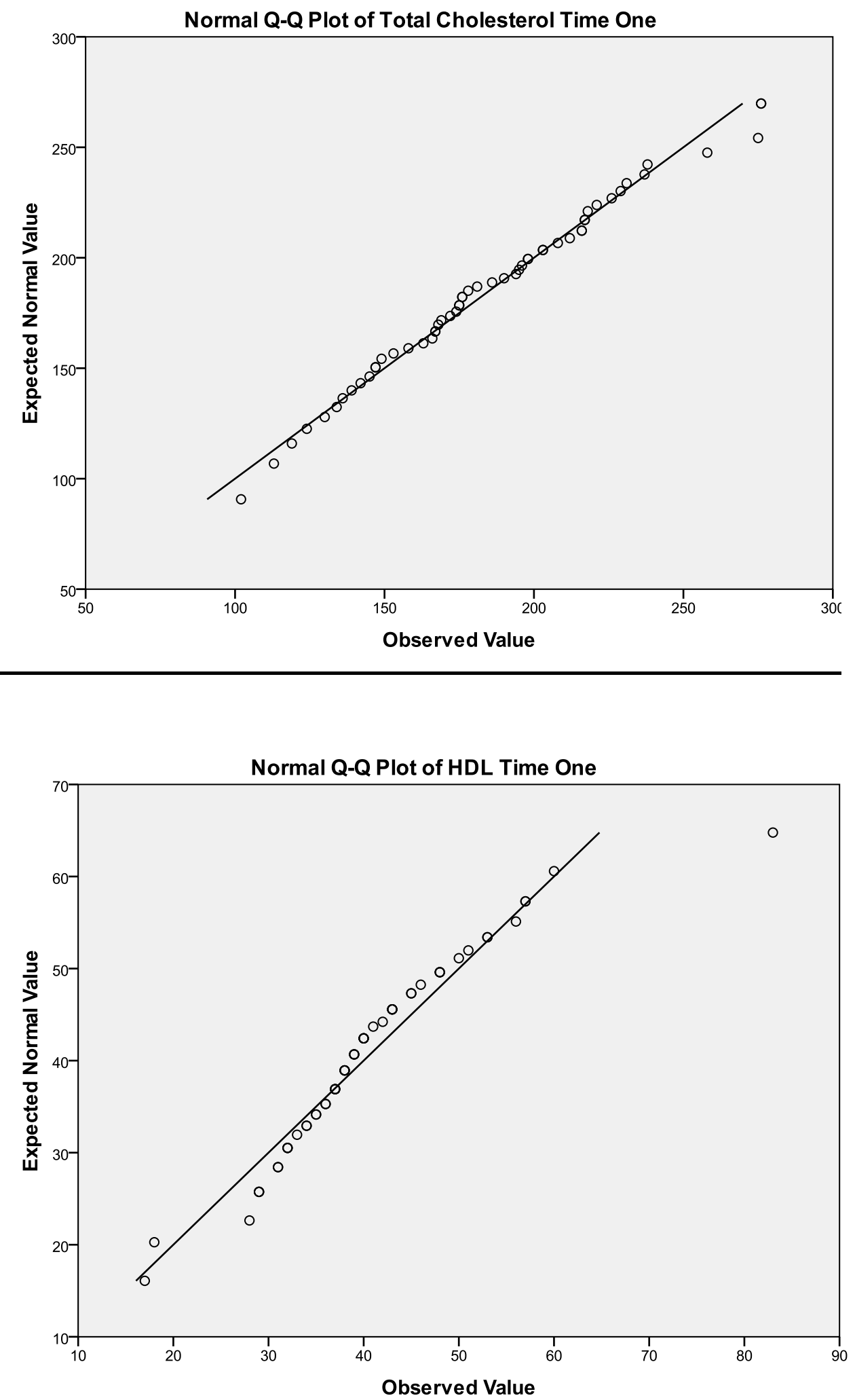

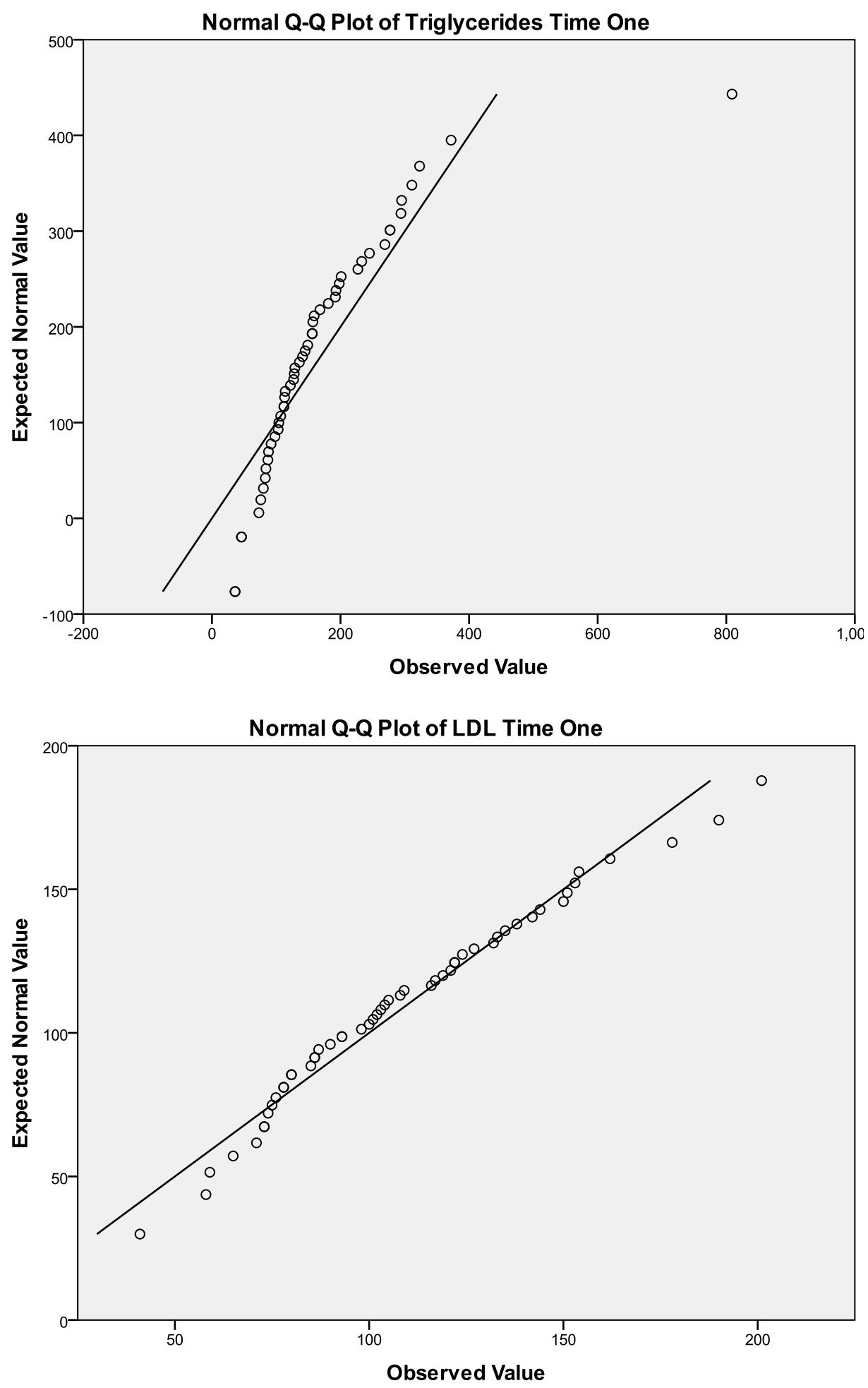

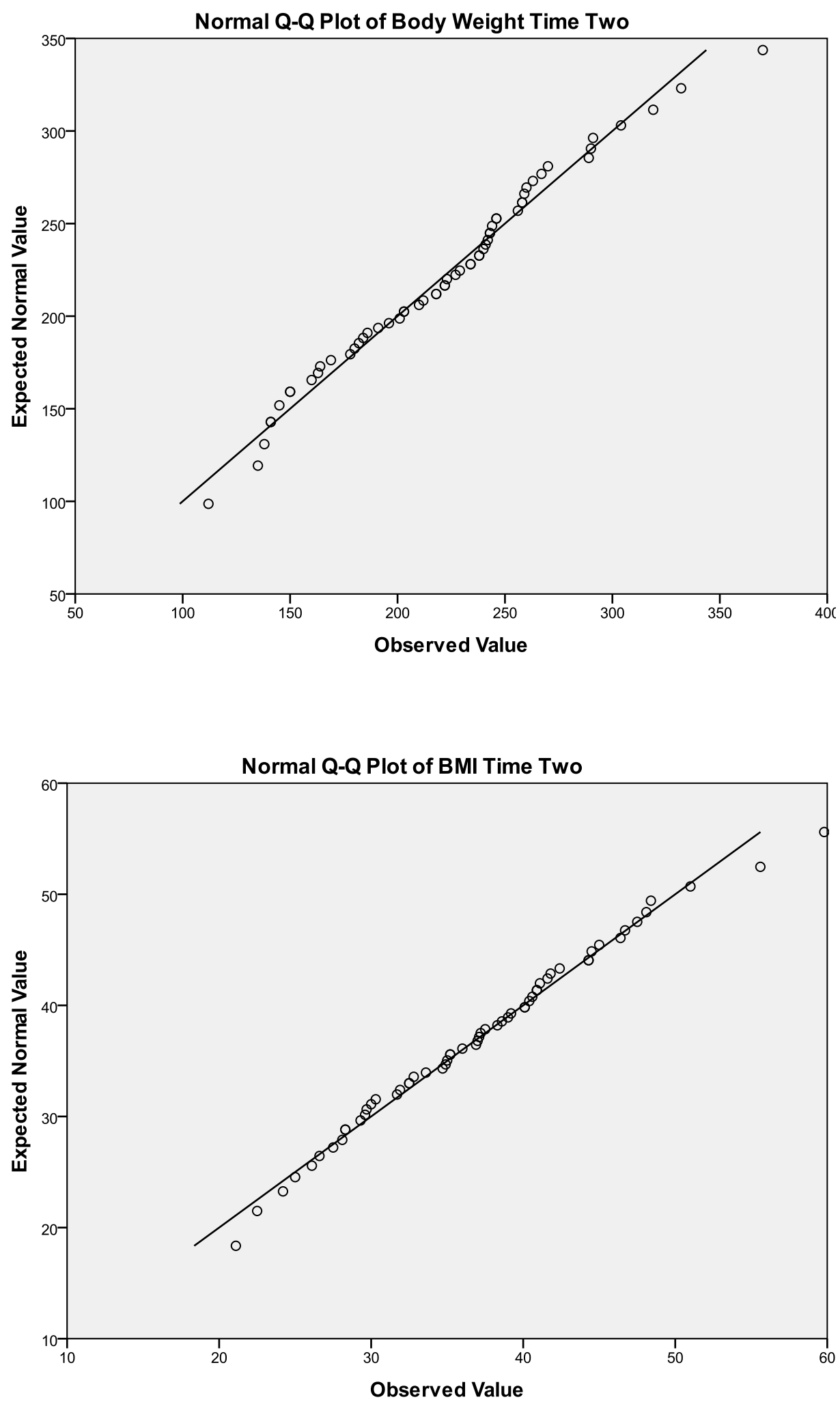

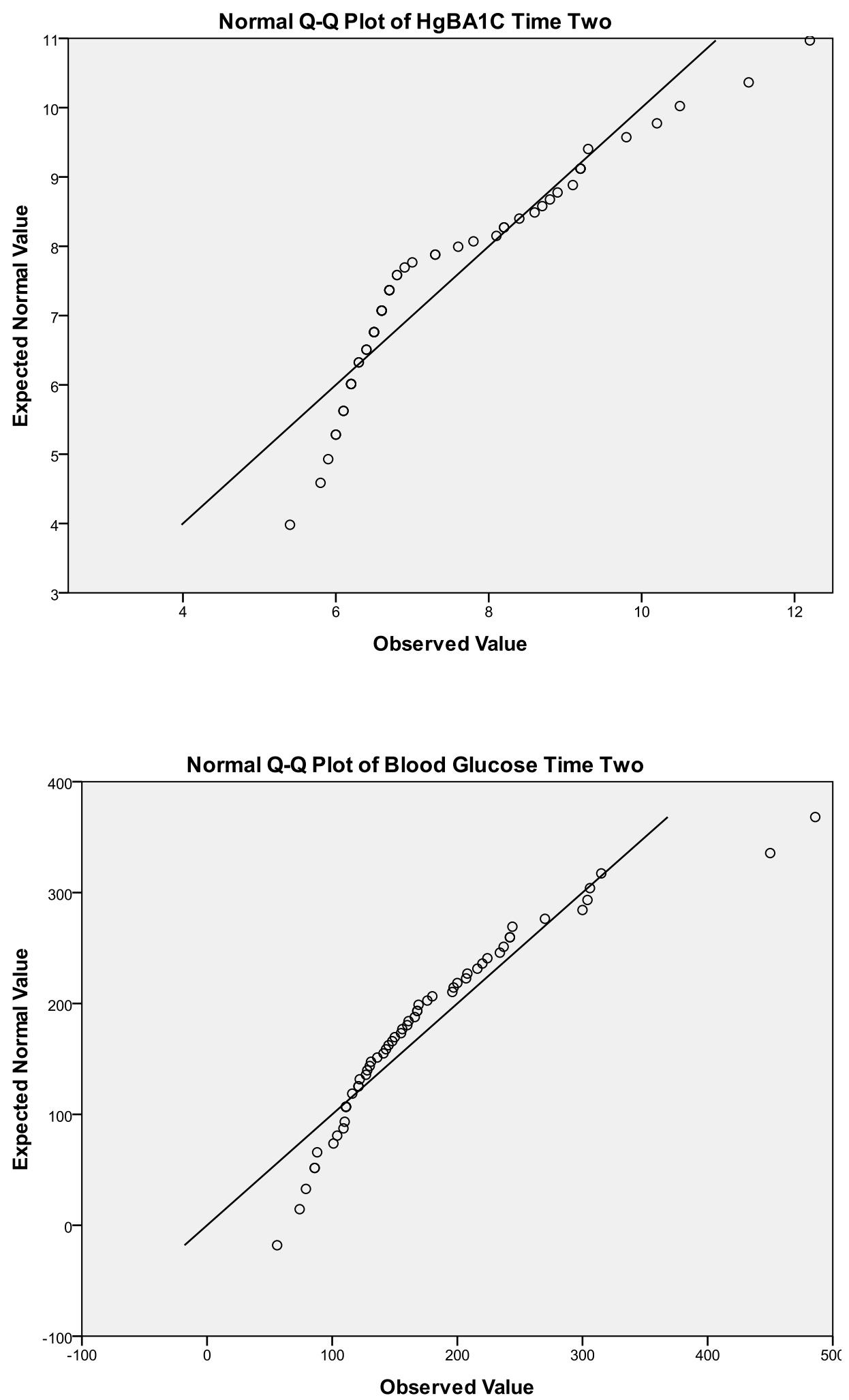

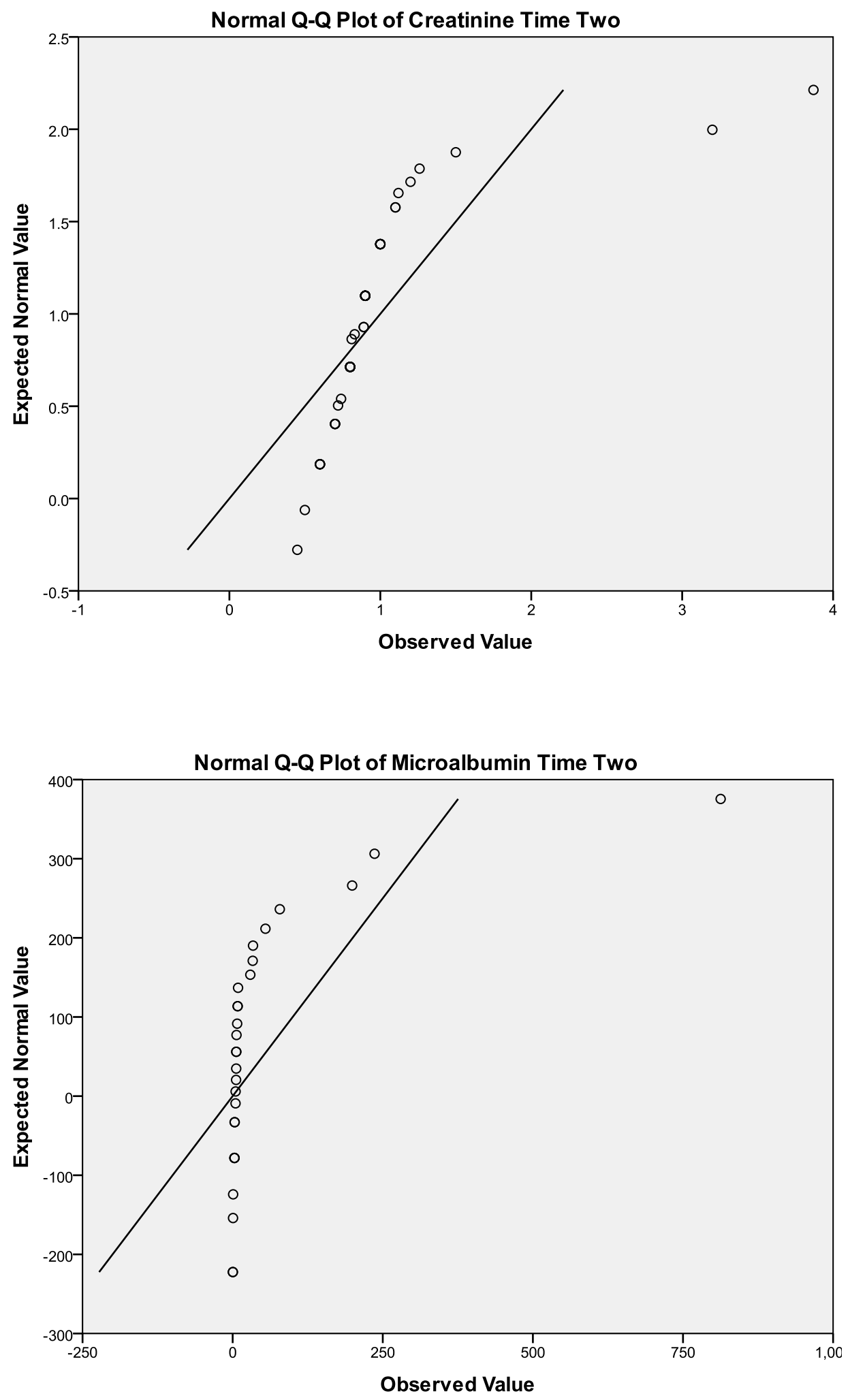

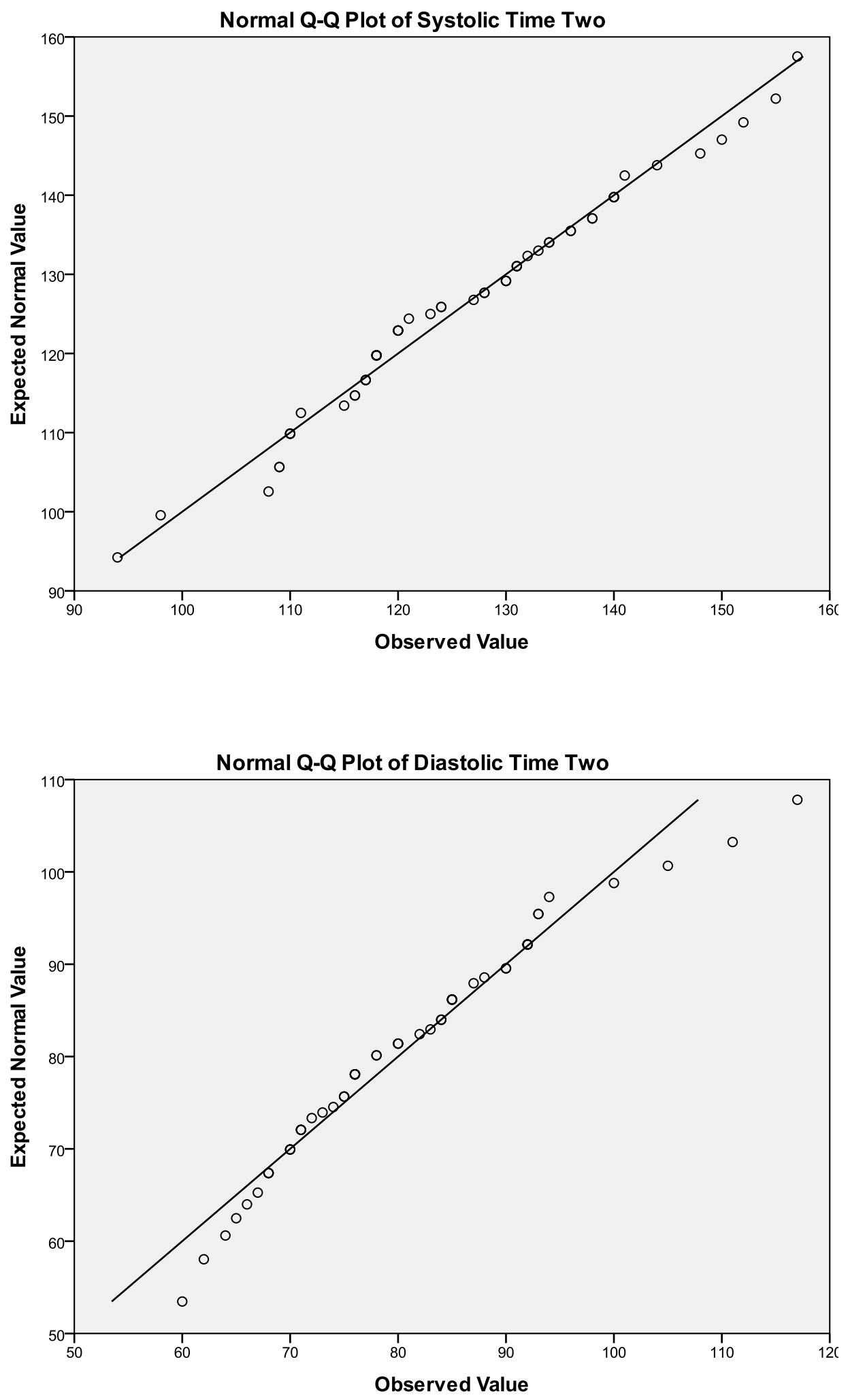

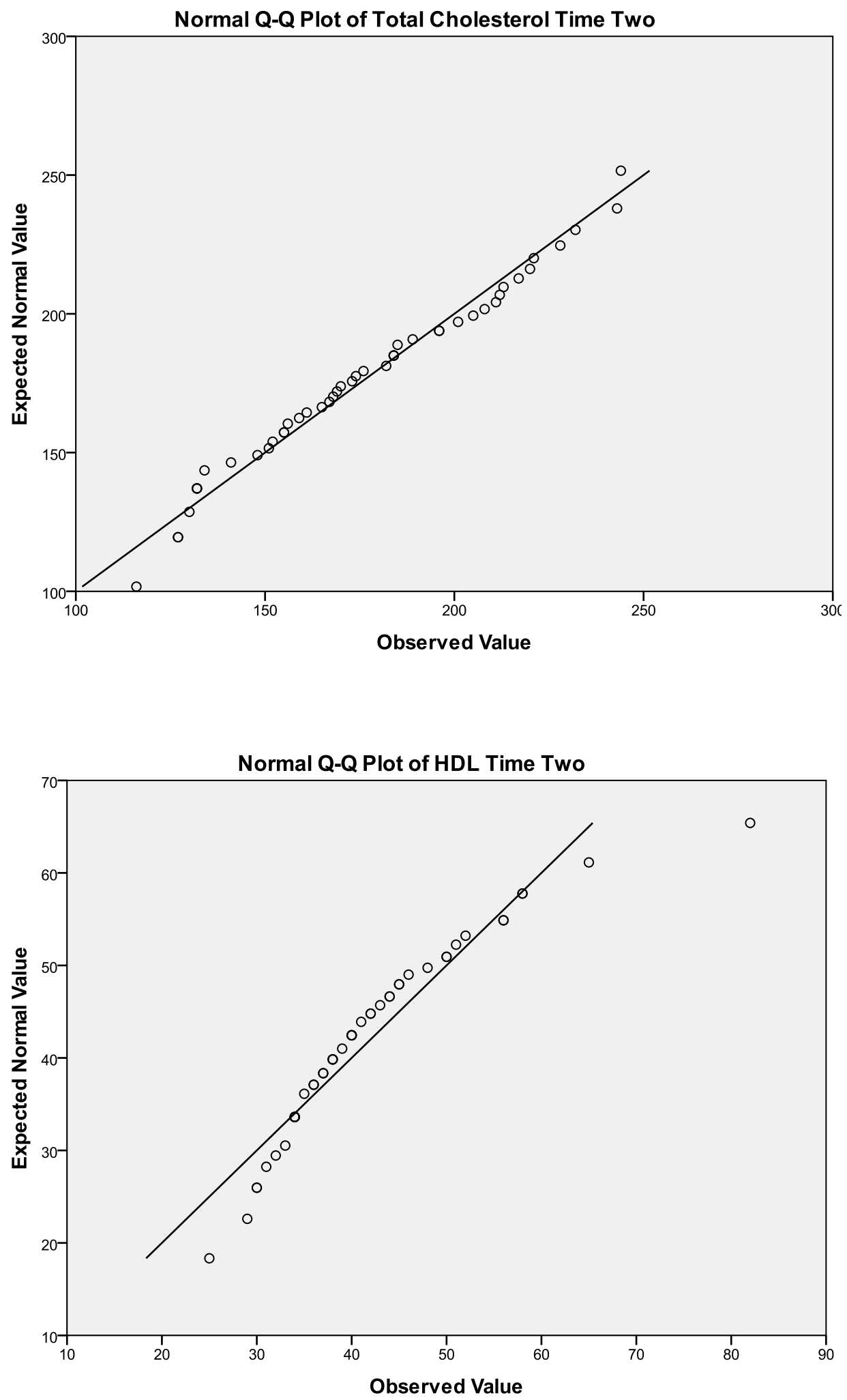

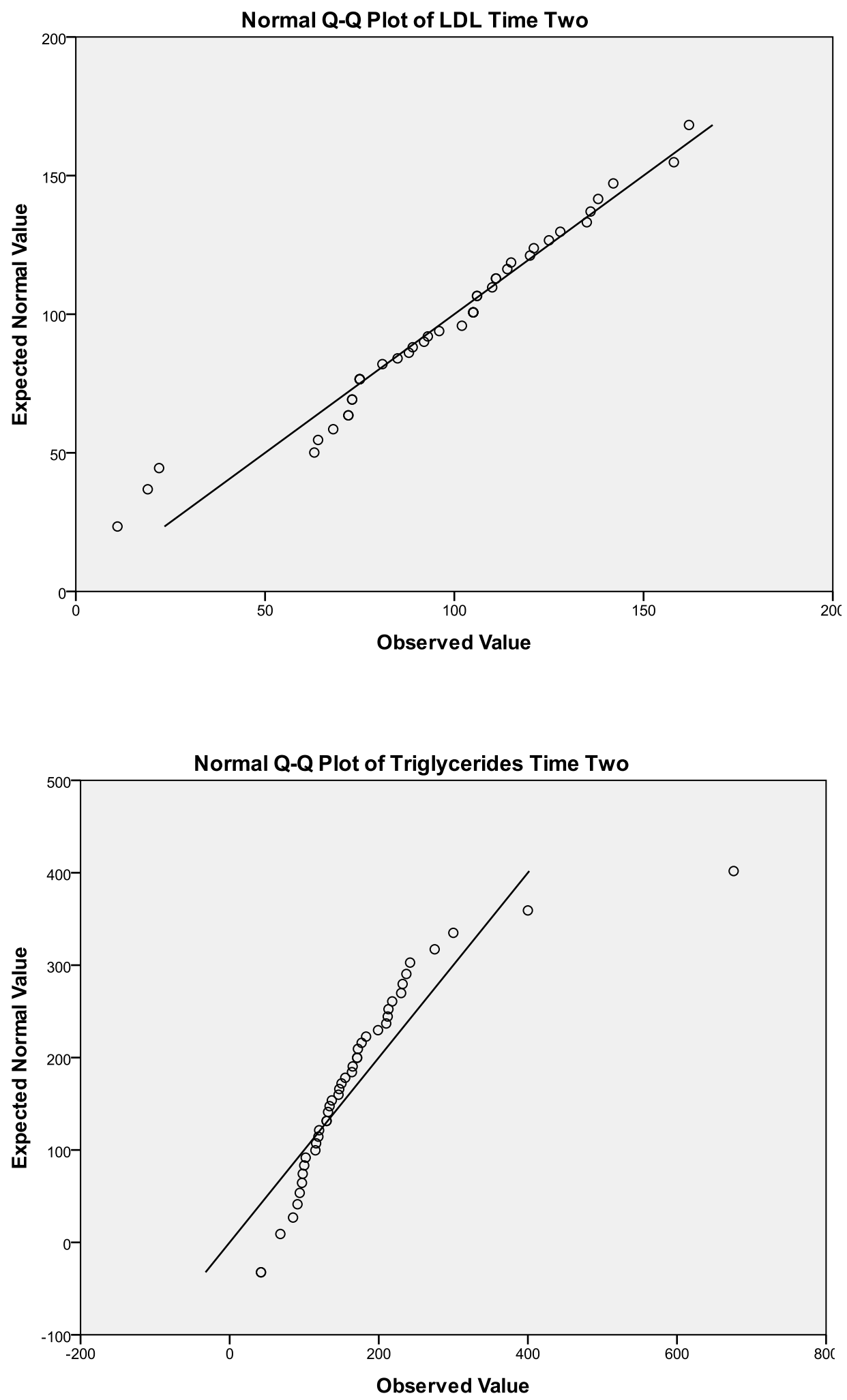


\section{Appendix D: Q-Q plots for Aim 5 DGMVs}
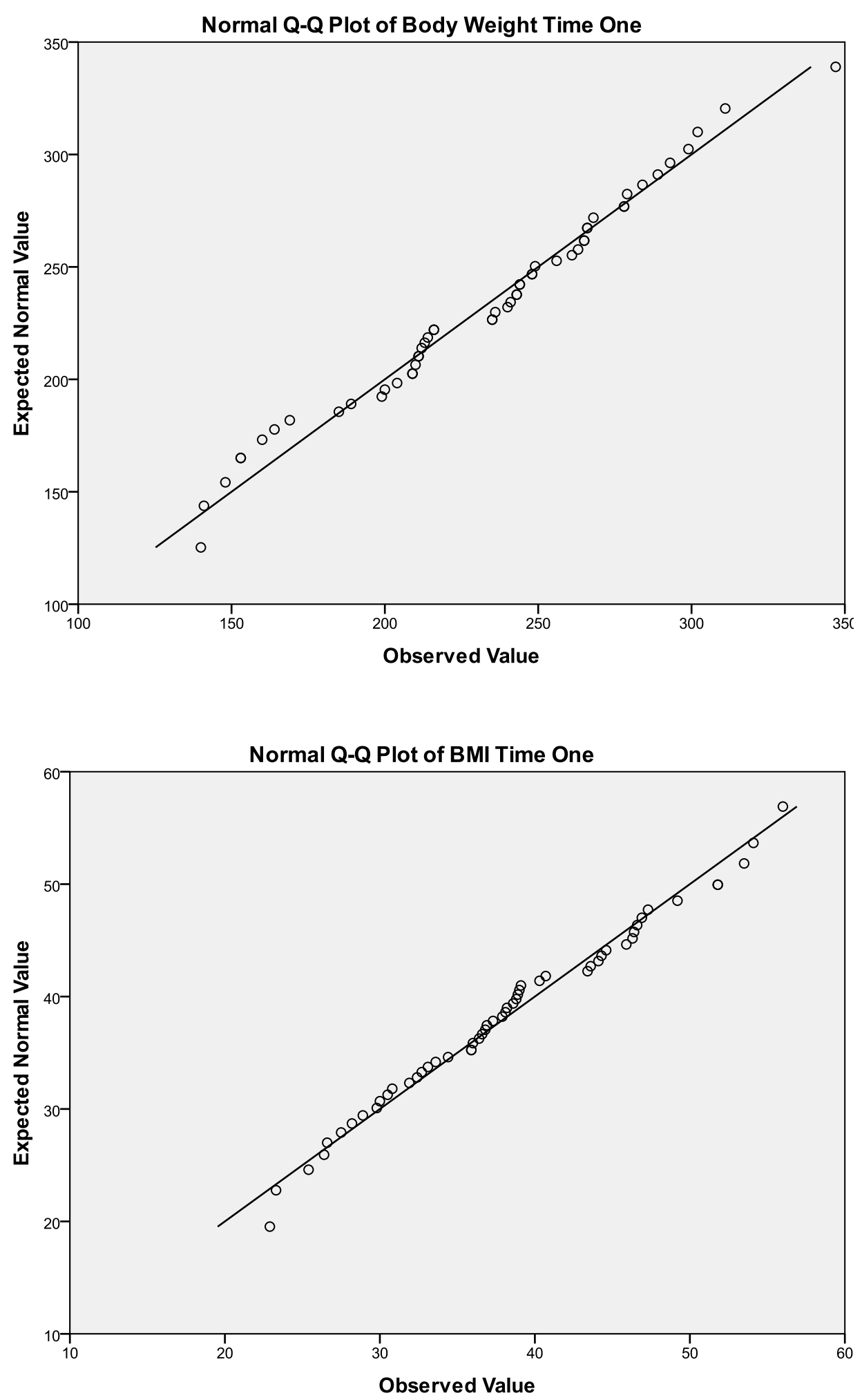

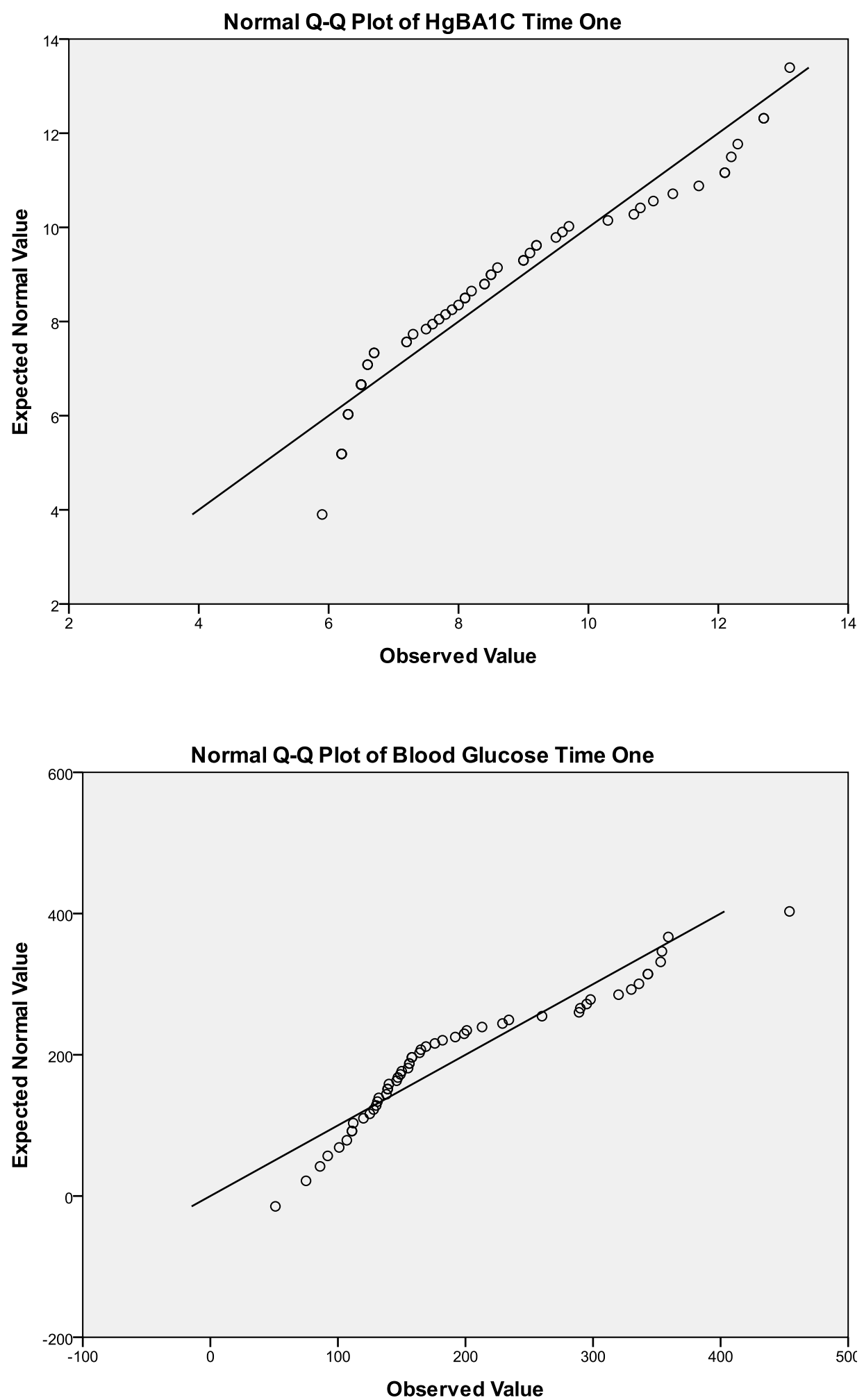

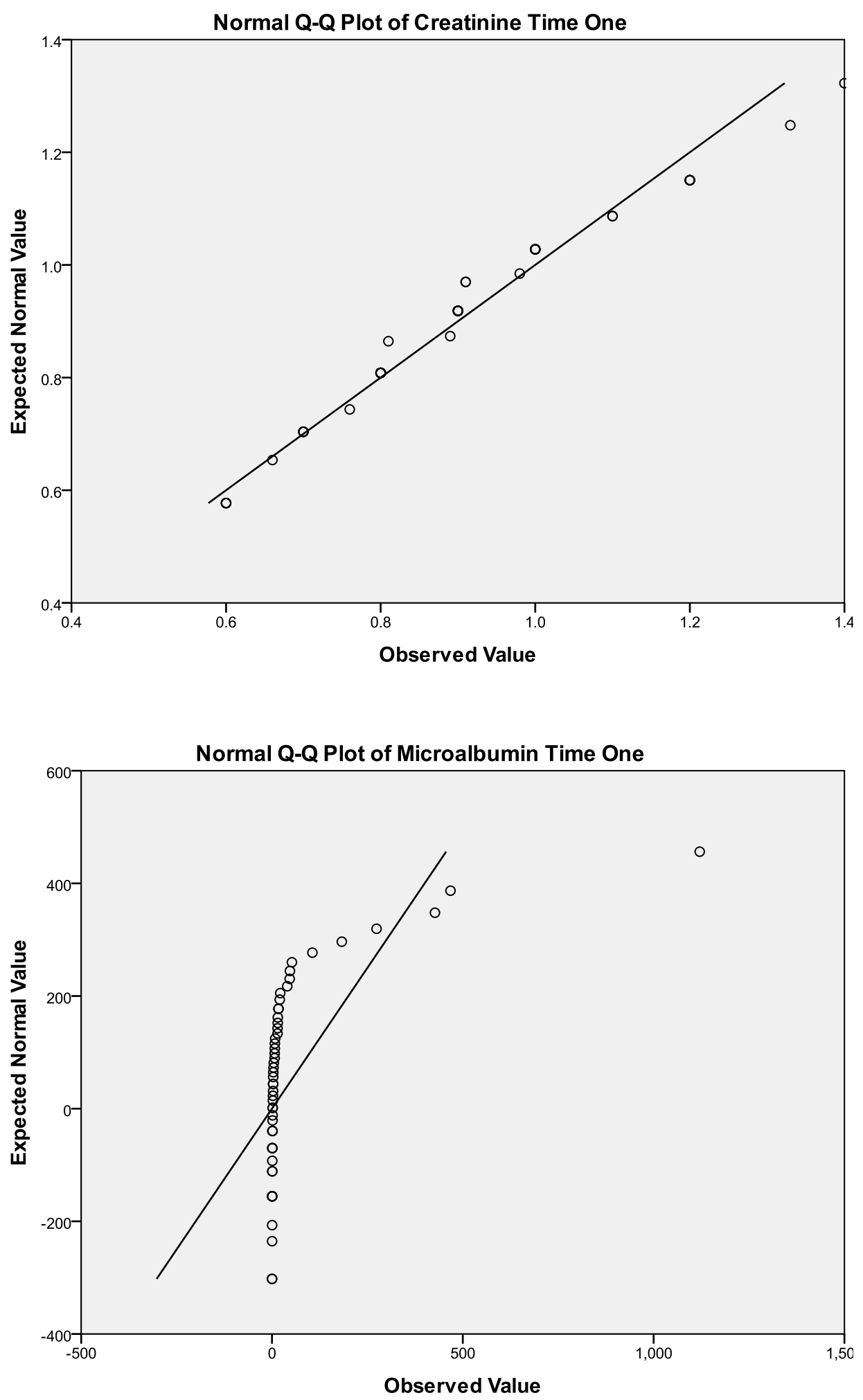

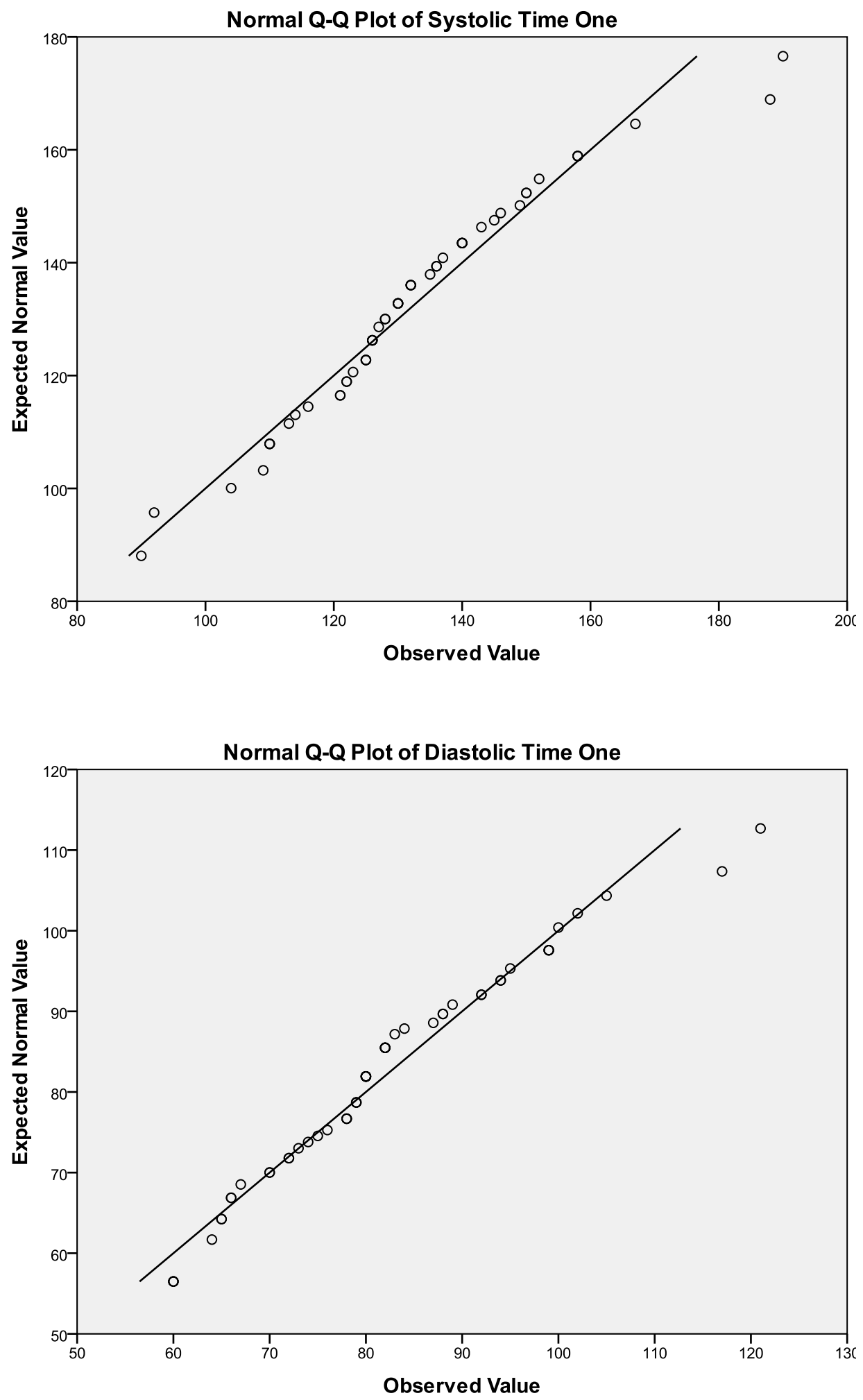

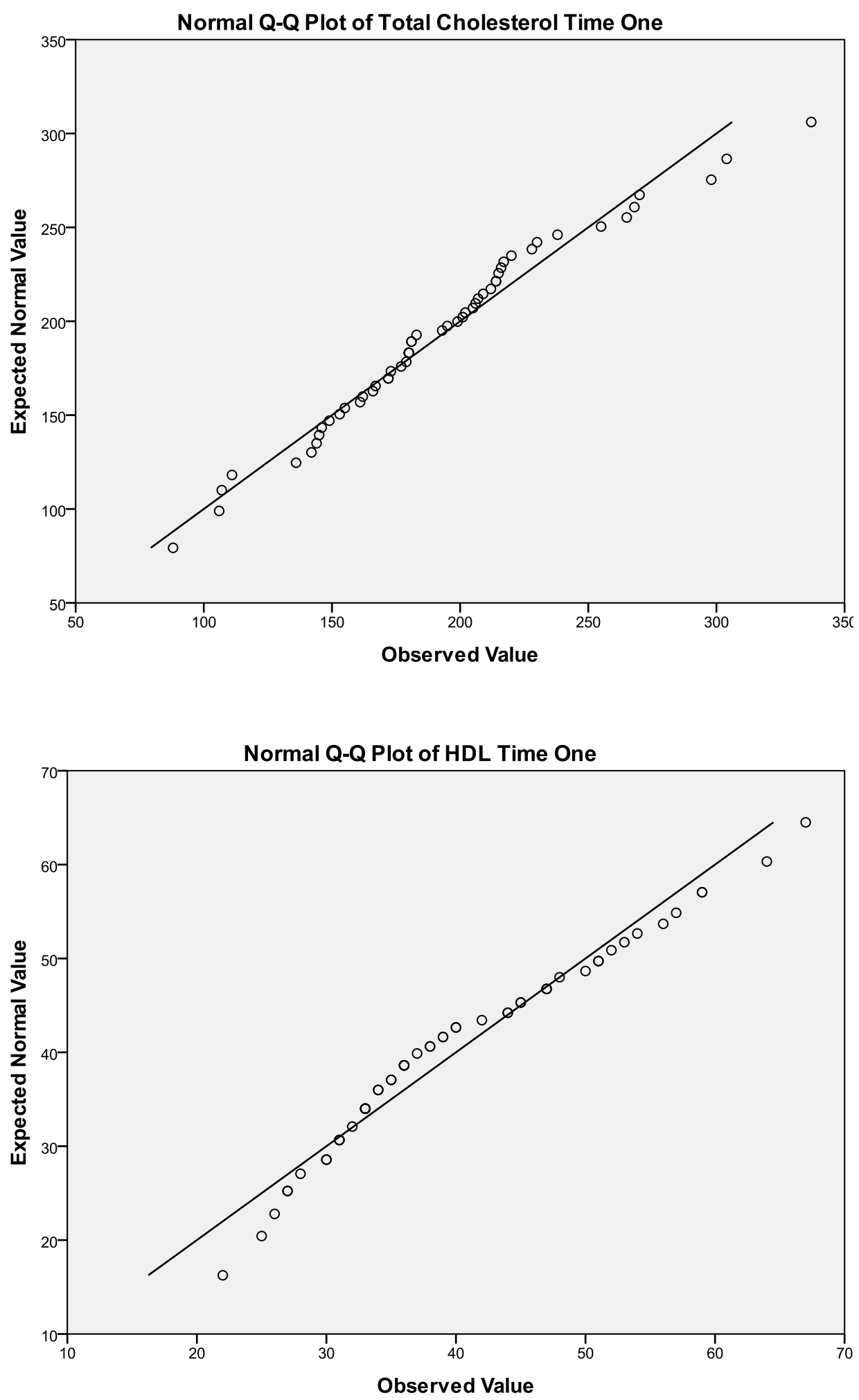

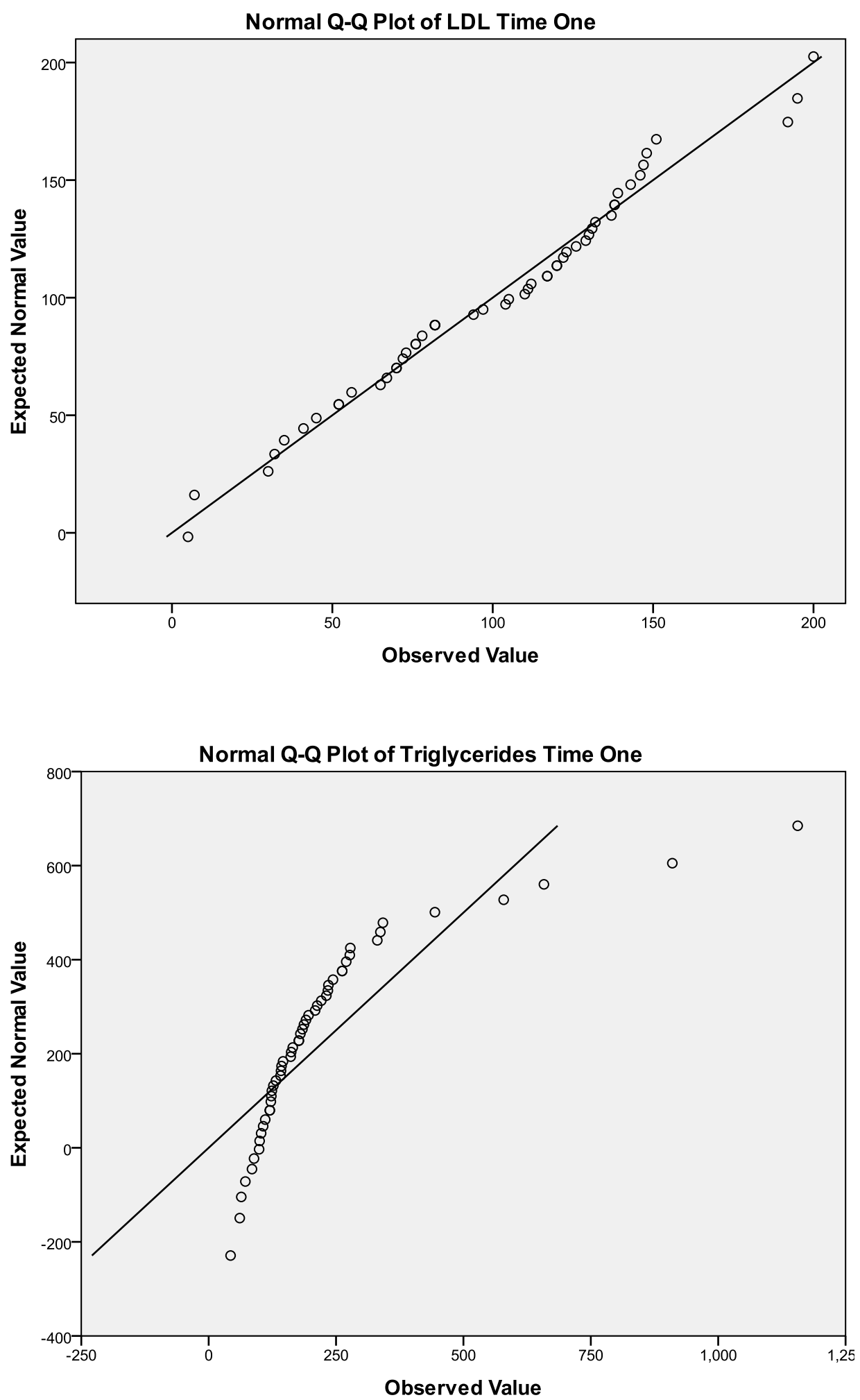

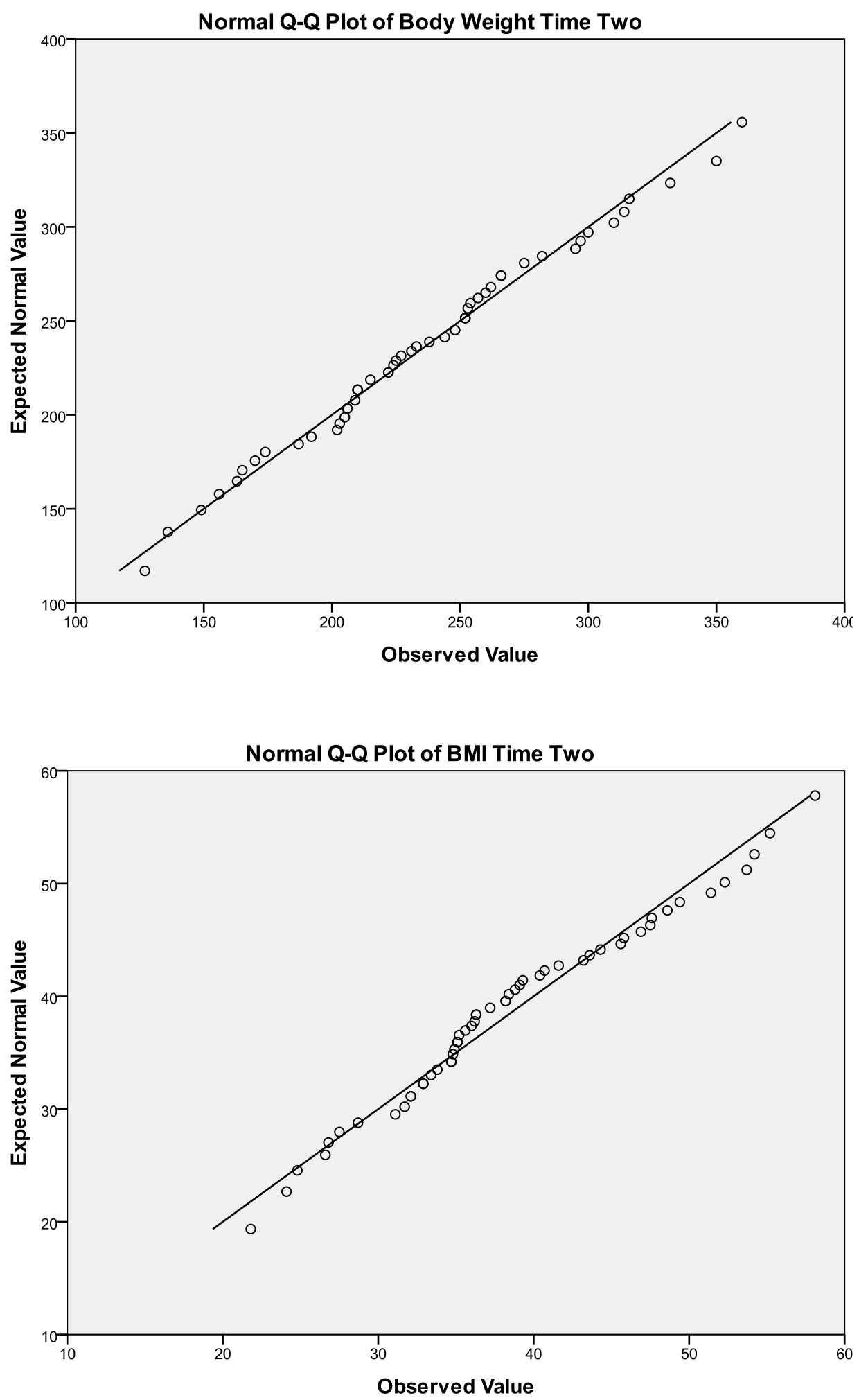

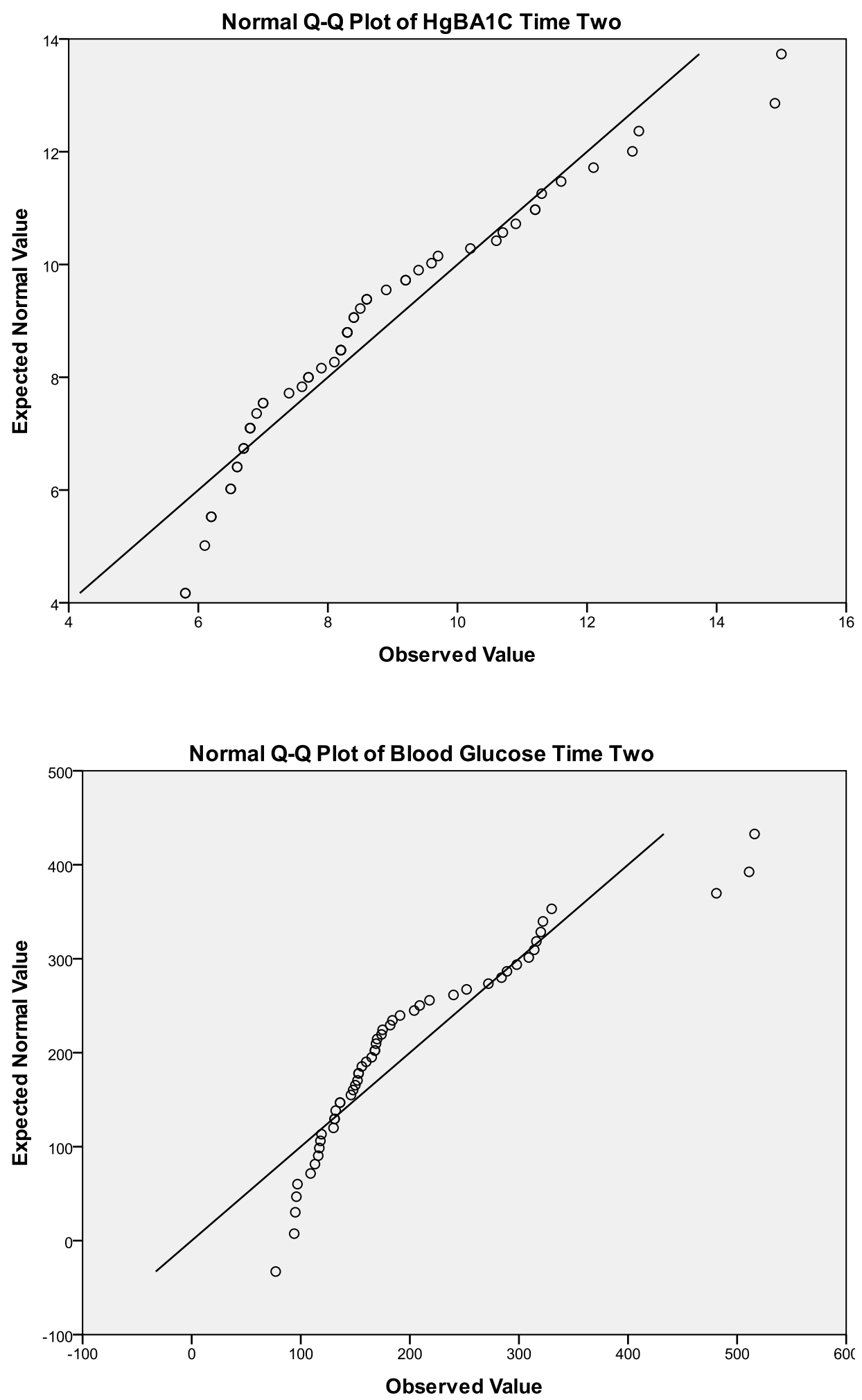

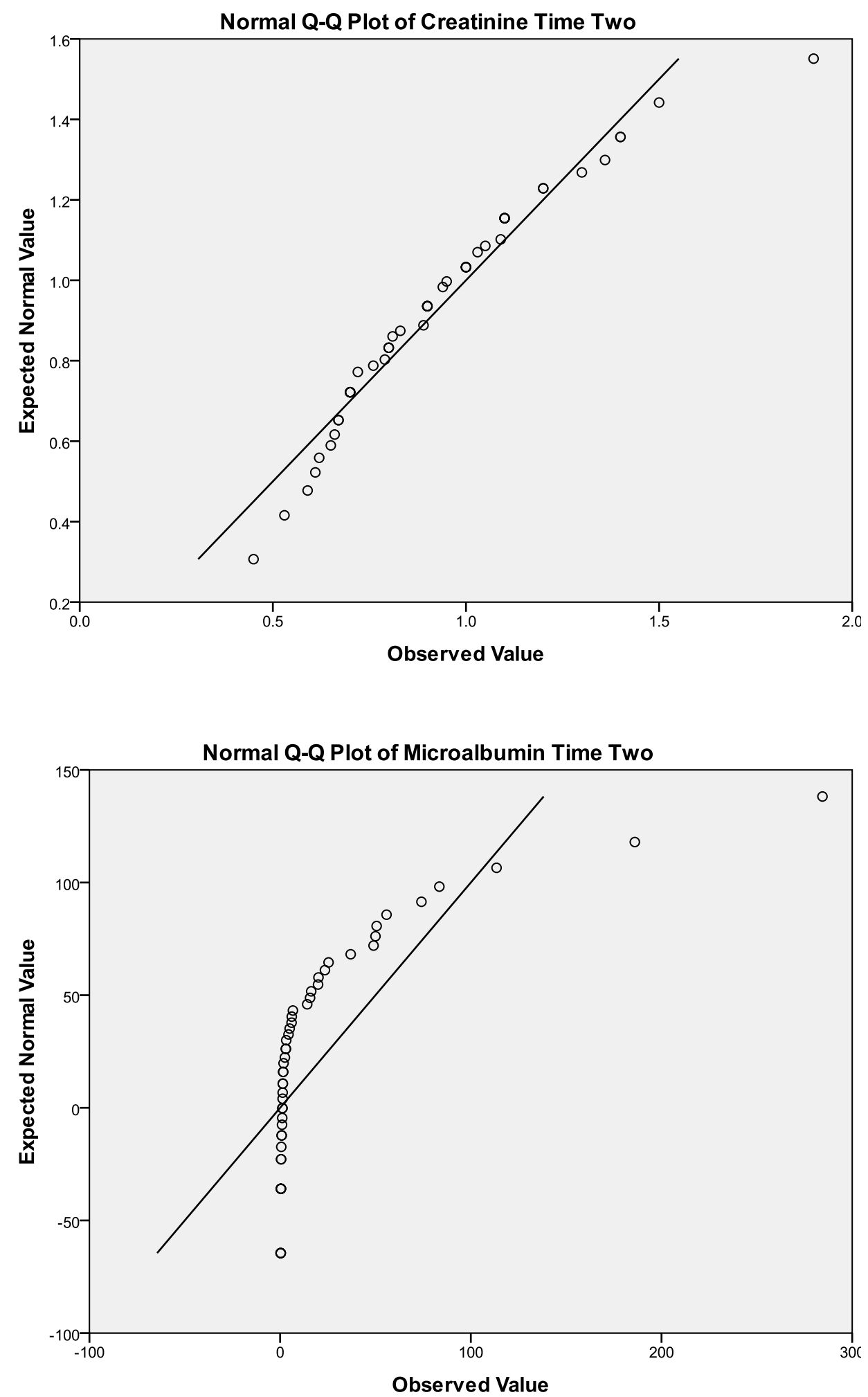

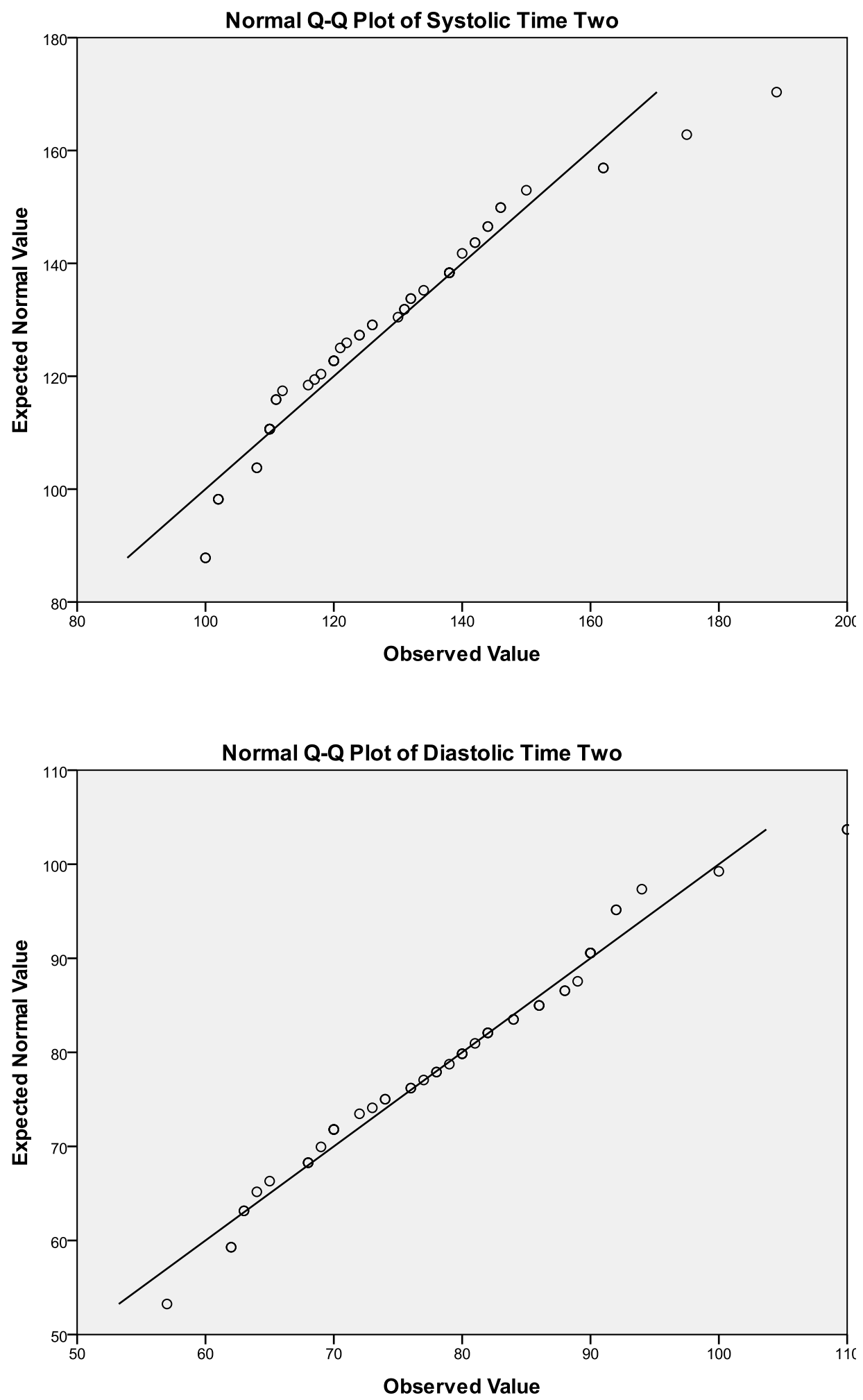

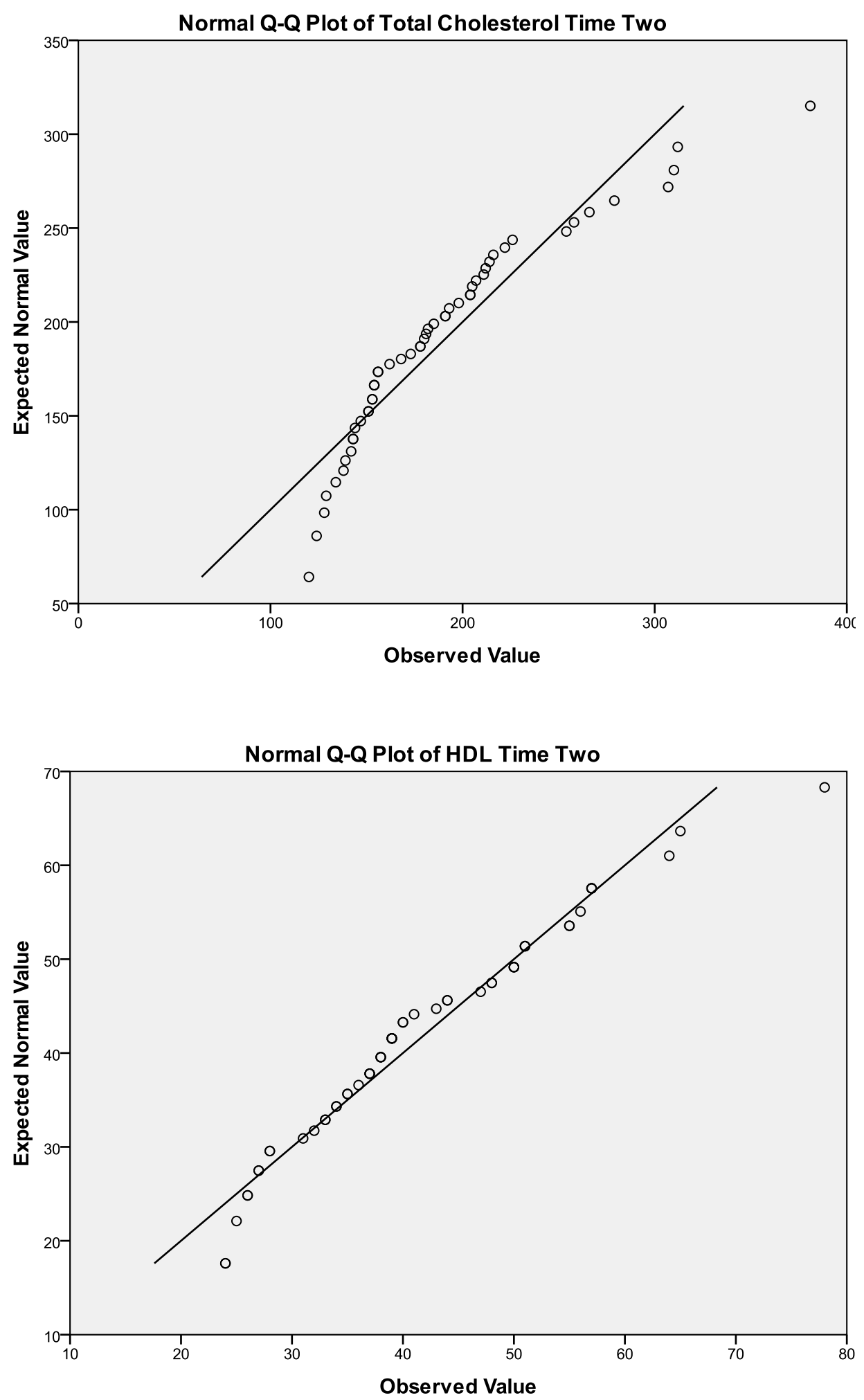

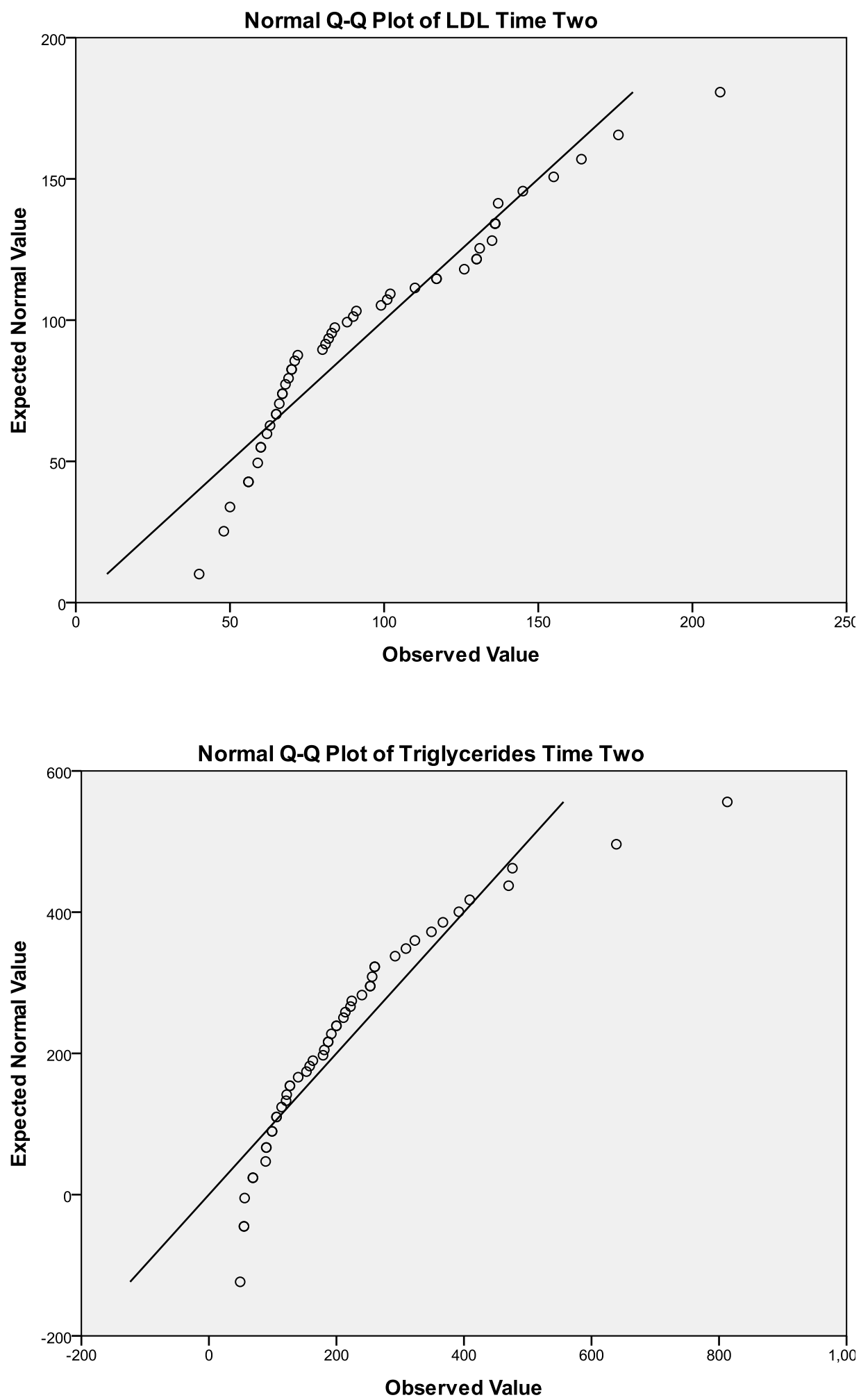
Appendix E: Q-Q Plots for Continuous Characteristics for Attendees of DGMVs
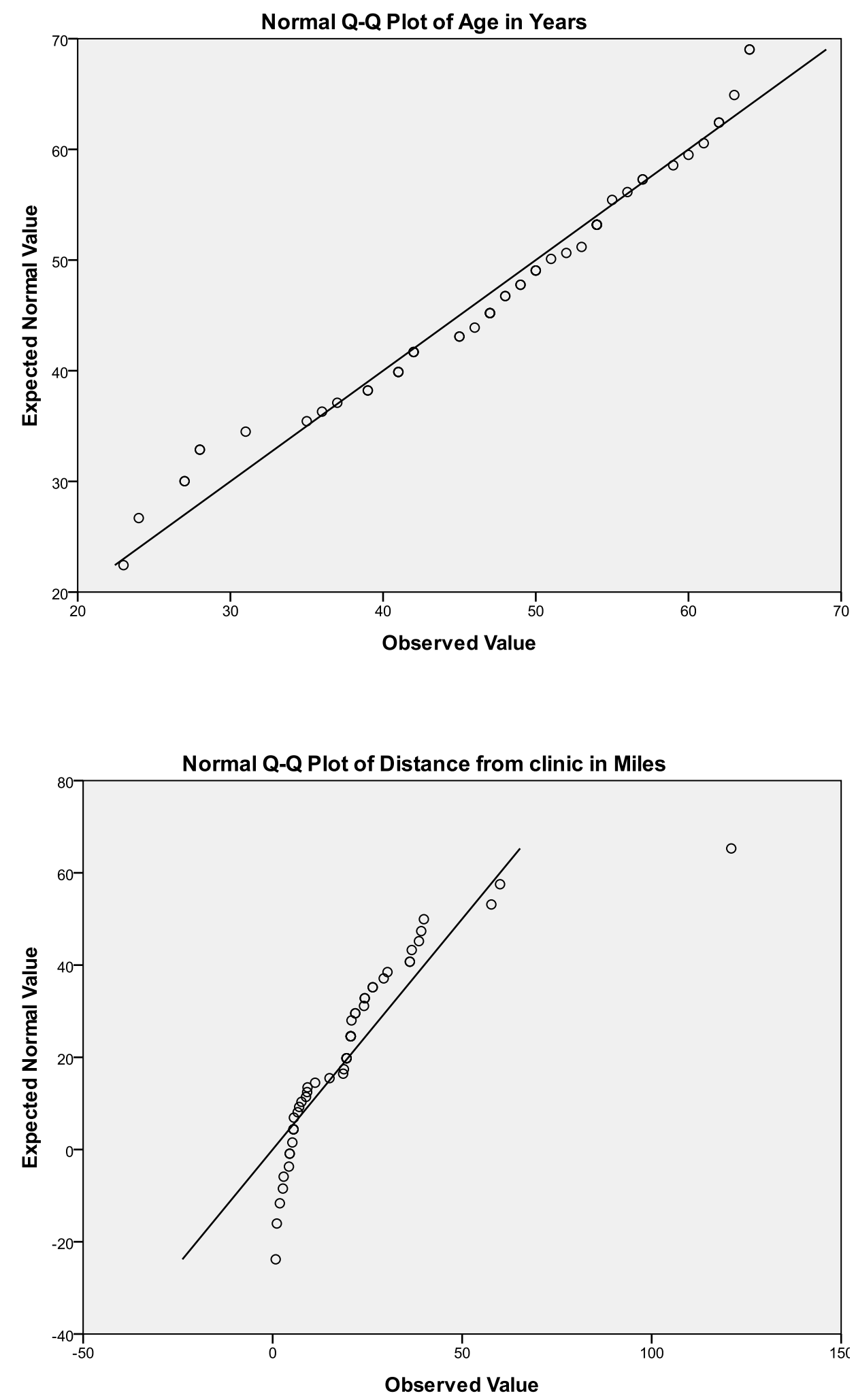


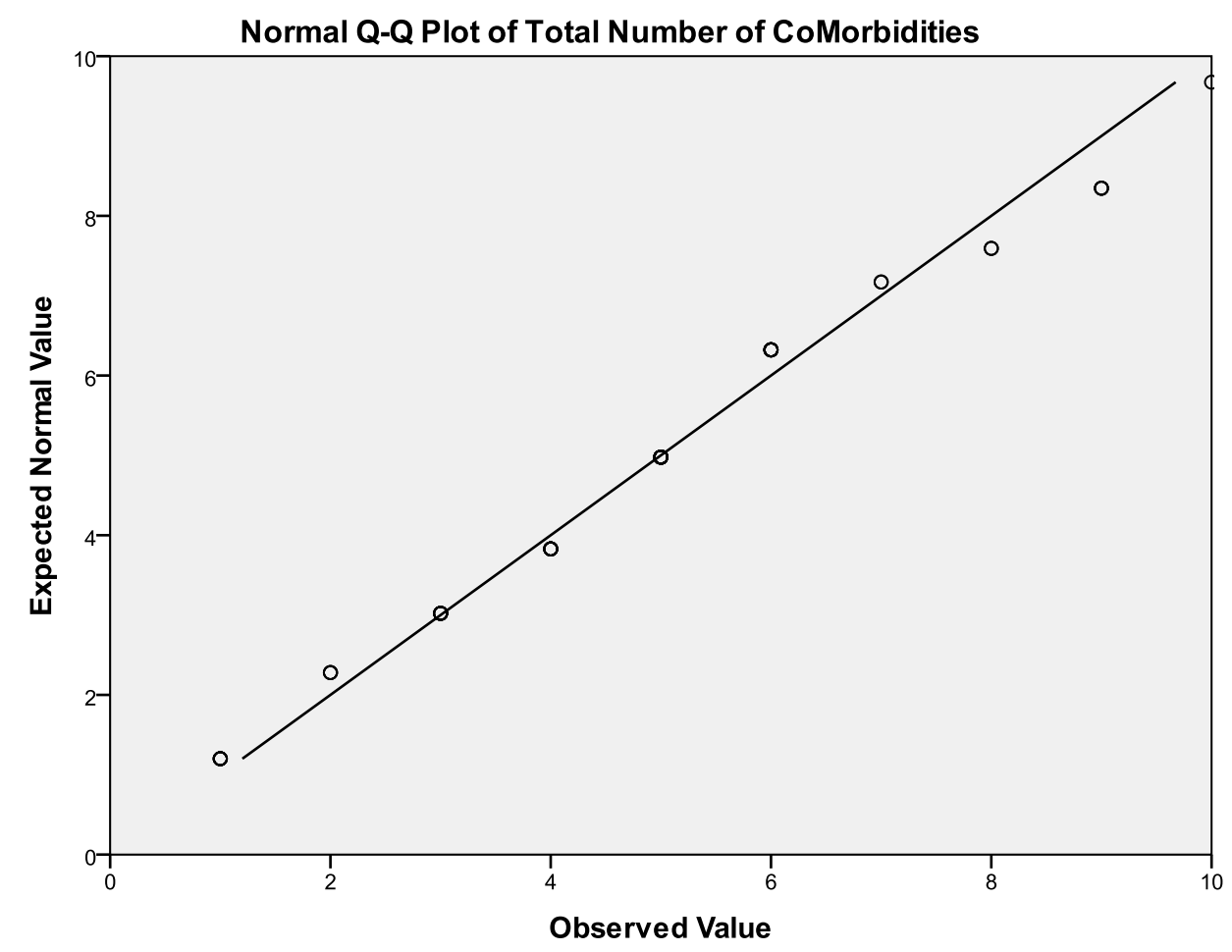




\section{Appendix F: Q-Q Plots for Continuous Characteristics for Usual Care}
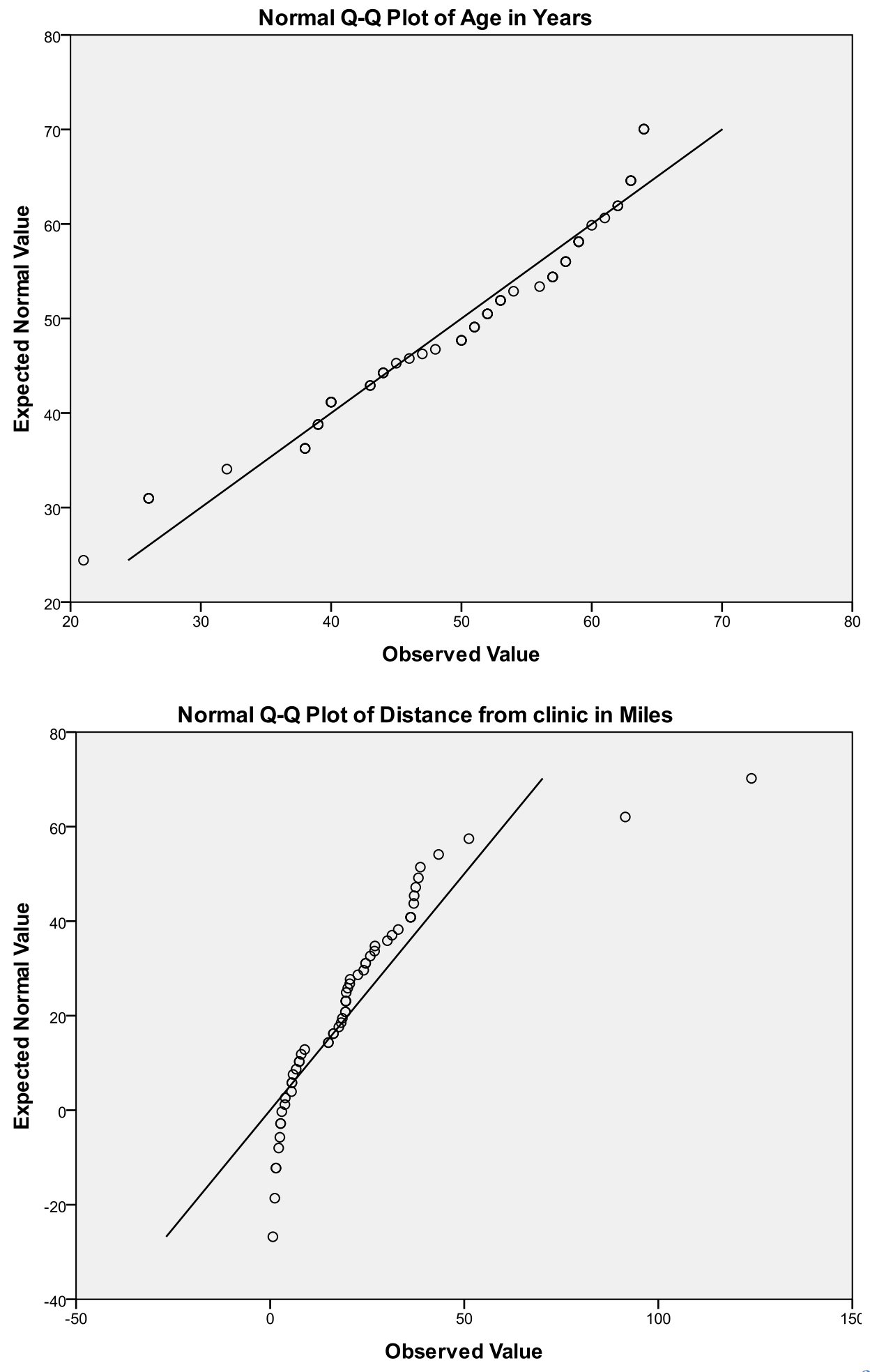


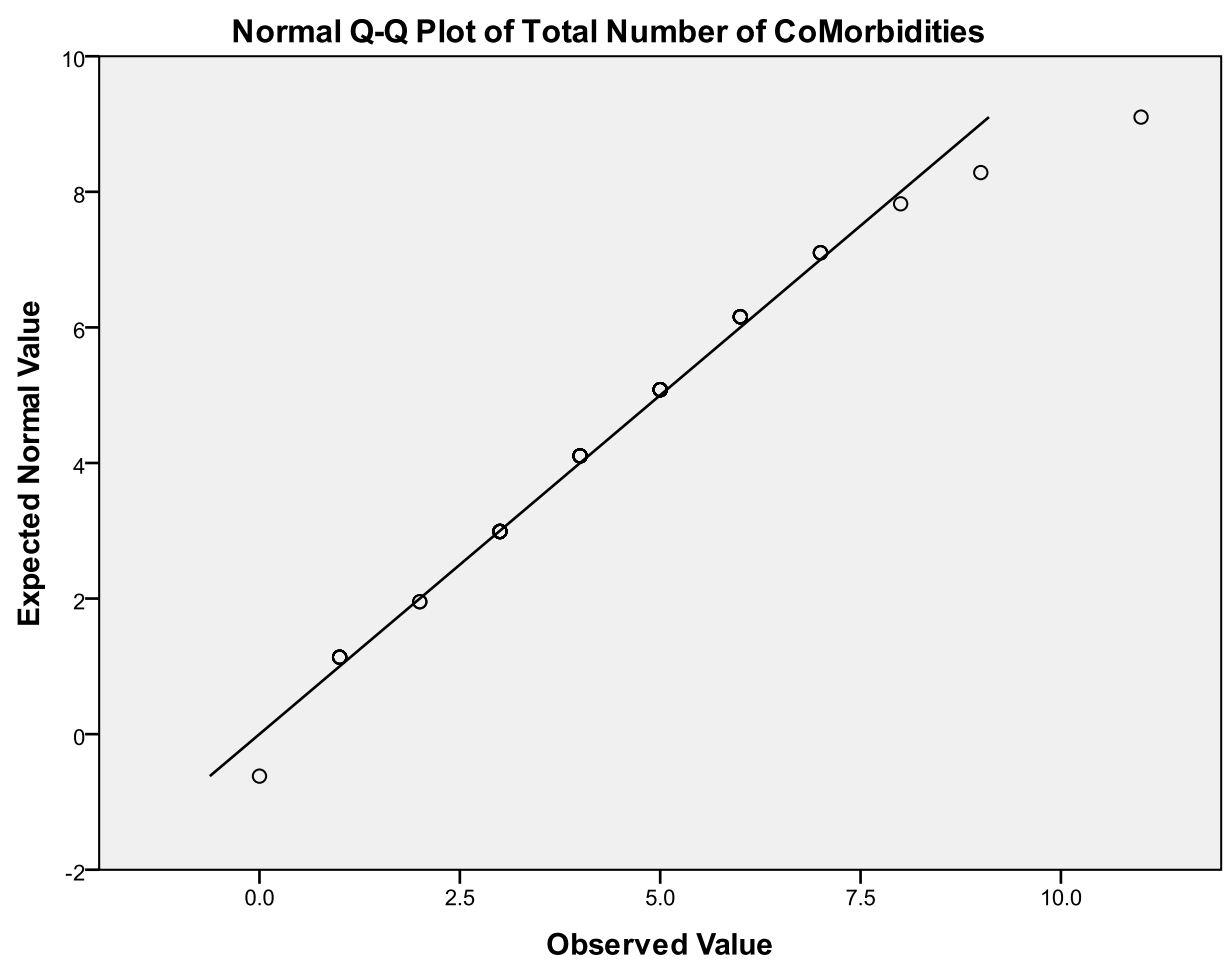

John $\mathrm{H}$.

Hagen

Digitally signed by John H. Hagen
DN: cn =John H. Hagen, o=West Virgin 